\title{
Recent results for the Landau-Lifshitz equation
}

\author{
André de Laire ${ }^{1}$
}

\begin{abstract}
We give a survey on some recent results concerning the Landau-Lifshitz equation, a fundamental nonlinear PDE with a strong geometric content, describing the dynamics of the magnetization in ferromagnetic materials. We revisit the Cauchy problem for the anisotropic LL equation, without dissipation, for smooth solutions, and also in the energy space in dimension one. We also examine two approximations of the LL equation given by of the Sine-Gordon equation and cubic Schrödinger equations, arising in certain singular limits of strong easy-plane and easy-axis anisotropy, respectively.

Concerning localized solutions, we review the orbital and asymptotic stability problems for a sum of solitons in dimension one, exploiting the variational nature of the solitons in the hydrodynamical framework.

Finally, we survey results concerning the existence, uniqueness and stability of self-similar solutions (expanders and shrinkers) for the isotropic LL equation with Gilbert term. Since expanders are associated with a singular initial condition with a jump discontinuity, we also review their well-posedness in spaces linked to the BMO space.
\end{abstract}

\section{Introduction}

The Landau-Lifshitz (LL) equation has been introduced in 1935 by L. Landau and E. Lifshitz in [76] and it constitutes nowadays a fundamental tool in the magnetic recording industry, due to its applications to ferromagnets [103]. This PDE describes the dynamics of the orientation of the magnetization (or spin) in ferromagnetic materials, and it is given by

$$
\partial_{t} \boldsymbol{m}+\boldsymbol{m} \times H_{\mathrm{eff}}(\boldsymbol{m})=0,
$$

where $\boldsymbol{m}=\left(m_{1}, m_{2}, m_{3}\right): \mathbb{R}^{N} \times I \longrightarrow \mathbb{S}^{2}$ is the spin vector, $I \subset \mathbb{R}$ is a time interval, $\times$ denotes the usual cross-product in $\mathbb{R}^{3}$, and $\mathbb{S}^{2}$ is the unit sphere in $\mathbb{R}^{3}$. Here $H_{\text {eff }}(\boldsymbol{m})$ is the effective magnetic field, corresponding to (minus) the $L^{2}$-derivative of the magnetic energy of the material. We will focus on energies of the form $E_{\mathrm{LL}}(\boldsymbol{m})=E_{\text {ex }}(\boldsymbol{m})+E_{\text {ani }}(\boldsymbol{m})$, where the exchange energy

$$
E_{\mathrm{ex}}(\boldsymbol{m})=\frac{1}{2} \int_{\mathbb{R}^{N}}|\nabla \boldsymbol{m}|^{2}=\frac{1}{2} \int_{\mathbb{R}^{N}}\left|\nabla m_{1}\right|^{2}+\left|\nabla m_{2}\right|^{2}+\left|\nabla m_{3}\right|^{2},
$$

accounts for the local tendency of $\boldsymbol{m}$ to align the magnetization field, and the anisotropy energy

$$
E_{\text {ani }}(\boldsymbol{m})=\frac{1}{2} \int_{\mathbb{R}^{N}}\langle\boldsymbol{m}, J \boldsymbol{m}\rangle_{\mathbb{R}^{3}}, \quad J \in \operatorname{Sym}_{3}(\mathbb{R}),
$$

accounts for the likelihood of $\boldsymbol{m}$ to attain one or more directions of magnetization, which determines the easy directions. Due to the invariance of (1) under rotations, we can assume that $J$ is a diagonal matrix $J=\operatorname{diag}\left(J_{1}, J_{2}, J_{3}\right)$, and thus the anisotropy energy reads

$$
E_{\text {ani }}(\boldsymbol{m})=\frac{1}{2} \int_{\mathbb{R}^{N}}\left(\lambda_{1} m_{1}^{2}+\lambda_{3} m_{3}^{2}\right),
$$

\footnotetext{
${ }^{1}$ Univ. Lille, CNRS, UMR 8524, Inria - Laboratoire Paul Painlevé, F-59000 Lille, France. E-mail: andre.de-laire@univ-lille.fr
} 
with $\lambda_{1}=J_{2}-J_{1}$ and $\lambda_{3}=J_{2}-J_{3}$. Therefore (1) can be recast as

$$
\partial_{t} \boldsymbol{m}+\boldsymbol{m} \times\left(\Delta \boldsymbol{m}-\lambda_{1} m_{1} \boldsymbol{e}_{1}-\lambda_{3} m_{3} \boldsymbol{e}_{3}\right)=0,
$$

where $\left(\boldsymbol{e}_{1}, \boldsymbol{e}_{2}, \boldsymbol{e}_{3}\right)$ is the canonical basis of $\mathbb{R}^{3}$. Notice that for finite energy solutions, 2 formally implies that $m_{1}(x) \rightarrow 0$ and $m_{3}(x) \rightarrow 0$, as $|x| \rightarrow \infty$, and hence $\left|m_{2}(x)\right| \rightarrow 1$, as $|x| \rightarrow \infty$.

For biaxial ferromagnets, all the numbers $J_{1}, J_{2}$ and $J_{3}$ are different, so that $\lambda_{1} \neq \lambda_{3}$ and $\lambda_{1} \lambda_{3} \neq 0$. Uniaxial ferromagnets are characterized by the property that only two of the numbers $J_{1}, J_{2}$ and $J_{3}$ are equal. For instance, the case $J_{1}=J_{2}$ corresponds to $\lambda_{1}=0$ and $\lambda_{3} \neq 0$, so that the material has a uniaxial anisotropy in the direction $\boldsymbol{e}_{3}$. Hence, the ferromagnet owns an easy-axis anisotropy along the vector $\boldsymbol{e}_{3}$ if $\lambda_{3}<0$, while the anisotropy is easy-plane along the plane $x_{3}=0$ if $\lambda_{3}>0$. Finally, in the isotropic case $\lambda_{1}=\lambda_{3}=0$, equation (3) reduces to the well-known Schrödinger map equation

$$
\partial_{t} \boldsymbol{m}+\boldsymbol{m} \times \Delta \boldsymbol{m}=0 .
$$

The LL equation (3) is a nonlinear dispersive PDE, with dispersion relation

$$
\omega(k)= \pm \sqrt{|k|^{4}+\left(\lambda_{1}+\lambda_{3}\right)|k|^{2}+\lambda_{1} \lambda_{3}},
$$

for linear sinusoidal waves of frequency $\omega$ and wavenumber $k$, i.e. solutions of the form $e^{i(k \cdot x-\omega t)}$. From (5), we can recognize similarities with some classical dispersive equations. For instance, for the Schrödinger equation $i \partial_{t} \psi+\Delta \psi=0$, the dispersion relation is $\omega(k)=|k|^{2}$, corresponding to $\lambda_{1}=\lambda_{3}=0$ in (5), i.e. the Schrödinger map equation (4).

When considering Schrödinger equations with nonvanishing conditions at infinity, the typical example is the Gross-Pitaesvkii equation 32.

$$
i \partial_{t} \psi+\Delta \psi+\sigma \psi\left(1-|\psi|^{2}\right)=0
$$

$\sigma>0$, and the dispersion relation for the linearized equation at the constant solution equal to 1 is $\omega(k)= \pm \sqrt{|k|^{4}+2 \sigma|k|^{2}}$. This corresponds to taking $\lambda_{1}=0$ or $\lambda_{3}=0$, with $\lambda_{1}+\lambda_{3}=2 \sigma$, in (5).

Finally, let us consider the Sine-Gordon equation $\partial_{t t} \psi-\Delta \psi+\sigma \sin (\psi)=0, \sigma>0$, whose linearized equation at 0 is given by the Klein-Gordon equation, with dispersion relation $\omega(k)=$ $\pm \sqrt{|k|^{2}+\sigma}$, that behaves like (5) for $\lambda_{1} \lambda_{3}=\sigma$ and $\lambda_{1}+\lambda_{3}=1$, at least for $k$ small.

In this context, the Landau-Lifshitz equation is considered as a universal model from which it is possible to derive other completely integrable equations [43. We review some recent rigorous results in this context in Section 3 .

\subsection{The dissipative model}

In 1955, T. Gilbert proposed in [53] a modification of equation (1) to incorporate a damping term. The so-called Landau-Lifshit-Gilbert (LLG) equation then reads

$$
\partial_{t} \boldsymbol{m}=-\beta \boldsymbol{m} \times H_{\mathrm{eff}}(\boldsymbol{m})-\alpha \boldsymbol{m} \times\left(\boldsymbol{m} \times H_{\mathrm{eff}}(\boldsymbol{m})\right),
$$

where $\beta \geq 0$ and $\alpha \geq 0$, so that there is dissipation when $\alpha>0$, and in that case we refer to $\alpha$ as the Gilbert damping coefficient. Note that, by performing a time scaling, we assume w.l.o.g. that

$$
\alpha \in[0,1] \text { and } \beta=\sqrt{1-\alpha^{2}} .
$$


Let us remark that the identity $a \times(b \times c)=b(a \cdot c)-c(a \cdot b)$, for all $a, b, c \in \mathbb{R}^{3}$, implies that for any smooth function $\boldsymbol{v}$, valued in $\mathbb{S}^{2}$, satisfies

$$
-\boldsymbol{v} \times(\boldsymbol{v} \times \Delta \boldsymbol{v})=\Delta \boldsymbol{v}+|\nabla \boldsymbol{v}|^{2} \boldsymbol{v} .
$$

Then, we see that in the limit case $\beta=0$ (and so $\alpha=1$ ), the LLG equation reduces to the heat-flow equation for harmonic maps

$$
\partial_{t} \boldsymbol{m}-\Delta \boldsymbol{m}=|\nabla \boldsymbol{m}|^{2} \boldsymbol{m} .
$$

This classical equation is an important model in several areas such as differential geometry and calculus of variations. It is also related with other problems such as the theory of liquid crystals and the Ginzburg-Landau equation. For more details, we refer to the surveys [41, 78, 96].

As before, one way to start the study of the LLG equation is noticing the link with other PDEs. Let us illustrate this point in the isotropic case $H_{\text {eff }}(\boldsymbol{m})=\Delta \boldsymbol{m}$. To simplify our notation, we consider the equation for the opposite vector $\boldsymbol{m} \rightarrow-\boldsymbol{m}$, which yields the equation

$$
\partial_{t} \boldsymbol{m}=\beta \boldsymbol{m} \times \Delta \boldsymbol{m}-\alpha \boldsymbol{m} \times(\boldsymbol{m} \times \Delta \boldsymbol{m}) .
$$

For a smooth solution $\boldsymbol{m}$ with $m_{3}>-1$, we can use the stereographic projection

$$
u=\mathcal{P}(\boldsymbol{m})=\frac{m_{1}+i m_{2}}{1+m_{3}},
$$

that satisfies the quasilinear Schrödinger equation

$$
i u_{t}+(\beta-i \alpha) \Delta u=2(\beta-i \alpha) \frac{\bar{u}(\nabla u)^{2}}{1+|u|^{2}},
$$

where we used the notation $(\nabla u)^{2}=\nabla u \cdot \nabla u=\sum_{j=1}^{N}\left(\partial_{x_{j}} u\right)^{2}$ (see e.g. [72] for details). When $\alpha>0$, one can use the properties of the semigroup $e^{(\alpha+i \beta) t \Delta}$ to establish a Cauchy theory for rough initial data, as we will see in Section 5 .

When $N=1$, the LLG equation is also related to the Localized Induction Approximation (LIA), also called binormal flow, a geometric curve flow modeling the self-induced motion of a vortex filament within an inviscid fluid in $\mathbb{R}^{3}$ [71, 31]. As we will in Section 5, this is related with the geometric representation of the LLG equation in a Serret-Frenet system.

There are several variants of previous equation considering more complex models including for instance a demagnetization field and the effects of the boundary in bounded domains. We refer to [71] for an overview of different models, to [29] for recent developments on the approximation of solutions, to the survey [57] for more details of the derivation and results on the initial value problem, and to [70] for a review of methods for pattern formation based on asymptotic analysis.

\subsection{The hydrodynamical formulation}

We end this introduction by explaining another useful transformation for the analysis of the LL equation. For simplicity, we assume that there is no dissipation. In the seminal work [80], Madelung showed that the nonlinear Schrödinger equation (NLS) can be recast into the form of a hydrodynamical system. For instance, for the NLS equation

$$
i \partial_{t} \Psi+\Delta \Psi+\Psi f\left(|\Psi|^{2}\right)=0,
$$


assuming that $\rho=|\Psi|^{2}$ does not vanish, the Madelung transform $\psi=\sqrt{\rho} e^{i \phi}$ leads to the system

$$
\partial_{t} \rho+2 \operatorname{div}(\rho \nabla \phi)=0, \quad \partial_{t} \phi+|\nabla \phi|^{2}+f(\rho)=\frac{\Delta(\sqrt{\rho})}{\sqrt{\rho}} .
$$

Therefore, setting $\boldsymbol{v}=2 \nabla \phi$, we get the Euler-Korteweg system

$$
\partial_{t} \rho+\operatorname{div}(\rho \boldsymbol{v})=0, \quad \partial_{t} \boldsymbol{v}+(\boldsymbol{v} \cdot \nabla) \boldsymbol{v}+2 \nabla(f(\rho))=2 \nabla\left(\frac{\Delta(\sqrt{\rho})}{\sqrt{\rho}}\right),
$$

which is a dispersive perturbation of the classical Euler equation for compressible fluids, with the additional term $2 \nabla(\Delta(\sqrt{\rho}) / \sqrt{\rho}$, which is interpreted as quantum pressure in the quantum fluids models [23, 20].

The Madelung transform is useful to study properties of NLS equations with nonvanishing conditions at infinity (see [14, 27). Coming back to the LL equation (3), let $\boldsymbol{m}$ be a solution of this equation such that the map $\check{m}=m_{1}+i m_{2}$ does not vanish. In the spirit of the Madelung transform, we set

$$
\check{m}=\left(1-m_{3}^{2}\right)^{\frac{1}{2}}(\sin (\phi)+i \cos (\phi)) .
$$

Thus, setting the hydrodynamical variables $u=m_{3}$ and $\phi$, we get the system

$$
\left\{\begin{array}{l}
\partial_{t} u=\operatorname{div}\left(\left(1-u^{2}\right) \nabla \phi\right)-\frac{\lambda_{1}}{2}\left(1-u^{2}\right) \sin (2 \phi), \\
\partial_{t} \phi=-\operatorname{div}\left(\frac{\nabla u}{1-u^{2}}\right)+u \frac{|\nabla u|^{2}}{\left(1-u^{2}\right)^{2}}-u|\nabla \phi|^{2}+u\left(\lambda_{3}-\lambda_{1} \sin ^{2}(\phi)\right),
\end{array}\right.
$$

at long as $|u|<1$ on $\mathbb{R}^{N}$. As shown in the next sections, the hydrodynamical formulation will be essential in the study of solutions of the LL equation.

Although it does not quite have the reputation of e.g. the Navier-Stokes equation or the Ricci flow equation, it can be said that the LL equation is among the most intriguing and challenging PDEs. The mathematical appeal relies on the combination of difficulties from nonlinear Schrödinger equations and geometric evolution equations. The aim of this note is to survey some recent results concerning the different aspects of the LL equation, as follows. In Section 2 we revisit the Cauchy problem for the anisotropic LL equation, without dissipation. Concerning smooth solutions, the approach follows a methodology for quasilinear hyperbolic systems based on a priori estimates by using new well-tailored higher order energies. We also tackle a subtle well-posedness problem in one space dimension in the energy space by invoking the hydrodynamical formulation.

Section 3 examines approximations of the Landau-Lifshitz equation by the Sine-Gordon equation and cubic Schrödinger equations arising in certain singular limits of large easy-plane and easy-axis anisotropy, respectively, providing quantitative convergence results.

In Section 4 we review the orbital and asymptotic stability problems for sum of solitons and multisolitons for the easy-plane (undamped) LL equation in dimension one. Stability problems of this kind are well-established in the context of dissipative evolution equations. Here the hamiltonian structure plays an essential role, that we exploit in the hydrodynamical framework. The essential idea is to exploit the variational structure given by the energy and momentum so that stability is essentially captured by spectral bounds for the hessian of the combined functional.

Finally, in Section 5 we consider the (isotropic) dissipative LLG equation. We focus mainly on the one-dimensional analysis of self-similar solutions: expanders and shrinkers evolving from or towards a singular time. We survey results concerning their existence and uniqueness by using a 
moving frame argument that allows us to obtain the asymptotics of the profiles. We also consider the question of stability of expanders that calls for a well-posedness result for solutions with rough initial data.

\section{The Cauchy problem for the LL equation}

Despite some serious efforts to establish a complete Cauchy theory for the LL equation, several issues remain unknown. In this section we will focus on the LL equation without damping, for which the Cauchy theory is even more delicate to handle. Even in the case where the problem is isotropic, i.e. the Schrödinger map equation, there are several unknown aspects. Moreover, it is not always possible to adapt results for Schrödinger map equation to include anisotropic perturbations.

The study of well-posedness in the presence of a damping term is different. Indeed, for the LLG equation, some techniques related to parabolic equations and for the heat-flow for harmonic maps (6) can be used. We will discuss this issue in Section 5 .

\subsection{The Cauchy problem for smooth solutions}

Let us consider the anisotropic LL equation (3) with $\lambda_{1}, \lambda_{3} \geq 0$. Since the associated energy is given by

$$
E_{\lambda_{1}, \lambda_{3}}(\boldsymbol{m})=\frac{1}{2} \int_{\mathbb{R}^{N}}\left(|\nabla \boldsymbol{m}|^{2}+\lambda_{1} m_{1}^{2}+\lambda_{3} m_{3}^{2}\right)
$$

the natural functional setting for solving this equation is the energy set

$$
\mathcal{E}_{\lambda_{1}, \lambda_{3}}\left(\mathbb{R}^{N}\right)=\left\{\boldsymbol{v} \in L_{\text {loc }}^{1}\left(\mathbb{R}^{N}, \mathbb{R}^{3}\right):|\boldsymbol{v}|=1 \text { a.e., } \nabla \boldsymbol{v} \in L^{2}\left(\mathbb{R}^{N}\right), \lambda_{1} v_{1}, \lambda_{3} v_{3} \in L^{2}\left(\mathbb{R}^{N}\right)\right\} .
$$

In the context of functions taking values on $\mathbb{S}^{2}$, it is standard to use the notation

$$
\mathcal{H}^{\ell}\left(\mathbb{R}^{N}\right)=\left\{\boldsymbol{v} \in L_{\text {loc }}^{1}\left(\mathbb{R}^{N}, \mathbb{R}^{3}\right):|\boldsymbol{v}|=1 \text { a.e., } \nabla \boldsymbol{v} \in H^{\ell-1}\left(\mathbb{R}^{N}\right)\right\},
$$

for an integer $\ell \geq 1$, where $H^{\ell-1}$ is the classical Sobolev space. Notice that a function $\boldsymbol{v} \in \mathcal{H}^{\ell}\left(\mathbb{R}^{N}\right)$ does not belong to $L^{2}\left(\mathbb{R}^{N}, \mathbb{R}^{3}\right)$, since this is incompatible with the constraint $|\boldsymbol{v}|=1$. In this manner, $\mathcal{E}_{\lambda_{1}, \lambda_{3}}\left(\mathbb{R}^{N}\right)$ reduces to $\mathcal{H}^{1}\left(\mathbb{R}^{N}\right)$ if $\lambda_{1}=\lambda_{3}=0$.

For the sake of simplicity, in this section we drop the subscripts $\lambda_{1}$ and $\lambda_{3}$, and denote the energy by $E(\boldsymbol{m})$ and the space by $\mathcal{E}\left(\mathbb{R}^{N}\right)$, since the constants $\lambda_{1}$ and $\lambda_{3}$ are fixed.

The first results concerning the existence of weak solutions of (3) in the energy space were obtained by Zhou and Guo in the one-dimensional case $N=1$ [104], and by Sulem, Sulem and Bardos [97] for $N \geq 1$. The approach followed in [104] was to consider a parabolic regularization by adding the term $\varepsilon \Delta \boldsymbol{m}$ and letting $\varepsilon \rightarrow 0$ (see e.g. [57]), while the strategy in [97] relied on finite difference approximations and a weak compactness argument. In both cases, no uniqueness was obtained. The proof in [97] can be generalized to include the anisotropic perturbation in (3), leading to the existence of a global (weak) solution as follows.

Theorem 2.1 ([97]). For any $\boldsymbol{m}_{0} \in \mathcal{E}\left(\mathbb{R}^{N}\right)$, there exists a global solution of $(3)$ with $\boldsymbol{m} \in$ $L^{\infty}\left(\mathbb{R}^{+}, \mathcal{E}\left(\mathbb{R}^{N}\right)\right)$, associated with the initial condition $\boldsymbol{m}_{0}$.

The uniqueness of the solution in Theorem 2.1 not known. To our knowledge, the wellposedness of the Landau-Lifshitz equation for general initial data in $\mathcal{E}\left(\mathbb{R}^{N}\right)$ remains an open question. 
Let us now discuss some results about smooth solutions in $\mathcal{H}^{k}\left(\mathbb{R}^{N}\right), k \in \mathbb{N}$, in the isotropic case $\lambda_{1}=\lambda_{3}=0$. For an initial data in $\boldsymbol{m}_{0} \in \mathcal{H}^{k}\left(\mathbb{R}^{N}\right)$, Sulem, Sulem and Bardos [97] proved the local existence and uniquenes $]^{1}$ of a solution $\boldsymbol{m} \in L^{\infty}\left([0, T), \mathcal{H}^{k}\left(\mathbb{R}^{N}\right)\right)$, provided that $k>N / 2+2$. By using a parabolic approximation, Ding and Wang [40] proved the local existence in $L^{\infty}\left([0, T), \mathcal{H}^{k}\left(\mathbb{R}^{N}\right)\right)$, provided that $k>N / 2+1$. They also study the difference between two solutions, obtaining uniqueness provided that the solutions are of class $\mathcal{C}^{3}$. Another approach was used by McMahagan [87, showing the existence as the limit of solutions of a perturbed wave problem, and using parallel transport to compare two solutions, to conclude local existence and uniqueness in $L^{\infty}\left([0, T), \mathcal{H}^{k}\left(\mathbb{R}^{N}\right)\right)$, for $k>N / 2+1$.

When $N=1$, these results provided the local existence and uniqueness at level $\mathcal{H}^{k}\left(\mathbb{R}^{N}\right)$, for $k \geq 2$. Moreover, in this case the solutions are global in time (see [91, 25]).

Of course, there is a large amount of other works with interesting results about the (local and global) existence and uniqueness for the LL equation and other related equations, see e.g. [9, 57, 58, 56, 65, 94] and the references therein. However, it is not straightforward to adapt these works to obtain local well-posednes results for smooth solutions to equation (3). For this reason, in the rest of this section we provide an alternative proof for local well-posedness by introducing high order energy quantities with better symmetrization properties.

To study the Cauchy problem of smooth solutions, given an integer $k \geq 1$, we introduce the set

$$
\mathcal{E}^{k}\left(\mathbb{R}^{N}\right)=\mathcal{E}\left(\mathbb{R}^{N}\right) \cap \mathcal{H}^{k}\left(\mathbb{R}^{N}\right)
$$

which we endow with the metric structure provided by the norm

$$
\|\boldsymbol{v}\|_{Z^{k}}=\left(\|\nabla \boldsymbol{v}\|_{H^{k-1}}^{2}+\left\|\boldsymbol{v}_{2}\right\|_{L^{\infty}}^{2}+\lambda_{1}\left\|\boldsymbol{v}_{1}\right\|_{L^{2}}^{2}+\lambda_{3}\left\|\boldsymbol{v}_{3}\right\|_{L^{2}}^{2}\right)^{\frac{1}{2}} .
$$

Observe that the energy space $\mathcal{E}\left(\mathbb{R}^{N}\right)$ identifies with $\mathcal{E}^{1}\left(\mathbb{R}^{N}\right)$. The uniform control on the second component $\boldsymbol{v}_{2}$ in the $Z^{k}$-norm ensures that $\|\cdot\|_{Z^{k}}$ is a norm. Of course, this uniform control is not the only possible choice of the metric structure. The main result of this section is the following local well-posedness result.

Theorem 2.2 ([35]). Let $\lambda_{1}, \lambda_{3} \geq 0$ and $k \in \mathbb{N}$, with $k>N / 2+1$. For any initial condition $\boldsymbol{m}^{0} \in \mathcal{E}^{k}\left(\mathbb{R}^{N}\right)$, there exist $T_{\max }>0$ and a unique solution $\boldsymbol{m}: \mathbb{R}^{N} \times\left[0, T_{\max }\right) \rightarrow \mathbb{S}^{2}$ to the $L L$ equation (3), which satisfies the following statements.

(i) The solution $\boldsymbol{m}$ belongs to $L^{\infty}\left([0, T], \mathcal{E}^{k}\left(\mathbb{R}^{N}\right)\right)$ and $\partial_{t} \boldsymbol{m} \in L^{\infty}\left([0, T], \mathcal{H}^{k-2}\left(\mathbb{R}^{N}\right)\right)$, for all $T \in\left(0, T_{\max }\right)$.

(ii) If the maximal time of existence $T_{\max }$ is finite, then

$$
\int_{0}^{T_{\max }}\|\nabla \boldsymbol{m}(t)\|_{L^{\infty}}^{2} d t=\infty .
$$

(iii) The flow map $\boldsymbol{m}^{0} \mapsto \boldsymbol{m}$ is well-defined and locally Lipschitz continuous from $\mathcal{E}^{k}\left(\mathbb{R}^{N}\right)$ to $\mathcal{C}^{0}\left([0, T], \mathcal{E}^{k-1}\left(\mathbb{R}^{N}\right)\right)$, for all $T \in\left(0, T_{\max }\right)$.

(iv) The energy (9) is conserved along the flow.

Theorem 2.2 provides the local well-posedness of the LL equation in the set $\mathcal{E}^{k}\left(\mathbb{R}^{N}\right)$. This kind of statement is standard in the context of hyperbolic systems (see e.g. [98, Theorem 1.2]). The critical regularity for the equation is given by the condition $k=N / 2$, so that local well-posedness is expected when $k>N / 2+1$. This assumption is used to control uniformly the gradient of the solutions by the Sobolev embedding theorem.

\footnotetext{
${ }^{1}$ Actually, in 97 they do not study of the difference between two solutions. It is only asserted that uniqueness followed from regularity, which it is not clear in this case; see also [65.
} 
The proof of Theorem 2.2 is based on energy estimates using well-tailored high order energies. A key observation is that any smooth function $\boldsymbol{m}$ valued into $\mathbb{S}^{2}$, satisfies the pointwise identities

$$
\left\langle\boldsymbol{m}, \partial_{i} \boldsymbol{m}\right\rangle_{\mathbb{R}^{3}}=\left\langle\boldsymbol{m}, \partial_{i i} \boldsymbol{m}\right\rangle_{\mathbb{R}^{3}}+\left|\partial_{i} \boldsymbol{m}\right|^{2}=\left\langle\boldsymbol{m}, \partial_{i i j} \boldsymbol{m}\right\rangle_{\mathbb{R}^{3}}+2\left\langle\partial_{i} \boldsymbol{m}, \partial_{i j} \boldsymbol{m}\right\rangle_{\mathbb{R}^{3}}+\left\langle\partial_{j} \boldsymbol{m}, \partial_{i i} \boldsymbol{m}\right\rangle_{\mathbb{R}^{3}}=0,
$$

for any $1 \leq i, j \leq N$. This allows us to show that a (smooth) solution to (3) satisfies the equation

$$
\partial_{t t} \boldsymbol{m}+\Delta^{2} \boldsymbol{m}-\left(\lambda_{1}+\lambda_{3}\right)\left(\Delta m_{1} \boldsymbol{e}_{1}+\Delta m_{3} \boldsymbol{e}_{3}\right)+\lambda_{1} \lambda_{3}\left(m_{1} \boldsymbol{e}_{1}+m_{3} \boldsymbol{e}_{3}\right)=F(\boldsymbol{m}),
$$

where we have set

$$
\begin{aligned}
F(\boldsymbol{m})= & \sum_{1 \leq i, j \leq N}\left(\partial_{i}\left(2\left\langle\partial_{i} \boldsymbol{m}, \partial_{j} \boldsymbol{m}\right\rangle_{\mathbb{R}^{3}} \partial_{j} \boldsymbol{m}-\left|\partial_{j} \boldsymbol{m}\right|^{2} \partial_{i} \boldsymbol{m}\right)-2 \partial_{i j}\left(\left\langle\partial_{i} \boldsymbol{m}, \partial_{j} \boldsymbol{m}\right\rangle_{\mathbb{R}^{3}} \boldsymbol{m}\right)\right) \\
& +\lambda_{1} F_{1,3}^{+}(\boldsymbol{m})+\lambda_{3} F_{3,1}^{-}(\boldsymbol{m})+\lambda_{1} \lambda_{3}\left(\left(m_{1}^{2}+m_{3}^{2}\right) \boldsymbol{m}+m_{1}^{2} m_{3} \boldsymbol{e}_{3}+m_{3}^{2} m_{1} \boldsymbol{e}_{1}\right)
\end{aligned}
$$

with

$$
\begin{aligned}
F_{i, j}^{ \pm}(\boldsymbol{m})= & \operatorname{div}\left(\left(m_{j}^{2}-2 m_{i}^{2}\right) \nabla \boldsymbol{m}+\left(m_{1} m_{3} \boldsymbol{e}_{3} \pm m_{1} \boldsymbol{m}-m_{3}^{2} \boldsymbol{e}_{1}\right) \nabla m_{1}+\left(m_{1} m_{3} \boldsymbol{e}_{1} \mp m_{3} \boldsymbol{m}-m_{1}^{2} \boldsymbol{e}_{3}\right) \nabla m_{3}\right) \\
& \pm \nabla m_{1} \cdot\left(m_{1} \nabla \boldsymbol{m}-\boldsymbol{m} \nabla m_{1}\right) \pm \nabla m_{3} \cdot\left(\boldsymbol{m} \nabla m_{3}-m_{3} \nabla \boldsymbol{m}\right)+m_{j}|\nabla \boldsymbol{m}|^{2} \boldsymbol{e}_{j} \\
& +\left(m_{1} \nabla m_{3}-m_{3} \nabla m_{1}\right) \cdot\left(\nabla m_{1} \boldsymbol{e}_{3}-\nabla m_{3} \boldsymbol{e}_{1}\right)+\lambda_{i} m_{i}^{2}\left(m_{i} \boldsymbol{e}_{i}-\boldsymbol{m}\right) .
\end{aligned}
$$

In view of (11), we define the (pseudo)energy of order $k \geq 2$, as

$$
E_{k}(t)=\left\|\partial_{t} \boldsymbol{m}\right\|_{\dot{H}^{k-2}}^{2}+\|\boldsymbol{m}\|_{\dot{H}^{k}}^{2}+\left(\lambda_{1}+\lambda_{3}\right)\left(\left\|m_{1}\right\|_{\dot{H}^{k-1}}^{2}+\left\|m_{3}\right\|_{\dot{H}^{k-1}}^{2}\right)+\lambda_{1} \lambda_{3}\left(\left\|m_{1}\right\|_{\dot{H}^{k-2}}^{2}+\left\|m_{3}\right\|_{\dot{H}^{k-2}}^{2}\right),
$$

for any $t \in[0, T]$. This high order energy is an anisotropic version of the one used in [97].

To get good energy estimates, we need to use Moser estimates (also called tame estimates) in Sobolev spaces (see e.g. [89]). Using these estimates and differentiating $E_{k}$, we obtain the following energy estimates.

Proposition 2.3. Let $\lambda_{1}, \lambda_{3} \geq 0$ and $k \in \mathbb{N}$, with $k>1+N / 2$. Assume that $\boldsymbol{m}$ is a solution to (3) in $\mathcal{C}^{0}\left([0, T], \mathcal{E}^{k+2}\left(\mathbb{R}^{N}\right)\right)$, with $\partial_{t} \boldsymbol{m} \in \mathcal{C}^{0}\left([0, T], H^{k}\left(\mathbb{R}^{N}\right)\right)$.

(i) The LL energy is well-defined and conserved along flow on $[0, T]$.

(ii) Given any integer $2 \leq \ell \leq k$, the energies $E_{\ell}$ are of class $\mathcal{C}^{1}$ on $[0, T]$, and there exists $C_{k}>0$, depending only on $k$, such that their derivatives satisfy

$$
E_{\ell}^{\prime}(t) \leq C_{k}\left(1+\left\|m_{1}(t)\right\|_{L^{\infty}}^{2}+\left\|m_{3}(t)\right\|_{L^{\infty}}^{2}+\|\nabla \boldsymbol{m}(t)\|_{L^{\infty}}^{2}\right) \Sigma_{\ell}(t),
$$

for any $t \in[0, T]$. Here, we have set $\Sigma_{\ell}=\sum_{j=1}^{\ell} E_{j}$.

We next discretize the equation by using a finite-difference scheme. The a priori bounds remain available in this discretized setting. We then apply standard weak compactness and local strong compactness results in order to construct local weak solutions, which satisfy statement (i) in Theorem 2.2. By applying the Gronwall lemma and the condition in (10), inequality (12) prevents a possible blow-up.

Finally, we establish uniqueness, as well as continuity with respect to the initial datum, by computing energy estimates for the difference of two solutions. More precisely, we show

Proposition 2.4. Let $\lambda_{1}, \lambda_{3} \geq 0$, and $k \in \mathbb{N}$, with $k>N / 2+1$. Consider two solutions $\boldsymbol{m}$ and $\tilde{\boldsymbol{m}}$ to $(3)$, which lie in $\mathcal{C}^{0}\left([0, T], \mathcal{E}^{k+1}\left(\mathbb{R}^{N}\right)\right)$, with $\partial_{t} \boldsymbol{m}, \partial_{t} \tilde{\boldsymbol{m}} \in \mathcal{C}^{0}\left([0, T], H^{k-1}\left(\mathbb{R}^{N}\right)\right)$, and set $\boldsymbol{u}=\tilde{\boldsymbol{m}}-\boldsymbol{m}$ and $\boldsymbol{v}=(\tilde{\boldsymbol{m}}+\boldsymbol{m}) / 2$. 
(i) The function $\mathfrak{E}_{0}(t)=\left\|\boldsymbol{u}(x, t)-u_{2}(x, 0) \boldsymbol{e}_{2}\right\|_{L^{2}}^{2}$ is of class $\mathcal{C}^{1}$ on $[0, T]$, and there exists $C>0$ such that for any $t \in[0, T]$,

$$
\begin{aligned}
\mathfrak{E}_{0}^{\prime}(t) \leq C(1+ & \|\nabla \tilde{\boldsymbol{m}}\|_{L^{2}}+\|\nabla \boldsymbol{m}(t)\|_{L^{2}}+\left\|\tilde{m}_{1}\right\|_{L^{2}}+\left\|m_{1}\right\|_{L^{2}} \\
& \left.+\left\|\tilde{m}_{3}\right\|_{L^{2}}+\left\|m_{3}\right\|_{L^{2}}\right)\left(\left\|\boldsymbol{u}-u_{2}^{0} e_{2}\right\|_{L^{2}}^{2}+\|\boldsymbol{u}\|_{L^{\infty}}^{2}+\|\nabla \boldsymbol{u}\|_{L^{2}}^{2}+\left\|\nabla u_{2}^{0}\right\|_{L^{2}}^{2}\right) .
\end{aligned}
$$

(ii) The function $\mathfrak{E}_{1}(t)=\|\nabla \boldsymbol{u}\|_{L^{2}}^{2}+\|\boldsymbol{u} \times \nabla \boldsymbol{v}+\boldsymbol{v} \times \nabla \boldsymbol{u}\|_{L^{2}}^{2}$ is of class $\mathcal{C}^{1}$ on $[0, T]$, and there exists $C>0$ such that

$$
\begin{aligned}
\mathfrak{E}_{1}^{\prime}(t) \leq C & \left(1+\|\nabla \boldsymbol{m}\|_{L^{\infty}}^{2}+\|\nabla \tilde{\boldsymbol{m}}\|_{L^{\infty}}^{2}\right)\left(\|\boldsymbol{u}\|_{L^{\infty}}^{2}+\|\nabla \boldsymbol{u}\|_{L^{2}}^{2}\right) \times \\
& \times\left(1+\|\nabla \boldsymbol{m}\|_{L^{\infty}}+\|\nabla \tilde{\boldsymbol{m}}\|_{L^{\infty}}+\|\nabla \boldsymbol{m}\|_{H^{1}}+\|\nabla \boldsymbol{m}\|_{H^{1}}\right) .
\end{aligned}
$$

(iii) Let $2 \leq \ell \leq k-1$,

$\mathfrak{E}_{\ell}(t)=\left\|\partial_{t} \boldsymbol{u}\right\|_{\dot{H}^{k-2}}^{2}+\|\boldsymbol{u}\|_{\dot{H}^{k}}^{2}+\left(\lambda_{1}+\lambda_{3}\right)\left(\left\|u_{1}\right\|_{\dot{H}^{k-1}}^{2}+\left\|u_{3}\right\|_{\dot{H}^{k-1}}^{2}\right)+\lambda_{1} \lambda_{3}\left(\left\|u_{1}\right\|_{\dot{H}^{k-2}}^{2}+\left\|u_{3}\right\|_{\dot{H}^{k-2}}^{2}\right)$,

and $\mathfrak{S}_{\mathrm{LL}}^{\ell}=\sum_{j=0}^{\ell} \mathfrak{E}_{\mathrm{LL}}^{j}$. Then $\mathfrak{E}_{\ell} \in \mathcal{C}^{1}([0, T])$, and there exists $C_{k}>0$, such that

$$
\begin{aligned}
\mathfrak{E}_{\ell}^{\prime}(t) \leq & C_{k}\left(1+\|\nabla \boldsymbol{m}\|_{H^{\ell}}^{2}+\|\nabla \tilde{\boldsymbol{m}}\|_{H^{\ell}}^{2}+\|\nabla \boldsymbol{m}\|_{L^{\infty}}^{2}+\|\nabla \tilde{\boldsymbol{m}}\|_{L^{\infty}}^{2}\right. \\
& \left.+\delta_{\ell=2}\left(\left\|\tilde{m}_{1}\right\|_{L^{2}}+\left\|m_{1}\right\|_{L^{2}}+\left\|\tilde{m}_{3}\right\|_{L^{2}}+\left\|m_{3}\right\|_{L^{2}}\right)\right)\left(\mathfrak{S}_{\mathrm{LL}}^{\ell}+\|\boldsymbol{u}\|_{L^{\infty}}^{2}\right) .
\end{aligned}
$$

When $\ell \geq 2$, the quantities $\mathfrak{E}_{\mathrm{LL}}^{\ell}$ in Proposition 2.4 are anisotropic versions of the ones used in [97. for similar purposes. Their explicit form is related to the linear part of the second-order equation in (11). The quantity $\mathfrak{E}_{\mathrm{LL}}^{0}$ is tailored to close off the estimates.

The introduction of the quantity $\mathfrak{E}_{\mathrm{LL}}^{1}$ is of a different nature. The functions $\nabla \boldsymbol{u}$ and $\boldsymbol{u} \times$ $\nabla \boldsymbol{v}+\boldsymbol{v} \times \nabla \boldsymbol{u}$ in its definition appear as the good variables to perform hyperbolic estimates at an $H^{1}$-level. They provide a better symmetrization corresponding to a further cancellation of the higher order terms. Without any use of the Hasimoto transform, nor of parallel transport, this makes possible a direct proof of local well-posedness at an $H^{k}$-level, with $k>N / 2+1$ instead of $k>N / 2+2$.

\subsection{Local well-posedness for smooth solutions}

To state a well-posedness result for $(\mathrm{H})$, we need to introduce a functional setting in which we can legitimate the use of the hydrodynamical framework. Under the condition $|\boldsymbol{m}|<1$, it is natural to work in the Hamiltonian framework in which the solutions $\boldsymbol{m}$ have finite energy. In the hydrodynamical formulation, the energy is given by

$$
E_{\mathrm{H}}(u, \varphi)=\frac{1}{2} \int_{\mathbb{R}^{N}}\left(\frac{|\nabla u|^{2}}{1-u^{2}}+\left(1-u^{2}\right)|\nabla \varphi|^{2}+\lambda_{1}\left(1-u^{2}\right) \sin ^{2}(\varphi)+\lambda_{3} u^{2}\right) .
$$

As a consequence, we work in the nonvanishing

$$
\mathcal{N} \mathcal{V}_{\sin }^{k}\left(\mathbb{R}^{N}\right)=\left\{(u, \varphi) \in H^{k}\left(\mathbb{R}^{N}\right) \times H_{\sin }^{k}\left(\mathbb{R}^{N}\right):|u|<1 \text { on } \mathbb{R}^{N}\right\}
$$

where

$$
H_{\text {sin }}^{k}\left(\mathbb{R}^{N}\right)=\left\{v \in L_{\text {loc }}^{1}\left(\mathbb{R}^{N}\right): \nabla v \in H^{k-1}\left(\mathbb{R}^{N}\right) \text { and } \sin (v) \in L^{2}\left(\mathbb{R}^{N}\right)\right\} .
$$

The set $H_{\sin }^{k}\left(\mathbb{R}^{N}\right)$ is an additive group, which is naturally endowed with the pseudometric distance

$$
d_{\sin }^{k}\left(v_{1}, v_{2}\right)=\left\|\sin \left(v_{1}-v_{2}\right)\right\|_{L^{2}}+\left\|\nabla v_{1}-\nabla v_{2}\right\|_{H^{k-1}},
$$


that vanishes if and only if $v_{1}-v_{2} \in \pi \mathbb{Z}$. This quantity is not a distance on the group $H_{\text {sin }}^{k}\left(\mathbb{R}^{N}\right)$, but it is on the quotient group $H_{\sin }^{k}\left(\mathbb{R}^{N}\right) / \pi \mathbb{Z}$. In the sequel, we identify the set $H_{\text {sin }}^{1}\left(\mathbb{R}^{N}\right)$ with this quotient group when necessary, in particular when a metric structure is required. This identification is not a difficulty as far as we deal with the hydrodynamical form of the LL equation and with the Sine-Gordon equation. Both the equations are indeed left invariant by adding a constant number in $\pi \mathbb{Z}$ to the phase functions. This property is one of the motivations for introducing the pseudometric distance $d_{\mathrm{sin}}^{k}$. We refer to [35] for more details concerning this distance, as well as the set $H_{\sin }^{k}\left(\mathbb{R}^{N}\right)$.

From Theorem 2.2, we obtain the following local well-posedness result for $(\mathrm{H})$.

Corollary $2.5([35])$. Let $\lambda_{1}, \lambda_{3} \geq 0$, and $k \in \mathbb{N}$, with $k>N / 2+1$. Given any $\left(u^{0}, \phi^{0}\right) \in$ $\mathcal{N} \mathcal{V}_{\sin }^{k}\left(\mathbb{R}^{N}\right)$, there exist $T_{\max }>0$ and a unique solution $(u, \phi): \mathbb{R}^{N} \times\left[0, T_{\max }\right) \rightarrow(-1,1) \times \mathbb{R}$ to $(\mathrm{H})$ with initial data $\left(u^{0}, \phi^{0}\right)$, which satisfies the following statements.

(i) The solution $(u, \phi)$ is in $L^{\infty}\left([0, T], \mathcal{N} \mathcal{V}_{\text {sin }}^{k}\left(\mathbb{R}^{N}\right)\right)$, while $\left(\partial_{t} u, \partial_{t} \phi\right)$ is in $L^{\infty}\left([0, T], H^{k-2}\left(\mathbb{R}^{N}\right)^{2}\right)$, for any $T \in\left(0, T_{\max }\right)$.

(ii) If the maximal time of existence $T_{\max }$ is finite, then

$$
\int_{0}^{T_{\max }}\left(\left\|\frac{\nabla u(t)}{\left(1-u(t)^{2}\right)^{\frac{1}{2}}}\right\|_{L^{\infty}}^{2}+\left\|\left(1-u(t)^{2}\right)^{\frac{1}{2}} \nabla \phi(t)\right\|_{L^{\infty}}^{2}\right) d t=\infty, \quad \text { or } \quad \lim _{t \rightarrow T_{\max }}\|u(t)\|_{L^{\infty}}=1 .
$$

(iii) The map $\left(u^{0}, \phi^{0}\right) \mapsto(u, \phi)$ is locally Lipschitz continuous from $\mathcal{N} \mathcal{V}_{\sin }^{k}\left(\mathbb{R}^{N}\right)$ to $\mathcal{C}^{0}\left([0, T], \mathcal{N} \mathcal{V}_{\sin }^{k-1}\left(\mathbb{R}^{N}\right)\right)$ for any $T \in\left(0, T_{\max }\right)$, and the energy $E_{\mathrm{H}}$ is conserved along the flow.

The proof of Corollary 2.5 is complicated by the metric structure corresponding to the set $H_{\text {sin }}^{k}\left(\mathbb{R}^{N}\right)$. Establishing the continuity of the flow map with respect to the pseudometric distance $d_{\mathrm{sin}}^{k}$ is not so immediate, but this difficulty can be by-passed by using some trigonometric identities.

\subsection{Local well-posedness in the energy space in dimension one}

We focus now on the LL equation with easy-plane anisotropy in dimension one, i.e. $\lambda_{1}=0$ and (3) reads

$$
\partial_{t} \boldsymbol{m}+\boldsymbol{m} \times\left(\partial_{x x} \boldsymbol{m}-\lambda_{3} m_{3} \boldsymbol{e}_{3}\right)=0 .
$$

As mentioned before, in the isotropic case $\lambda_{3}=0$, we have the local well-posedness for initial data in $\mathcal{H}^{2}(\mathbb{R})\left[25,90\right.$, 91]. Theorem 2.2 gives us for instance, the $\mathcal{H}^{2}$-local well-posedness, while Theorem 2.1 provides the existence of a solution in $\mathcal{H}^{1}(\mathbb{R})$, i.e. in the energy space for the isotropic equation. The isotropic equation is energy critical in $\mathcal{H}^{1 / 2}$, so that one could think that local well-posedness at the $\mathcal{H}^{1}$-level would be simple to establish. In this direction, when the domain is the torus, some progress has been made at the $H^{3 / 2^{+}}$-level [28], and an ill-posedness type result is given in 65] for the $H^{1 / 2}$-weak topology.

The purpose of this section is to provide a local well-posedness theory for 13 in the energy space, in the case $\lambda_{3} \geq 0$. To this end, we use the hydrodynamical version of the equation, considering hydrodynamical variables $u=m_{3}$ and $w=-\partial_{x} \varphi$, that is

$$
\left\{\begin{array}{l}
\partial_{t} u=\partial_{x}\left(\left(u^{2}-1\right) w\right), \\
\partial_{t} w=\partial_{x}\left(\frac{\partial_{x x} u}{1-u^{2}}+u \frac{\left(\partial_{x} u\right)^{2}}{\left(1-u^{2}\right)^{2}}+u\left(w^{2}-\lambda_{3}\right)\right) .
\end{array}\right.
$$


We introduce the notation $\mathfrak{u}=(u, w)$, that we will refer to as hydrodynamical pair. Notice that the LL energy is now expressed as

$$
E(\mathfrak{u})=\int_{\mathbb{R}} e(\mathfrak{u})=\frac{1}{2} \int_{\mathbb{R}}\left(\frac{\left(u^{\prime}\right)^{2}}{1-u^{2}}+\left(1-u^{2}\right) w^{2}+\lambda_{3} u^{2}\right),
$$

and the nonvanishing space is

$$
\mathcal{N} \mathcal{V}(\mathbb{R})=\left\{\mathfrak{v}=(v, w) \in H^{1}(\mathbb{R}) \times L^{2}(\mathbb{R}), \text { s.t. } \max _{\mathbb{R}}|v|<1\right\},
$$

endowed with the metric structure corresponding to the norm $\|\mathfrak{v}\|_{H^{1} \times L^{2}}=\|v\|_{H^{1}}+\|w\|_{L^{2}}$.

Another formally conserved quantity is the momentum $P$, which is defined by $P(\mathfrak{u})=\int_{\mathbb{R}} u w$. As we will see in Section 4, the momentum $P$, as well as the energy $E$, play an important role in the construction and the qualitative analysis of the solitons.

Concerning the Cauchy problem for $(\mathrm{H} 1 \mathrm{~d})$, we have the following local well-posedness result.

Theorem 2.6 ([34]). Let $\lambda_{3} \geq 0$ and $\mathfrak{u}^{0}=\left(u^{0}, w^{0}\right) \in \mathcal{N} \mathcal{V}(\mathbb{R})$. There exist $T_{\max }>0$ and $\mathfrak{u}=(u, w) \in \mathcal{C}^{0}\left(\left[0, T_{\max }\right), \mathcal{N} \mathcal{V}(\mathbb{R})\right)$, such that the following statements hold.

(i) The map $\mathfrak{u}$ is the unique solution to $(\overline{\mathrm{H} 1 \mathrm{~d}})$, with initial condition $\mathfrak{u}^{0}$, such that there exist smooth solutions $\mathfrak{u}_{n} \in \mathcal{C}^{\infty}(\mathbb{R} \times[0, T])$ to $(\overline{\mathrm{H} 1 \mathrm{~d}})$, which satisfy $\mathfrak{u}_{n} \rightarrow \mathfrak{u}$ in $\mathcal{C}^{0}([0, T], \mathcal{N} \mathcal{V}(\mathbb{R}))$, as $n \rightarrow \infty$, for any $T \in\left(0, T_{\max }\right)$. In addition, the energy $E$ and the momentum $P$ are constant on $\left(0, T_{\max }\right)$.

(ii) The maximal time $T_{\max }$ is characterized by the condition

$$
\lim _{t \rightarrow T_{\max }} \max _{x \in \mathbb{R}}|u(x, t)|=1, \quad \text { if } T_{\max }<\infty .
$$

(iii) When $\mathfrak{u}_{n}^{0} \rightarrow \mathfrak{u}^{0}$ in $H^{1}(\mathbb{R}) \times L^{2}(\mathbb{R})$, as $n \rightarrow \infty$, the maximal time of existence $T_{n}$ of the solution $\mathfrak{u}_{n}$ to $(\mathrm{H1d})$, with initial condition $\mathfrak{u}_{n}^{0}$, satisfies $T_{\max } \leq \lim \inf _{n \rightarrow \infty} T_{n}$, and $\mathfrak{u}_{n} \rightarrow \mathfrak{u}$ in $\mathcal{C}^{0}\left([0, T], H^{1}(\mathbb{R}) \times L^{2}(\mathbb{R})\right)$, as $n \rightarrow \infty$, for any $T \in\left(0, T_{\max }\right)$.

In other words, Theorem 2.6 provides the existence and uniqueness of a continuous flow for (H1d) in the energy space $\mathcal{N} \mathcal{V}(\mathbb{R})$. On the other hand, this does not prevent from the existence of other solutions which could not be approached by smooth solutions. In particular, we do not claim that there exists a unique local solution to $(\mathrm{H} 1 \mathrm{~d})$ in the energy space for a given initial condition. To our knowledge, the question of the global existence in the hydrodynamical framework of the local solution $\mathfrak{v}$ remains open. Concerning the equation (13), since we are in the one-dimensional case, it is possible to endow the energy space with the metric structure corresponding to the distance

$$
d_{\mathcal{E}}(\boldsymbol{u}, \boldsymbol{v})=\left(|\check{\boldsymbol{u}}(0)-\check{\boldsymbol{v}}(0)|^{2}+\left\|\boldsymbol{u}^{\prime}-\boldsymbol{v}^{\prime}\right\|_{L^{2}}^{2}+\lambda_{3}\left\|u_{3}-v_{3}\right\|_{L^{2}}^{2}\right)^{\frac{1}{2}}
$$

and to translate Theorem 2.6 into the original framework of the LL equation. This provides the existence of a unique continuous flow for $(13)$ in the neighborhood of solutions $\boldsymbol{m}$, such that the third component $\boldsymbol{m}_{3}$ does not reach the value \pm 1 . The flow is only locally defined due to this restriction.

The most difficult part in Theorem 2.6 is the continuity with respect to the initial data in the energy space $\mathcal{N} \mathcal{V}(\mathbb{R})$ when $\lambda_{3}>0$. In this case, by performing a change of variables, we can assume that $\lambda_{3}=1$. The proof relies on the strategy developed by Chang, Shatah and Uhlenbeck in [25] (see also [58, 90]), by introducing the map

$$
\Psi=\frac{1}{2}\left(\frac{\partial_{x} u}{\left(1-u^{2}\right)^{\frac{1}{2}}}+i\left(1-u^{2}\right)^{\frac{1}{2}} w\right) \exp i \theta, \quad \text { with } \theta(x, t)=-\int_{-\infty}^{x} u(y, t) w(y, t) d y .
$$


Then $\Psi$ solves the nonlinear Schrödinger equation

$$
i \partial_{t} \Psi+\partial_{x x} \Psi+2|\Psi|^{2} \Psi+\frac{1}{2} u^{2} \Psi-\operatorname{Re}(\Psi(1-2 F(u, \bar{\Psi})))(1-2 F(u, \Psi))=0,
$$

with $F(u, \Psi)(x, t)=\int_{-\infty}^{x} u(y, t) \Psi(y, t) d y$, while the function $u$ satisfies

$$
\partial_{t} u=2 \partial_{x} \operatorname{Im}(\Psi(2 F(u, \bar{\Psi})-1)), \quad \partial_{x} u=2 \operatorname{Re}(\Psi(1-2 F(u, \bar{\Psi}))) .
$$

In this setting, deriving the continuous dependence in $\mathcal{N} \mathcal{V}(\mathbb{R})$ of $\mathfrak{u}$ with respect to its initial data reduces to establish it for $u$ and $\Psi$ in $L^{2}(\mathbb{R})$. This can be done by combining an energy method for $u$ and classical Strichartz estimates for $\Psi$.

\section{Asymptotics regimes}

In this section we will study the connection between the LL equation

$$
\partial_{t} \boldsymbol{m}+\boldsymbol{m} \times\left(\Delta \boldsymbol{m}-\lambda_{1} m_{1} \boldsymbol{e}_{1}-\lambda_{3} m_{3} \boldsymbol{e}_{3}\right)=0,
$$

with $\lambda_{1}, \lambda_{3} \geq 0$, and the Sine-Gordon and the NLS equations, for certain types of anisotropies. More precisely, we investigate the cases when $\lambda_{1} \ll \lambda_{3}$ and when $1 \ll \lambda_{1}=\lambda_{3}$. A conjecture in the physical literature [93, 43] is that in the former case, the dynamics of (14) can be described by the Sine-Gordon equation, while in the latter case, can be approximated by the cubic NLS equation.

It is well-known that deriving asymptotic regimes is a powerful tool in order to tackle the analysis of intricate equations. In this direction, we expect that these rigorous derivations will be a useful tool to describe the dynamical properties of the LL equation, in particular the role played by the solitons in this dynamics. For instance, this kind of strategy has been useful in order to prove the asymptotic stability of the dark solitons of the Gross-Pitaevskii equation by using its link with the KdV equation (see [27, 13]).

\subsection{The Sine-Gordon regime}

In order to provide a rigorous mathematical statement for the anisotropic LL equation with $\lambda_{1} \ll \lambda_{3}$, i.e. for a strong easy-plane anisotropy regime, we consider a small parameter $\varepsilon>0$, a fixed constant $\sigma>0$, and set the anisotropy values $\lambda_{1}=\sigma \varepsilon$ and $\lambda_{3}=1 / \varepsilon$.

Assuming that the map $\check{m}=m_{1}+i m_{2}$, associated with a solution $\boldsymbol{m}$ to (14) does not vanish, we write $\check{m}=\left(1-m_{3}^{2}\right)^{\frac{1}{2}}(\sin (\phi)+i \cos (\phi))$, so that the variables $u=m_{3}$ and $\phi$ satisfy the system $(\mathrm{H})$, as long as the nonvanishing condition holds. To study the behavior of the system as $\varepsilon \rightarrow 0$, we introduce the rescaled variables $U_{\varepsilon}$ and $\Phi_{\varepsilon}$ given by

$$
U_{\varepsilon}(x, t)=\frac{u(x / \sqrt{\varepsilon}, t)}{\varepsilon}, \quad \text { and } \quad \Phi_{\varepsilon}(x, t)=\phi(x / \sqrt{\varepsilon}, t),
$$

which satisfy the hydrodynamical system

$$
\left\{\begin{array}{l}
\partial_{t} U_{\varepsilon}=\operatorname{div}\left(\left(1-\varepsilon^{2} U_{\varepsilon}^{2}\right) \nabla \Phi_{\varepsilon}\right)-\frac{\sigma}{2}\left(1-\varepsilon^{2} U_{\varepsilon}^{2}\right) \sin \left(2 \Phi_{\varepsilon}\right) \\
\partial_{t} \Phi_{\varepsilon}=U_{\varepsilon}\left(1-\varepsilon^{2} \sigma \sin ^{2}\left(\Phi_{\varepsilon}\right)\right)-\varepsilon^{2} \operatorname{div}\left(\frac{\nabla U_{\varepsilon}}{1-\varepsilon^{2} U_{\varepsilon}^{2}}\right)+\varepsilon^{4} U_{\varepsilon} \frac{\left|\nabla U_{\varepsilon}\right|^{2}}{\left(1-\varepsilon^{2} U_{\varepsilon}^{2}\right)^{2}}-\varepsilon^{2} U_{\varepsilon}\left|\nabla \Phi_{\varepsilon}\right|^{2} .
\end{array}\right.
$$


Therefore, as $\varepsilon \rightarrow 0$, we formally see that the limit system is

$$
\partial_{t} U=\Delta \Phi-\frac{\sigma}{2} \sin (2 \Phi), \quad \partial_{t} \Phi=U,
$$

so that the limit function $\Phi$ is a solution to the Sine-Gordon equation

$$
\partial_{t t} \Phi-\Delta \Phi+\frac{\sigma}{2} \sin (2 \Phi)=0
$$

As seen in Corollary 2.5 , the hydrodynamical system $\left(\mathrm{H}_{\varepsilon}\right)$ is locally well-posed in the space $\mathcal{N} \mathcal{V}_{\sin }^{k}\left(\mathbb{R}^{N}\right)$ for $k>N / 2+1$. However, this result gives us time of existence $T_{\varepsilon}$ that could vanish as $\varepsilon \rightarrow 0$. Therefore, we need to find a uniform estimate for $T_{\varepsilon}$ to prevent this phenomenon. As we will recall later, the Sine-Gordon equation is also locally well-posed at the same level of regularity, so that we can compare the evolution of the difference in an interval of time independent of $\varepsilon$. A further analysis of $\left(\overline{\mathrm{H}_{\varepsilon}}\right)$ involving good energy estimates, will lead us to the following result.

Theorem 3.1 ([35]). Let $N \geq 1$ and $k \in \mathbb{N}$, with $k>N / 2+1$, and $\varepsilon \in(0,1)$. Consider an initial condition $\left(U_{\varepsilon}^{0}, \Phi_{\varepsilon}^{0}\right) \in \mathcal{N} \overline{\mathcal{V}}_{\sin }^{k+2}\left(\mathbb{R}^{N}\right)$, and set

$$
\mathcal{K}_{\varepsilon}=\left\|U_{\varepsilon}^{0}\right\|_{H^{k}}+\varepsilon\left\|\nabla U_{\varepsilon}^{0}\right\|_{H^{k}}+\left\|\nabla \Phi_{\varepsilon}^{0}\right\|_{H^{k}}+\left\|\sin \left(\Phi_{\varepsilon}^{0}\right)\right\|_{H^{k}} .
$$

Consider similarly an initial condition $\left(U^{0}, \Phi^{0}\right) \in L^{2}\left(\mathbb{R}^{N}\right) \times H_{\sin }^{1}\left(\mathbb{R}^{N}\right)$, and denote by $(U, \Phi) \in$ $\mathcal{C}^{0}\left(\mathbb{R}, L^{2}\left(\mathbb{R}^{N}\right) \times H_{\sin }^{1}\left(\mathbb{R}^{N}\right)\right)$ the unique corresponding solution to (SGS). Then, there exists $C>0$, depending only on $\sigma, k$ and $N$, such that, if

$$
C \varepsilon \mathcal{K}_{\varepsilon} \leq 1,
$$

then the following statements hold.

(i) There exists a positive number $T_{\varepsilon} \geq\left(C \mathcal{K}_{\varepsilon}^{2}\right)^{-1}$, such that there is a unique solution $\left(U_{\varepsilon}, \Phi_{\varepsilon}\right) \in$ $\mathcal{C}^{0}\left(\left[0, T_{\varepsilon}\right], \mathcal{N} \mathcal{V}_{\sin }^{k+1}\left(\mathbb{R}^{N}\right)\right)$ to $\left(\mathrm{H}_{\varepsilon}\right)$ with initial data $\left(U_{\varepsilon}^{0}, \Phi_{\varepsilon}^{0}\right)$.

(ii) If $\Phi_{\varepsilon}^{0}-\Phi^{0} \in L^{2}\left(\mathbb{R}^{N}\right)$, then, for any $0 \leq t \leq T_{\varepsilon}$,

$$
\left\|\Phi_{\varepsilon}(t)-\Phi(t)\right\|_{L^{2}} \leq C\left(\left\|\Phi_{\varepsilon}^{0}-\Phi^{0}\right\|_{L^{2}}+\left\|U_{\varepsilon}^{0}-U^{0}\right\|_{L^{2}}+\varepsilon^{2} \mathcal{K}_{\varepsilon}\left(1+\mathcal{K}_{\varepsilon}^{3}\right)\right) e^{C t} .
$$

(iii) If $N \geq 2$, or $N=1$ and $k>N / 2+2$, then we have, for any $0 \leq t \leq T_{\varepsilon}$,

$$
\left\|U_{\varepsilon}(t)-U(t)\right\|_{L^{2}}+d_{\sin }^{1}\left(\Phi_{\varepsilon}(t), \Phi(t)\right) \leq C\left(\left\|U_{\varepsilon}^{0}-U^{0}\right\|_{L^{2}}+d_{\sin }^{1}\left(\Phi_{\varepsilon}^{0}, \Phi^{0}\right)+\varepsilon^{2} \mathcal{K}_{\varepsilon}\left(1+\mathcal{K}_{\varepsilon}^{3}\right)\right) e^{C t} .
$$

(iv) Let $\left(U^{0}, \Phi^{0}\right) \in H^{k}\left(\mathbb{R}^{N}\right) \times H_{\text {sin }}^{k+1}\left(\mathbb{R}^{N}\right)$ and set $\kappa_{\varepsilon}=\mathcal{K}_{\varepsilon}+\left\|U^{0}\right\|_{H^{k}}+\left\|\nabla \Phi^{0}\right\|_{H^{k}}+\left\|\sin \left(\Phi^{0}\right)\right\|_{H^{k}}$. There exists $A>0$, depending only on $\sigma, k$ and $N$, such that the solution $(U, \Phi)$ lies in $\mathcal{C}^{0}\left(\left[0, T_{\varepsilon}^{*}\right], H^{k}\left(\mathbb{R}^{N}\right) \times H_{\sin }^{k+1}\left(\mathbb{R}^{N}\right)\right)$, for some $T_{\varepsilon}^{*} \in\left[\frac{1}{A \kappa_{\varepsilon}^{2}}, T_{\varepsilon}\right]$. Moreover, when $k>N / 2+3$, we have, for any $0 \leq t \leq T_{\varepsilon}^{*}$,

$$
\begin{aligned}
& \left\|U_{\varepsilon}(t)-U(t)\right\|_{H^{k-3}}+\left\|\nabla \Phi_{\varepsilon}(t)-\nabla \Phi(t)\right\|_{H^{k-3}}+\left\|\sin \left(\Phi_{\varepsilon}(t)-\Phi(t)\right)\right\|_{H^{k-3}} \\
& \leq A e^{A\left(1+\kappa_{\varepsilon}^{2}\right) t}\left(\left\|U_{\varepsilon}^{0}-U^{0}\right\|_{H^{k-3}}+\left\|\nabla \Phi_{\varepsilon}^{0}-\nabla \Phi^{0}\right\|_{H^{k-3}}+\left\|\sin \left(\Phi_{\varepsilon}^{0}-\Phi^{0}\right)\right\|_{H^{k-3}}+\varepsilon^{2} \kappa_{\varepsilon}\left(1+\kappa_{\varepsilon}^{3}\right)\right) .
\end{aligned}
$$

In arbitrary dimension, Theorem 3.1 provides a quantified convergence of the LL equation towards the Sine-Gordon equation in the regime of strong easy-plane anisotropy. Three types of convergence are proved depending on the dimension, and the levels of regularity of the solutions. This trichotomy is related to the analysis of the Cauchy problems for the LL and Sine-Gordon equations. 
In its natural Hamiltonian framework, the Sine-Gordon equation is globally well-posed and its Hamiltonian is the Sine-Gordon energy:

$$
E_{\mathrm{SG}}(\phi)=\frac{1}{2} \int_{\mathbb{R}^{N}}\left(\left(\partial_{t} \phi\right)^{2}+|\nabla \phi|^{2}+\sigma \sin (\phi)^{2}\right) .
$$

More precisely, given an initial condition $\left(\Phi^{0}, \Phi^{1}\right) \in H_{\sin }^{1}\left(\mathbb{R}^{N}\right) \times L^{2}\left(\mathbb{R}^{N}\right)$, there exists a unique corresponding solution $\Phi \in \mathcal{C}^{0}\left(\mathbb{R}, H_{\sin }^{1}\left(\mathbb{R}^{N}\right)\right)$ to (SG), with $\partial_{t} \Phi \in \mathcal{C}^{0}\left(\mathbb{R}, L^{2}\left(\mathbb{R}^{N}\right)\right)$. Moreover, the Sine-Gordon equation is locally well-posed in the spaces $H_{\text {sin }}^{k}\left(\mathbb{R}^{N}\right) \times H^{k-1}\left(\mathbb{R}^{N}\right)$, when $k>N / 2+1$. In other words, the solution $\Phi$ remains in $\mathcal{C}^{0}\left([0, T], H_{\sin }^{k}\left(\mathbb{R}^{N}\right)\right)$, with $\partial_{t} \Phi \in \mathcal{C}^{0}\left([0, T], H^{k-1}\left(\mathbb{R}^{N}\right)\right)$, at least locally in time, when $\left(\Phi^{0}, \Phi^{1}\right) \in H_{\sin }^{k}\left(\mathbb{R}^{N}\right) \times H^{k-1}\left(\mathbb{R}^{N}\right)$. We refer to [35, 22] for more details about the Cauchy problem for (SG).

As seen in Section 2, the LL equation is locally well-posed at the same level of high regularity as the Sine-Gordon equation. In the hydrodynamical context, this reads as the existence of a maximal time $T_{\max }$ and a unique solution $(U, \Phi) \in \mathcal{C}^{0}\left(\left[0, T_{\max }\right), \mathcal{N} \mathcal{V}_{\sin }^{k-1}\left(\mathbb{R}^{N}\right)\right)$ to $\left(\mathrm{H}_{\varepsilon}\right)$ corresponding to an initial condition $\left(U^{0}, \Phi^{0}\right) \in \mathcal{N} \mathcal{V}_{\sin }^{k}\left(\mathbb{R}^{N}\right)$, when $k>N / 2+1$ (see Corollary 2.5); note the loss of one derivative here. This loss explains why we take initial conditions $\left(U_{\varepsilon}^{0}, \Phi_{\varepsilon}^{0}\right)$ in $\mathcal{N} \mathcal{V}_{\sin }^{k+2}\left(\mathbb{R}^{N}\right)$, though the quantity $\mathcal{K}_{\varepsilon}$ is already well-defined when $\left(U_{\varepsilon}^{0}, \Phi_{\varepsilon}^{0}\right) \in \mathcal{N} \mathcal{V}_{\sin }^{k+1}\left(\mathbb{R}^{N}\right)$.

In view of this local well-posedness result, we restrict our analysis of the Sine-Gordon regime to the solutions $\left(U_{\varepsilon}, \Phi_{\varepsilon}\right)$ to the rescaled system $\left(\mathrm{H}_{\varepsilon}\right)$ with sufficient regularity. A further difficulty then lies in the fact that their maximal times of existence possibly depend on $\varepsilon$.

Statement (i) in Theorem 3.1 provides an explicit control on these maximal times. Since $T_{\varepsilon} \geq\left(C \mathcal{K}_{\varepsilon}^{2}\right)^{-1}$, these maximal times are bounded from below by a positive number depending only on the choice of the initial data $\left(U_{\varepsilon}^{0}, \Phi_{\varepsilon}^{0}\right)$. Notice in particular that if a family of initial data $\left(U_{\varepsilon}^{0}, \Phi_{\varepsilon}^{0}\right)$ converges towards a pair $\left(U^{0}, \Phi^{0}\right)$ in $H^{k}\left(\mathbb{R}^{N}\right) \times H_{\sin }^{k}\left(\mathbb{R}^{N}\right)$, as $\varepsilon \rightarrow 0$, then it is possible to find $T>0$ such that all the corresponding solutions $\left(U_{\varepsilon}, \Phi_{\varepsilon}\right)$ are well-defined on $[0, T]$. This property is necessary in order to make possible a consistent analysis of the limit $\varepsilon \rightarrow 0$.

Statement (i) only holds when the initial data $\left(U_{\varepsilon}^{0}, \Phi_{\varepsilon}^{0}\right)$ satisfy the condition in (16). However, this condition is not a restriction in the limit $\varepsilon \rightarrow 0$. It is satisfied by any fixed pair $\left(U^{0}, \Phi^{0}\right) \in$ $\mathcal{N} \mathcal{V}_{\sin }^{k+1}\left(\mathbb{R}^{N}\right)$ provided that $\varepsilon$ is small enough, so that it is also satisfied by a family of initial data $\left(U_{\varepsilon}^{0}, \Phi_{\varepsilon}^{0}\right)$, which converges towards a pair $\left(U^{0}, \Phi^{0}\right)$ in $H^{k}\left(\mathbb{R}^{N}\right) \times H_{\sin }^{k}\left(\mathbb{R}^{N}\right)$ as $\varepsilon \rightarrow 0$.

Statements (ii) and (iii) in Theorem 3.1 provide two estimates between the previous solutions $\left(U_{\varepsilon}, \Phi_{\varepsilon}\right)$ to $\left(\overrightarrow{\mathrm{H}_{\varepsilon}}\right)$, and an arbitrary global solution $(U, \Phi)$ to $(\mathrm{SGS})$ at the Hamiltonian level. The first one yields an $L^{2}$-control on the difference $\Phi_{\varepsilon}-\Phi$, while the second one, an energetic control on the difference $\left(U_{\varepsilon}, \Phi_{\varepsilon}\right)-(U, \Phi)$. Due to the fact that the difference $\Phi_{\varepsilon}-\Phi$ is not necessarily in $L^{2}\left(\mathbb{R}^{N}\right)$, statement (ii) is restricted to initial conditions satisfying this property.

Finally, statement (iv) bounds the difference between the solutions $\left(U_{\varepsilon}, \Phi_{\varepsilon}\right)$ and $(U, \Phi)$ at the same initial Sobolev level. In this case, we also have to control the maximal time of regularity of the solutions $(U, \Phi)$. This follows from the control from below for $T_{\varepsilon}^{*}$, which is of the same order as the one in $T_{\varepsilon}$.

We then obtain the Sobolev estimate in (iv) of the difference $\left(U_{\varepsilon}, \Phi_{\varepsilon}\right)-(U, \Phi)$ with a loss of three derivatives. Here, the choice of the Sobolev exponents $k>N / 2+3$ is tailored to gain a uniform control on the functions $U_{\varepsilon}-U, \nabla \Phi_{\varepsilon}-\nabla \Phi$ and $\sin \left(\Phi_{\varepsilon}-\Phi\right)$, by the Sobolev embedding theorem.

A loss of derivatives is natural in the context of long-wave regimes; it is related to the terms with first and second-order derivatives in the right-hand side of $\left(\mathrm{H}_{\varepsilon}\right)$. This loss is the reason why the energetic estimate in statement (iii) requires an extra derivative in dimension one, that is the condition $k>N / 2+2$. Using the Sobolev bounds in (19), we can (partly) recover this loss 
by a standard interpolation argument, and deduce an estimate in $H^{\ell}\left(\mathbb{R}^{N}\right) \times H_{\sin }^{\ell+1}\left(\mathbb{R}^{N}\right)$ for any number $\ell<k$. In this case, the error terms are no more of order $\varepsilon^{2}$.

As a by-product of the analysis, we can also analyze the wave regime for the LL equation. This regime is obtained by allowing the parameter $\sigma$ to converge to 0 . Indeed, at least formally, a solution $\left(U_{\varepsilon, \sigma}, \Phi_{\varepsilon, \sigma}\right)$ to $\left(\mathrm{H}_{\varepsilon}\right)$ satisfies the free wave system

$$
\partial_{t} U=\Delta \Phi, \quad \partial_{t} \Phi=U
$$

as $\varepsilon \rightarrow 0$ and $\sigma \rightarrow 0$. In particular, the function $\Phi$ is solution to the wave equation $\partial_{t t} \Phi-\Delta \Phi=0$. The following result provides a rigorous justification for this asymptotic approximation.

Theorem 3.2 ([35]). Let $N \geq 1$ and $k \in \mathbb{N}$, with $k>N / 2+1$, and $0<\varepsilon, \sigma<1$. Consider an initial condition $\left(U_{\varepsilon, \sigma}^{0}, \Phi_{\varepsilon, \sigma}^{0}\right) \in \mathcal{N} \mathcal{V}_{\sin }^{k+2}\left(\mathbb{R}^{N}\right)$ and set

$$
\mathcal{K}_{\varepsilon, \sigma}=\left\|U_{\varepsilon, \sigma}^{0}\right\|_{H^{k}}+\varepsilon\left\|\nabla U_{\varepsilon, \sigma}^{0}\right\|_{H^{k}}+\left\|\nabla \Phi_{\varepsilon, \sigma}^{0}\right\|_{H^{k}}+\sigma^{\frac{1}{2}}\left\|\sin \left(\Phi_{\varepsilon, \sigma}^{0}\right)\right\|_{L^{2}} .
$$

Let $m \in \mathbb{N}$, with $0 \leq m \leq k-2$. Consider similarly an initial condition $\left(U^{0}, \Phi^{0}\right) \in H^{m}\left(\mathbb{R}^{N}\right) \times$ $H^{m-1}\left(\mathbb{R}^{N}\right)$, and denote by $(U, \Phi) \in \mathcal{C}^{0}\left(\mathbb{R}, H^{m-1}\left(\mathbb{R}^{N}\right) \times H^{m}\left(\mathbb{R}^{N}\right)\right)$ the unique corresponding solution to $(\mathrm{FW})$. Then, there exists $C>0$, depending only on $k$ and $N$, such that, if the initial data satisfies the condition $C \varepsilon \mathcal{K}_{\varepsilon, \sigma}^{0} \leq 1$, the following statements hold. Then there exists a positive number

$$
T_{\varepsilon, \sigma} \geq \frac{1}{C \max (\varepsilon, \sigma)\left(1+\mathcal{K}_{\varepsilon, \sigma}\right)^{\max (2, k / 2)}},
$$

such that there is a unique solution $\left(U_{\varepsilon, \sigma}, \Phi_{\varepsilon, \sigma}\right) \in \mathcal{C}^{0}\left(\left[0, T_{\varepsilon, \sigma}\right], \mathcal{N} \mathcal{V}_{\sin }^{k+1}\left(\mathbb{R}^{N}\right)\right)$ to $\left(\mathrm{H}_{\varepsilon}\right)$ with initial data $\left(U_{\varepsilon, \sigma}^{0}, \Phi_{\varepsilon, \sigma}^{0}\right)$. Moreover, if $\Phi_{\varepsilon, \sigma}^{0}-\Phi^{0} \in H^{m}\left(\mathbb{R}^{N}\right)$, then we have the estimate, for any $0 \leq t \leq T_{\varepsilon, \sigma}$,

$$
\begin{aligned}
\left\|U_{\varepsilon, \sigma}(t)-U(t)\right\|_{H^{m-1}} & +\left\|\Phi_{\varepsilon, \sigma}(t)-\Phi(t)\right\|_{H^{m}} \leq C\left(1+t^{2}\right)\left(\left\|U_{\varepsilon, \sigma}^{0}-U^{0}\right\|_{H^{m-1}}\right. \\
& \left.+\left\|\Phi_{\varepsilon, \sigma}^{0}-\Phi^{0}\right\|_{H^{m}}+\max \left(\varepsilon^{2}, \sigma^{1 / 2}\right) \mathcal{K}_{\varepsilon, \sigma}^{0}\left(1+\mathcal{K}_{\varepsilon, \sigma}^{0}\right)^{\max (2, m)}\right) .
\end{aligned}
$$

The wave regime of the LL equation was first derived rigorously by Shatah and Zeng [92, as a special case of the wave regimes for the Schrödinger map equations with values into arbitrary Kähler manifolds. The derivation in 92 relies on energy estimates, which are similar in spirit to the ones we establish in the sequel, and a compactness argument. Getting rid of this compactness argument provides the quantified version of the convergence in Theorem 3.2. This improvement is based on the arguments developed by Béthuel, Danchin and Smets [10] in order to quantify the convergence of the Gross-Pitaevskii equation towards the free wave equation in a similar long-wave regime. Similar arguments were also applied in [26] in order to derive rigorously the (modified) KdV and (modified) KP regimes of the LL equation (see also [49]).

Concerning the proof of Theorem 3.1, the first step is to provide a control on $T_{\max }$. In view of the conditions in statement (ii) of Corollary 2.5, this control can be derived from uniform bounds on the functions $U_{\varepsilon}, \nabla U_{\varepsilon}$ and $\nabla \Phi_{\varepsilon}$. Taking into account the Sobolev embedding theorem and the fact that $k>N / 2+1$, we are left with the computations of energy estimates for the functions $U_{\varepsilon}$ and $\Phi_{\varepsilon}$ in the spaces $H^{k}\left(\mathbb{R}^{N}\right)$ and $H_{\sin }^{k}\left(\mathbb{R}^{N}\right)$, respectively.

In this direction, we recall that the LL energy corresponding to the scaled hydrodynamical system $\left(\mathrm{H}_{\varepsilon}\right)$ writes as

$$
E_{\varepsilon}(t)=\frac{1}{2} \int_{\mathbb{R}^{N}}\left(\varepsilon^{2} \frac{\left|\nabla U_{\varepsilon}\right|^{2}}{1-\varepsilon^{2} U_{\varepsilon}^{2}}+U_{\varepsilon}^{2}+\left(1-\varepsilon^{2} U_{\varepsilon}^{2}\right)\left|\nabla \Phi_{\varepsilon}\right|^{2}+\sigma\left(1-\varepsilon^{2} U_{\varepsilon}^{2}\right) \sin ^{2}\left(\Phi_{\varepsilon}\right)\right) .
$$


Inspired in this formula, we proposed to define an energy of order $k \geq 1$ as

$E_{\varepsilon}^{k}(t)=\frac{1}{2} \sum_{|\alpha|=k-1} \int_{\mathbb{R}^{N}}\left(\varepsilon^{2} \frac{\left|\nabla \partial_{x}^{\alpha} U_{\varepsilon}\right|^{2}}{1-\varepsilon^{2} U_{\varepsilon}^{2}}+\left|\partial_{x}^{\alpha} U_{\varepsilon}\right|^{2}+\left(1-\varepsilon^{2} U_{\varepsilon}^{2}\right)\left|\nabla \partial_{x}^{\alpha} \Phi_{\varepsilon}\right|^{2}+\sigma\left(1-\varepsilon^{2} U_{\varepsilon}^{2}\right)\left|\partial_{x}^{\alpha} \sin \left(\Phi_{\varepsilon}\right)\right|^{2}\right)$.

The factors $1-\varepsilon^{2} U_{\varepsilon}^{2}$ in this expression, as well as the non-quadratic term corresponding to the function $\sin \left(\Phi_{\varepsilon}\right)$, are of substantial importance since they provide a better symmetrization of the energy estimates, by inducing cancellations in the higher order terms. More precisely, we have the following key proposition.

Proposition 3.3. Let $\varepsilon>0$ and $k \in \mathbb{N}$, with $k>N / 2+1$. Consider a solution $\left(U_{\varepsilon}, \Phi_{\varepsilon}\right)$ to $\left(\mathrm{H}_{\varepsilon}\right)$, with $\left(U_{\varepsilon}, \Phi_{\varepsilon}\right) \in \mathcal{C}^{0}\left([0, T], \mathcal{N} \mathcal{V}_{\sin }^{k+3}\left(\mathbb{R}^{N}\right)\right)$ for some $T>0$. Assume that

$$
\inf _{\mathbb{R}^{N} \times[0, T]}\left(1-\varepsilon^{2} U_{\varepsilon}^{2}\right) \geq 1 / 2 .
$$

Then there exists $C>0$, depending only on $k$ and $N$, such that

$$
\begin{aligned}
& {\left[E_{\varepsilon}^{\ell}\right]^{\prime}(t) \leq C \max \left(1, \sigma^{3 / 2}\right)\left(1+\varepsilon^{4}\right)\left(\left\|\sin \left(\Phi_{\varepsilon}(t)\right)\right\|_{L^{\infty}}^{2}+\left\|U_{\varepsilon}(t)\right\|_{L^{\infty}}^{2}+\left\|\nabla \Phi_{\varepsilon}(t)\right\|_{L^{\infty}}^{2}+\left\|\nabla U_{\varepsilon}(t)\right\|_{L^{\infty}}^{2}\right.} \\
& \left.\quad+\left\|d^{2} \Phi_{\varepsilon}(t)\right\|_{L^{\infty}}^{2}+\varepsilon^{2}\left\|d^{2} U_{\varepsilon}(t)\right\|_{L^{\infty}}^{2}+\varepsilon\left\|\nabla \Phi_{\varepsilon}(t)\right\|_{L^{\infty}}\left(\left\|\nabla \Phi_{\varepsilon}(t)\right\|_{L^{\infty}}^{2}+\left\|\nabla U_{\varepsilon}(t)\right\|_{L^{\infty}}^{2}\right)\right) \Sigma_{\varepsilon}^{k+1}(t),
\end{aligned}
$$

for any $t \in[0, T]$ and any $2 \leq \ell \leq k+1$. Here, we have set $\Sigma_{\varepsilon}^{k+1}=\sum_{j=1}^{k+1} E_{\varepsilon}^{j}$.

Thanks to the condition $k>N / 2+1$ and the Sobolev embedding, we get from (17) a differential inequality for $y(t)=\Sigma_{\varepsilon}^{k}$, of the type

$$
y^{\prime}(t) \leq A y^{2}(t)
$$

at least on the interval where $y$ is well-defined and $y(t) \leq 2 y(0)$. Here $A$ is a constant depending on $y(0)$. Integrating $(18)$, we conclude that

$$
y(t) \leq \frac{y(0)}{1-A y(0) t} \leq 2 y(0),
$$

provided that $t \leq 1 /(2 A y(0))$. Using this argument, we deduce from Proposition 3.3, that maximal time $T_{\max }$ is at least of order $1 /\left(\left\|U_{\varepsilon}^{0}\right\|_{H^{k}}+\varepsilon\left\|\nabla U_{\varepsilon}^{0}\right\|_{H^{k}}+\left\|\nabla \Phi_{\varepsilon}^{0}\right\|_{H^{k}}+\left\|\sin \left(\bar{\Phi}_{\varepsilon}^{0}\right)\right\|_{H^{k}}\right)^{2}$, when the initial conditions $\left(U_{\varepsilon}^{0}, \Phi_{\varepsilon}^{0}\right)$ satisfy the inequality in (16). In particular, the dependence of $T_{\max }$ on the small parameter $\varepsilon$ only results from the possible dependence of the pair $\left(U_{\varepsilon}^{0}, \Phi_{\varepsilon}^{0}\right)$ on $\varepsilon$. Choosing suitably these initial conditions, we can assume without loss of generality, that $T_{\max }$ is uniformly bounded from below when $\varepsilon$ tends to 0 , so that analyzing this limit makes sense and we can work in an interval of the form $\left[0, T_{\varepsilon}\right]$. Moreover, we also get the energy estimate on $\left[0, T_{\varepsilon}\right]$ in terms og $\mathcal{K}_{\varepsilon}$ defined in 15,

$$
\left\|U_{\varepsilon}(t)\right\|_{H^{k}}+\varepsilon\left\|\nabla U_{\varepsilon}(t)\right\|_{H^{k}}+\left\|\nabla \Phi_{\varepsilon}(t)\right\|_{H^{k}}+\left\|\sin \left(\Phi_{\varepsilon}(t)\right)\right\|_{H^{k}} \leq C \mathcal{K}_{\varepsilon} .
$$

The final ingredient in the proof of Theorem 3.1 is the consistency of $\left(\mathrm{H}_{\varepsilon}\right)$ with the Sine-Gordon system in the limit $\varepsilon \rightarrow 0$. Indeed, we can rewrite $\left(\mathrm{H}_{\varepsilon}\right]$ as

$$
\partial_{t} U_{\varepsilon}=\Delta \Phi_{\varepsilon}-\frac{\sigma}{2} \sin \left(2 \Phi_{\varepsilon}\right)+\varepsilon^{2} R_{\varepsilon}^{U}, \quad \partial_{t} \Phi_{\varepsilon}=U_{\varepsilon}+\varepsilon^{2} R_{\varepsilon}^{\Phi},
$$

where

$$
\begin{aligned}
& R_{\varepsilon}^{U}=-\operatorname{div}\left(U_{\varepsilon}^{2} \nabla \Phi_{\varepsilon}\right)+\sigma U_{\varepsilon}^{2} \sin \left(\Phi_{\varepsilon}\right) \cos \left(\Phi_{\varepsilon}\right), \\
& R_{\varepsilon}^{\Phi}=-\sigma U_{\varepsilon} \sin ^{2}\left(\Phi_{\varepsilon}\right)-\operatorname{div}\left(\frac{\nabla U_{\varepsilon}}{1-\varepsilon^{2} U_{\varepsilon}^{2}}\right)+\varepsilon^{2} U_{\varepsilon} \frac{\left|\nabla U_{\varepsilon}\right|^{2}}{\left(1-\varepsilon^{2} U_{\varepsilon}^{2}\right)^{2}}-U_{\varepsilon}\left|\nabla \Phi_{\varepsilon}\right|^{2} .
\end{aligned}
$$


In view of the Sobolev control in $(19)$, the remainder terms $R_{\varepsilon}^{U}$ and $R_{\varepsilon}^{\Phi}$ are bounded uniformly with respect to $\varepsilon$ in Sobolev spaces, with a loss of three derivatives. Due to this observation, the differences $u_{\varepsilon}=U_{\varepsilon}-U$ and $\varphi_{\varepsilon}=\Phi_{\varepsilon}-\Phi$ between a solution $\left(U_{\varepsilon}, \Phi_{\varepsilon}\right)$ to $\left(\mathrm{H}_{\varepsilon}\right)$ and a solution $(U, \Phi)$ to (SGS) are expected to be of order $\varepsilon^{2}$, if the corresponding initial conditions are close enough. The proof of this claim would be immediate if the system 20 would not contain the nonlinear term $\sin \left(2 \Phi_{\varepsilon}\right)$. Due to this extra term, we have to apply a Gronwall argument in order to control the differences $u_{\varepsilon}$ and $\varphi_{\varepsilon}$. This can be done since $v_{\varepsilon}$ and $\varphi_{\varepsilon}$ satisfy

$$
\partial_{t} v_{\varepsilon}=\Delta \varphi_{\varepsilon}-\sigma \sin \left(\varphi_{\varepsilon}\right) \cos \left(\Phi_{\varepsilon}+\Phi\right)+\varepsilon^{2} R_{\varepsilon}^{U}, \quad \partial_{t} \varphi_{\varepsilon}=v_{\varepsilon}+\varepsilon^{2} R_{\varepsilon}^{\Phi},
$$

so that we can perform energy estimates as before.

\subsection{The cubic NLS regime}

We now focus on the cubic Schrödinger equation, which is obtained in a regime of strong easy-axis anisotropy of equation (14). For this purpose, we consider a uniaxial material in the direction corresponding to the vector $\boldsymbol{e}_{2}$ and we fix the anisotropy parameters as $\lambda_{1}=\lambda_{3}=1 / \varepsilon$. For this choice, let us introduce the complex-valued function $\Psi_{\varepsilon}$ given by

$$
\Psi_{\varepsilon}(x, t)=\varepsilon^{-1 / 2} \check{m}(x, t) e^{i t / \varepsilon}, \quad \text { with } \check{m}=m_{1}+i m_{3},
$$

associated with a solution $\boldsymbol{m}$ of $(14)$. This function is of order 1 in the regime where the map $\check{m}$ is of order $\varepsilon^{\frac{1}{2}}$. When $\varepsilon$ is small enough, the function $m_{2}$ does not vanish in this regime, since the solution $\boldsymbol{m}$ is valued into the sphere $\mathbb{S}^{2}$. Assuming that $m_{2}$ is everywhere positive, it is given by the formula

$$
m_{2}=\left(1-\varepsilon\left|\Psi_{\varepsilon}\right|^{2} \mid\right)^{\frac{1}{2}},
$$

and the function $\Psi_{\varepsilon}$ is a solution to the nonlinear Schrödinger equation

$$
i \partial_{t} \Psi_{\varepsilon}+\left(1-\varepsilon\left|\Psi_{\varepsilon}\right|^{2}\right)^{1 / 2} \Delta \Psi_{\varepsilon}+\frac{\left|\Psi_{\varepsilon}\right|^{2}}{1+\left(1-\varepsilon\left|\Psi_{\varepsilon}\right|^{2}\right)^{1 / 2}} \Psi_{\varepsilon}+\varepsilon \operatorname{div}\left(\frac{\left\langle\Psi_{\varepsilon}, \nabla \Psi_{\varepsilon}\right\rangle_{\mathbb{C}}}{\left(1-\varepsilon\left|\Psi_{\varepsilon}\right|^{2}\right)^{1 / 2}}\right) \Psi_{\varepsilon}=0
$$

where $\left\langle z_{1}, z_{2}\right\rangle_{\mathbb{C}}=\operatorname{Re}\left(z_{1} \bar{z}_{2}\right)$. As $\varepsilon \rightarrow 0$, the formal limit is therefore the focusing cubic Schrödinger equation

$$
i \partial_{t} \Psi+\Delta \Psi+\frac{1}{2}|\Psi|^{2} \Psi=0 .
$$

The goal is to justify rigorously this cubic Schrödinger regime of the LL equation. We recall that (CS) is locally well-posed in $H^{k}\left(\mathbb{R}^{N}\right)$, for $k \in \mathbb{N}$; we refer to [24] for an extended review on this subject. Going on with our rigorous derivation of the cubic Schrödinger regime, we now express the local well-posedness result in Theorem 2.2 in terms of the nonlinear Schrödinger equation $\left(\mathrm{NLS}_{\varepsilon}\right)$ satisfied by the rescaled function $\Psi_{\varepsilon}$.

Corollary 3.4 ([36]). Let $\varepsilon>0$, and $k \in \mathbb{N}$, with $k>N / 2+1$. Consider a function $\Psi_{\varepsilon}^{0} \in H^{k}\left(\mathbb{R}^{N}\right)$ such that

$$
\varepsilon^{1 / 2}\left\|\Psi_{\varepsilon}^{0}\right\|_{L^{\infty}}<1
$$

Then there exist $T_{\varepsilon}>0$ and a unique solution $\Psi_{\varepsilon} \in L^{\infty}\left([0, T], H^{k}\left(\mathbb{R}^{N}\right)\right)$ to $\left(\mathrm{NLS}_{\varepsilon}\right)$, for any $t \in\left(0, T_{\varepsilon}\right)$. Moreover, the flow map $\Psi_{\varepsilon}^{0} \mapsto \Psi_{\varepsilon}$ is Lipschitz continuous from $H^{k}\left(\mathbb{R}^{N}\right)$ to $\mathcal{C}^{0}\left([0, T], H^{k-1}\left(\mathbb{R}^{N}\right)\right)$ for any $T \in\left(0, T_{\varepsilon}\right)$ and the nonlinear Schrödinger energy $\mathfrak{E}_{\varepsilon}$ given by

$$
\mathfrak{E}_{\varepsilon}\left(\Psi_{\varepsilon}\right)=\frac{1}{2} \int_{\mathbb{R}^{N}}\left(\left|\Psi_{\varepsilon}\right|^{2}+\varepsilon\left|\nabla \Psi_{\varepsilon}\right|^{2}+\frac{\varepsilon^{2}\left\langle\Psi_{\varepsilon}, \nabla \Psi_{\varepsilon}\right\rangle_{\mathbb{C}}^{2}}{1-\varepsilon\left|\Psi_{\varepsilon}\right|^{2}}\right)
$$

is conserved along the flow. 
We are now in position to state the main result concerning the rigorous derivation of the cubic Schrödinger regime of the LL equation.

Theorem $3.5([36])$. Let $0<\varepsilon<1$, and $k \in \mathbb{N}$, with $k>N / 2+2$. Consider two initial conditions $\Psi^{0} \in H^{k}\left(\mathbb{R}^{N}\right)$ and $\Psi_{\varepsilon}^{0} \in H^{k+3}\left(\mathbb{R}^{N}\right)$, and set

$$
\mathcal{S}_{\varepsilon}=\left\|\Psi^{0}\right\|_{H^{k}}+\left\|\Psi_{\varepsilon}^{0}\right\|_{H^{k}}+\varepsilon^{\frac{1}{2}}\left\|\nabla \Psi_{\varepsilon}^{0}\right\|_{\dot{H}^{k}}+\varepsilon\left\|\Delta \Psi_{\varepsilon}^{0}\right\|_{\dot{H}^{k}} .
$$

There is $A>0$, depending only on $k$, such that, if the initial data $\Psi^{0}$ and $\Psi_{\varepsilon}^{0}$ satisfy the condition

$$
A \varepsilon^{\frac{1}{2}} \mathcal{S}_{\varepsilon} \leq 1
$$

then there exists a time $T_{\varepsilon} \geq \frac{1}{A \mathcal{K}_{\varepsilon}^{2}}$, such that both the unique solution $\Psi_{\varepsilon}$ to $\mathrm{NLS}_{\varepsilon}$ with initial data $\Psi_{\varepsilon}^{0}$, and the unique solution $\Psi$ to (CS) with initial data $\Psi^{0}$ are well-defined on the time interval $\left[0, T_{\varepsilon}\right]$. Moreover, we have the error estimate, for any $t \in\left[0, T_{\varepsilon}\right]$,

$$
\left\|\Psi_{\varepsilon}(t)-\Psi(t)\right\|_{H^{k-2}} \leq\left(\left\|\Psi_{\varepsilon}^{0}-\Psi^{0}\right\|_{H^{k-2}}+A \varepsilon \mathcal{S}_{\varepsilon}\left(1+\mathcal{S}_{\varepsilon}^{3}\right)\right) e^{A \mathcal{S}_{\varepsilon}^{2} t} .
$$

In this manner, Theorem 3.5 establishes rigorously the convergence of the LL equation towards the cubic Schrödinger equation in any dimension. It is certainly possible to show only convergence under weaker assumptions by using compactness arguments as for the derivation of similar asymptotic regimes (see e.g. [92, 27, 49] concerning Schrödinger-like equations).

Observe that smooth solutions for both the LL and the cubic Schrödinger equations are known to exist when the integer $k$ satisfies the condition $k>N / 2+1$. The additional assumption $k>N / 2+2$ in Theorem 3.5 is related to the fact that the proof of (22) requires a uniform control of the difference $\Psi_{\varepsilon}-\Psi$, which follows from the Sobolev embedding theorem of $H^{k-2}\left(\mathbb{R}^{N}\right)$ into $L^{\infty}\left(\mathbb{R}^{N}\right)$

Finally, the loss of two derivatives in the error estimate $(22)$ can be partially recovered by combining standard interpolation theory. Under the assumptions of Theorem 3.5, the solutions $\Psi_{\varepsilon}$ converge towards the solution $\Psi$ in $\mathcal{C}^{0}\left(\left[0, T_{\varepsilon}\right], H^{s}\left(\mathbb{R}^{N}\right)\right)$ for any $0 \leq s<k$, when $\Psi_{\varepsilon}^{0}$ tends to $\Psi^{0}$ in $H^{k+2}\left(\mathbb{R}^{N}\right)$ as $\varepsilon \rightarrow 0$, but the error term is not necessarily of order $\varepsilon$ due to the interpolation process.

Note here that condition (21) is not really restrictive in order to analyze such a convergence. At least when $\Psi_{\varepsilon}^{0}$ tends to $\Psi^{0}$ in $H^{k+2}\left(\mathbb{R}^{N}\right)$ as $\varepsilon \rightarrow 0$, the quantity $\mathcal{S}_{\varepsilon}$ tends to twice the norm $\left\|\Psi^{0}\right\|_{H^{k}}$ in the limit $\varepsilon \rightarrow 0$, so that condition (21) is always fulfilled. Moreover, the error estimate 22 is available on a time interval of order $1 /\left\|\Psi^{0}\right\|_{H^{k}}^{2}$, which is similar to the minimal time of existence of the smooth solutions to the cubic Schrödinger equation.

The proof of Theorem 3.5 is similar to the proof of Theorem 3.1. It relies on the consistency between the Schrödinger equations $\left(\mathrm{NLS}_{\varepsilon}\right)$ and (CS) in the limit $\varepsilon \rightarrow 0$. Indeed, we can recast $\left(\mathrm{NLS}_{\varepsilon}\right)$ as

$$
i \partial_{t} \Psi_{\varepsilon}+\Delta \Psi_{\varepsilon}+\frac{1}{2}\left|\Psi_{\varepsilon}\right|^{2} \Psi_{\varepsilon}=\varepsilon \mathcal{R}_{\varepsilon}
$$

where the remainder term $\mathcal{R}_{\varepsilon}$ is given by

$$
\mathcal{R}_{\varepsilon}=\frac{\left|\Psi_{\varepsilon}\right|^{2}}{1+\left(1-\varepsilon\left|\Psi_{\varepsilon}\right|^{2}\right)^{\frac{1}{2}}} \Delta \Psi_{\varepsilon}-\frac{\left|\Psi_{\varepsilon}\right|^{4}}{2\left(1+\left(1-\varepsilon\left|\Psi_{\varepsilon}\right|^{2}\right)^{\frac{1}{2}}\right)^{2}} \Psi_{\varepsilon}-\operatorname{div}\left(\frac{\left\langle\Psi_{\varepsilon}, \nabla \Psi_{\varepsilon}\right\rangle_{\mathbb{C}}}{\left(1-\varepsilon\left|\Psi_{\varepsilon}\right|^{2}\right)^{\frac{1}{2}}}\right) \Psi_{\varepsilon}
$$

In order to establish the convergence towards the cubic Schrödinger equation, the main goal is to control the remainder term $\mathcal{R}_{\varepsilon}$ on a time interval $\left[0, T_{\varepsilon}\right]$ as long as possible. In particular, we have to show that the maximal time $T_{\varepsilon}$ for this control does not vanish in the limit $\varepsilon \rightarrow 0$. The 
main argument is to perform suitable energy estimates on the solutions $\Psi_{\varepsilon}$ to $\left(\mathrm{NLS}_{\varepsilon}\right)$. These estimates provide Sobolev bounds for the remainder term $\mathcal{R}_{\varepsilon}$, which are used to control the differences $u_{\varepsilon}=\Psi_{\varepsilon}-\Psi$ with respect to the solutions $\Psi$ to (CS). This further control is also derived from energy estimates.

Concerning the estimates of the solutions $\Psi_{\varepsilon}$, we rely on the equivalence with the solutions $\boldsymbol{m}$ to (14). However, the estimates given in Section 2 are not enough in this case. It is crucial to refine the estimate (12), which can be done when $\lambda_{1}=\lambda_{3}$.

Proposition 3.6. Let $0<\varepsilon<1$, and $k \in \mathbb{N}$, with $k>N / 2+1$. Assume that $\lambda_{1}=\lambda_{3}=1 / \varepsilon$, and that $\boldsymbol{m}$ is a solution to (14) in $\mathcal{C}^{0}\left([0, T], \mathcal{E}^{k+4}\left(\mathbb{R}^{N}\right)\right)$, with $\partial_{t} m \in \mathcal{C}^{0}\left([0, T], H^{k+2}\left(\mathbb{R}^{N}\right)\right)$. Given any integer $2 \leq \ell \leq k+2$, the energies $E_{\ell}$ are of class $\mathcal{C}^{1}$ on $[0, T]$, and there exists $C_{k}>0$, depending possibly on $k$, but not on $\varepsilon$, such that their derivatives satisfy

$$
E_{\ell}^{\prime}(t) \leq \frac{C_{k}}{\varepsilon}\left(\left\|m_{1}(t)\right\|_{L^{\infty}}^{2}+\left\|m_{3}(t)\right\|_{L^{\infty}}^{2}+\|\nabla \boldsymbol{m}(t)\|_{L^{\infty}}^{2}\right)\left(E_{\ell}(t)+E_{\ell-1}(t)\right),
$$

for any $t \in[0, T]$. Here we have set $E_{1}(t)=E(\boldsymbol{m}(t))$, the LL energy.

As for the proof of Proposition 2.3, the estimates in Proposition 3.6 rely on the identity (11), that in the case $\lambda_{1}=\lambda_{3}=1 / \varepsilon$ can be simplified. In contrast with the estimate $(12)$, the multiplicative factor in the right-hand side of (23) now only depends on the uniform norms of the functions $m_{1}, m_{3}$ and $\nabla \boldsymbol{m}$. This property is key in order to use these estimates in the cubic Schrödinger regime.

Finally, it is necessary to find a high order energy, with suitable cancellation properties to obtain good energy estimates. The energy proposed in [35], which allows us to conclude as in the sine-Gordon equation, is

$$
\begin{aligned}
\mathfrak{E}_{\varepsilon}^{k}(t)= & \left\|\Psi_{\varepsilon}\right\|_{\dot{H}^{k-2}}^{2}+\left\|\varepsilon \partial_{t} \Psi_{\varepsilon}-i \Psi_{\varepsilon}\right\|_{\dot{H}^{k-2}}^{2}+\varepsilon^{2}\left\|\Delta \Psi_{\varepsilon}\right\|_{\dot{H}^{k-2}}^{2} \\
& +\varepsilon\left(\left\|\partial_{t}\left(1-\varepsilon\left|\Psi_{\varepsilon}\right|^{2}\right)^{\frac{1}{2}}\right\|_{\dot{H}^{k-2}}^{2}+\left\|\Delta\left(1-\varepsilon\left|\Psi_{\varepsilon}\right|^{2}\right)^{\frac{1}{2}}\right\|_{\dot{H}^{k-2}}^{2}+2\left\|\nabla \Psi_{\varepsilon}\right\|_{\dot{H}^{k-2}}^{2}\right),
\end{aligned}
$$

for any $k \geq 2$. We refer to [35] for detailed computations.

\section{Stability of sum of solitons}

In dimension one, the LL equation is completely integrable by means of the inverse scattering method [43] and, using this technique, explicit solitons and multisolitons solutions can be constructed [17]. We consider in this section equation (13), i.e. the one-dimensional easy-plane LL equation. By a change of variable, we assume that $\lambda_{3}=1$.

We say that a soliton for (13) is a traveling wave of the form $\boldsymbol{m}(x, t)=\boldsymbol{u}(x-c t)$. The nonconstant solitons are explicitly given by

$$
\boldsymbol{u}_{c}(x)=\left(c \operatorname{sech}\left(\sqrt{1-c^{2}} x\right), \tanh \left(\sqrt{1-c^{2}} x\right), \sqrt{1-c^{2}} \operatorname{sech}\left(\sqrt{1-c^{2}} x\right)\right), \quad|c|<1,
$$

up to the invariances of the equation, i.e. translations, rotations around the axis $x_{3}$ and orthogonal symmetries with respect to any line in the plane $x_{3}=0$. Thus a soliton with speed $c$ may be also written as

$$
\boldsymbol{u}_{c, a, \theta, s}(x)=\left(\cos (\theta)\left[\boldsymbol{u}_{c}\right]_{1}-s \sin (\theta)\left[\boldsymbol{u}_{c}\right]_{2}, \sin (\theta)\left[\boldsymbol{u}_{c}\right]_{1}+s \cos (\theta)\left[\boldsymbol{u}_{c}\right]_{2}, s\left[\boldsymbol{u}_{c}\right]_{3}\right)(x-a),
$$

with $a \in \mathbb{R}, \theta \in \mathbb{R}$ and $s \in\{ \pm 1\}$. We refer to [34, 35, 36] for more properties of solitons for the LL equation (3). 
In addition, using the integrability of the equation and by means of the inverse scattering method, for any $M \in \mathbb{N}^{*}$, it can be also computed explicit solutions to (13) that behave like a sum of $M$ decoupled solitons as $t \rightarrow \infty$. These solutions are often called $M$-solitons or simply multisolitons (see e.g. [17, Section 10] for their explicit formula).

We can define properly the solitons in the hydrodynamical framework when $c \neq 0$, since the function $\check{u}_{c}=\left[\boldsymbol{u}_{c}\right]_{1}+i\left[\boldsymbol{u}_{c}\right]_{2}$ does not vanish. More precisely, we recall that for a function $\boldsymbol{u}: \mathbb{R} \rightarrow \mathbb{S}^{2}$ such that $|\boldsymbol{u}| \neq 0$, we set $\breve{u}=\left(1-u_{3}^{2}\right)^{1 / 2} i \exp (-i \varphi)$, and we define the hydrodynamical variables $v=u_{3}$ and $w=-\partial_{x} \varphi$. Thus, equation (13) recasts as in (H1d), and the soliton $\boldsymbol{u}_{c}$ in the hydrodynamical variables $\mathfrak{v}_{c}=\left(v_{c}, w_{c}\right)$ is given by

$$
v_{c}(x)=\sqrt{1-c^{2}} \operatorname{sech}\left(\sqrt{1-c^{2}} x\right), \text { and } w_{c}(x)=\frac{c v_{c}(x)}{1-v_{c}(x)^{2}}=\frac{c \sqrt{1-c^{2}} \cosh \left(\sqrt{1-c^{2}} x\right)}{\sinh \left(\sqrt{1-c^{2}} x\right)^{2}+c^{2}} .
$$

Therefore, the only remaining invariances of solitons in this framework are translations and the opposite map $(v, w) \mapsto(-v,-w)$. Any soliton with speed $c$ may be then written as $\mathfrak{v}_{c, a, s}(x)=s \mathfrak{v}_{c}(x-a)=\left(s v_{c}(x-a), s w_{c}(x-a)\right)$, with $a \in \mathbb{R}$ and $s \in\{ \pm 1\}$.

Our goal in this section is to establish the stability of a single soliton $u_{c}$ along the LL flow. More generally, we will also consider the case of a sum of solitons. In the original framework, defining this sum is not so easy, since the sum of unit vectors in $\mathbb{R}^{3}$ does not necessarily remain in $\mathbb{S}^{2}$. In the hydrodynamical framework, this difficulty does not longer arise. We can define a sum of $M$ solitons $S_{\mathfrak{c}, \mathfrak{a}, \mathfrak{s}}$ as

$$
\boldsymbol{S}_{\mathfrak{c}, \mathfrak{a}, \mathfrak{s}}=\left(V_{\mathfrak{c}, \mathfrak{a}, \mathfrak{s}}, W_{\mathfrak{c}, \mathfrak{a}, \mathfrak{s}}\right)=\sum_{j=1}^{M} \mathfrak{v}_{c_{j}, a_{j}, s_{j}}
$$

with $M \in \mathbb{N}^{*}, \mathfrak{c}=\left(c_{1}, \ldots, c_{M}\right), \mathfrak{a}=\left(a_{1}, \ldots, a_{M}\right) \in \mathbb{R}^{M}$, and $\mathfrak{s}=\left(s_{1}, \ldots, s_{M}\right) \in\{ \pm 1\}^{M}$. However, we have to restrict the analysis to speeds $c_{j} \neq 0$, since the function $\check{u}_{0}$, associated with the black soliton, vanishes at the origin.

Coming back to the original framework, we can define properly a corresponding sum of solitons $\boldsymbol{R}_{\mathfrak{c}, \mathfrak{a}, \mathfrak{s}}$, when the third component of $\boldsymbol{S}_{\mathfrak{c}, \mathfrak{a}, \mathfrak{s}}$ does not reach the values \pm 1 . Due to the exponential decay of the functions $v_{c}$ and $w_{c}$, this assumption is satisfied at least when the positions $a_{j}$ are sufficiently separated, i.e. when the solitons are decoupled. In this case, the sum $\boldsymbol{R}_{\mathfrak{c}, \mathfrak{a}, \mathfrak{s}}$ is given, up to a phase factor, by the expression

$$
\boldsymbol{R}_{\mathfrak{c}, \mathfrak{a}, \mathfrak{s}}=\left(\left(1-V_{\mathfrak{c}, \mathfrak{a}, \mathfrak{s}}^{2}\right)^{\frac{1}{2}} \cos \left(\Phi_{\mathfrak{c}, \mathfrak{a}, \mathfrak{s}}\right),\left(1-V_{\mathfrak{c}, \mathfrak{a}, \mathfrak{s}}^{2}\right)^{\frac{1}{2}} \sin \left(\Phi_{\mathfrak{c}, \mathfrak{a}, \mathfrak{s}}\right), V_{\mathfrak{c}, \mathfrak{a}, \mathfrak{s}}\right), \quad \text { with } \Phi_{\mathfrak{c}, \mathfrak{a}, \mathfrak{s}}(x)=\int_{0}^{x} W_{\mathfrak{c}, \mathfrak{a}, \mathfrak{s}}(y) d y,
$$

for any $x \in \mathbb{R}$. This definition presents the advantage to provide a quantity with values on the sphere $\mathbb{S}^{2}$. On the other hand, it is only defined under restrictive assumptions on the speeds $c_{j}$ and positions $a_{j}$. Moreover, it does not take into account the geometric invariance with respect to rotations around the axis $x_{3}$.

\subsection{Orbital stability in the energy space}

In the sequel, our main results are proved in the hydrodynamical framework. We establish that, if the initial positions $a_{j}^{0}$ are well-separated and the initial speeds $c_{j}^{0}$ are ordered according to the initial positions $a_{j}^{0}$, then the solution corresponding to a chain of solitons at initial time, that is a perturbation of a sum of solitons $S_{\mathfrak{c}^{0}, \mathfrak{a}^{0}, \mathfrak{s}^{0}}$, is uniquely defined, and that it remains a chain of solitons for any positive time. 
Let us recall that Theorem 2.6 provides the existence and uniqueness of a continuous flow for $(\mathrm{H} 1 \mathrm{~d})$ in the nonvanishing energy space $\mathcal{N} \mathcal{V}(\mathbb{R})$. To our knowledge, the question of the global existence (in the hydrodynamical framework) of the local solution $\mathfrak{v}$ is open. In the sequel, we by-pass this difficulty using the stability of a well-prepared sum of solitons $\boldsymbol{S}_{\mathfrak{c}, \mathfrak{a}, \mathfrak{s}}$. Since the solitons in such a sum have exponential decay by (24), and are sufficiently well-separated, the sum $\boldsymbol{S}_{\mathfrak{c}, \mathfrak{a}, \mathfrak{s}}$ belongs to $\mathcal{N} \mathcal{V}(\mathbb{R})$. Invoking the Sobolev embedding theorem, this remains true for a small perturbation in $H^{1}(\mathbb{R}) \times L^{2}(\mathbb{R})$. As a consequence, the global existence for a well-prepared sum of solitons follows from its stability by applying a continuation argument.

Concerning the stability of sums of solitons, our main result is

Theorem 4.1 ([34]). Let $\mathfrak{s}^{*} \in\{ \pm 1\}^{M}$ and $\mathfrak{c}^{*}=\left(c_{1}^{*}, \ldots, c_{M}^{*}\right) \in((-1,1) \backslash\{0\})^{M}$ such that $c_{1}^{*}<c_{2}^{*}<\cdots<c_{M}^{*}$. There exist positive numbers $\alpha^{*}, L^{*}, \nu$ and $A$, depending only on $\mathfrak{c}^{*}$ such that, if $\mathfrak{v}^{0} \in \mathcal{N} \mathcal{V}(\mathbb{R})$ satisfies the condition

$$
\alpha:=\left\|\mathfrak{v}^{0}-S_{\mathfrak{c}^{*}, \mathfrak{a}^{0}, \mathfrak{s}^{*}}\right\|_{H^{1} \times L^{2}} \leq \alpha^{*},
$$

for points $\mathfrak{a}^{0}=\left(a_{1}^{0}, \ldots, a_{M}^{0}\right) \in \mathbb{R}^{M}$ such that $L^{0}:=\min \left\{a_{j+1}^{0}-a_{j}^{0}, 1 \leq j \leq M-1\right\} \geq L^{*}$, then the solution $\mathfrak{v}$ to $(\overline{\mathrm{H} 1 \mathrm{~d}})$ with initial condition $\mathfrak{v}^{0}$ is globally well-defined on $\mathbb{R}_{+}$, and there exists a function $\boldsymbol{a}=\left(a_{1}, \ldots, a_{M}\right) \in \mathcal{C}^{1}\left(\mathbb{R}_{+}, \mathbb{R}^{M}\right)$ such that, for any $t \geq 0$,

$$
\sum_{j=1}^{M}\left|a_{j}^{\prime}(t)-c_{j}^{*}\right| \leq A\left(\alpha+e^{-\nu L^{0}}\right), \quad \text { and }\left\|\mathfrak{v}(\cdot, t)-\boldsymbol{S}_{\mathfrak{c}^{*}, \boldsymbol{a}(t), \mathfrak{s}^{*}}\right\|_{H^{1} \times L^{2}} \leq A\left(\alpha+e^{-\nu L^{0}}\right) .
$$

Theorem 4.1 provides the orbital stability of well-prepared sums of solitons with different, nonzero speeds for positive time. The sums are well-prepared in the sense that their positions at initial time are well-separated and ordered according to their speeds. As a consequence, the solitons are more and more separated along the LL flow (see estimate (25)) and their interactions become weaker and weaker. The stability of the chain then results from the orbital stability of each single soliton in the chain.

As a matter of fact, the orbital stability of a single soliton appears as a special case of Theorem 4.1 when $M=1$. In this case, stability occurs for both positive and negative times due to the time reversibility of the LL equation. Time reversibility also provides the orbital stability of reversely well-prepared chains of solitons for negative time. The analysis of stability for both negative and positive time is more involved. It requires a deep understanding of the possible interactions between the solitons in the chain (see [83, 84, for such an analysis in the context of the $\mathrm{KdV}$ equation). This issue is of particular interest because of the existence of multisolitons.

Special chains of solitons are indeed provided by the exact multisolitons. However, there is a difficulty to define them properly in the hydrodynamical framework. Indeed, multisolitons can reach the values \pm 1 at some times. On the other hand, an arbitrary multisoliton becomes well-prepared for large time in the sense that the individual solitons are ordered according to their speeds and well-separated (see e.g. [17, Section 10]).

If we consider a perturbation of an arbitrary multisoliton at initial time, our theorem does not guarantee that a perturbation of this multisoliton remains a perturbation of a multisoliton for large time. In fact, this property would follow from the continuity with respect to the initial datum of LL equation in the energy space, which remains, to our knowledge, an open question. We remark that Theorem 4.1 only shows the orbital stability of the multisolitons, which do not reach the values \pm 1 for any positive time.

To our knowledge, the orbital stability of the soliton $\boldsymbol{u}_{0}$ remains an open question. In the context of the Gross-Pitaevskii equation, the orbital stability of the vanishing soliton (often 
called black soliton) was proved in [12, 47]. Part of the analysis in this further context certainly extends to the soliton $\boldsymbol{u}_{0}$ of the LL equation.

Let us remark that in case $\lambda_{3}=0$, there is no traveling-wave solution to (13) with nonzero speed and finite energy. However, breather-like solutions were found to exist in [73], and their numerical stability was investigated in 99. In the easy-axis case, there are traveling-wave solutions (see e.g. [18]), but their third coordinate $m_{3}(x)$ converges to \pm 1 as $|x| \rightarrow+\infty$. This prevents from invoking the hydrodynamical formulation, and thus from using the strategy developed below in order to prove their orbital stability. In the rest of this section, we

We present now the main elements in the proof of Theorem 4.1, restricting our attention to the analysis of a single soliton. We underline that these arguments do not make use of the inverse scattering transform. Instead, they rely on the Hamiltonian structure of the LL equation, in particular, on the conservation laws for the energy and momentum. As a consequence, these arguments can presumably be extended to nonintegrable equations similar to the hydrodynamical LL equation.

The strategy of the proof of Theorem 4.1 is reminiscent of the one developed to tackle the stability of well-prepared chains of solitons for the generalized KdV equations [85, the nonlinear Schrödinger equations [86], or the Gross-Pitaevskii equation [14]. A key ingredient in the proof is the minimizing nature of the soliton $\mathfrak{v}_{c}$, which can be constructed as the solution of the minimization problem

$$
E\left(\mathfrak{v}_{c}\right)=\min \left\{E(\mathfrak{v}) \mid \mathfrak{v} \in \mathcal{N} \mathcal{V}(\mathbb{R}) \text { s.t. } P(\mathfrak{v})=P\left(\mathfrak{v}_{c}\right)\right\},
$$

where we recall that the energy and the momentum of $\mathfrak{v}=(v, w)$, are given by

$$
E(\mathfrak{v})=\frac{1}{2} \int_{\mathbb{R}}\left(\frac{\left(v^{\prime}\right)^{2}}{1-v^{2}}+\left(1-v^{2}\right) w^{2}+v^{2}\right), \text { and } P(\mathfrak{v})=\int_{\mathbb{R}} v w .
$$

This characterization results from the compactness of the minimizing sequences for (26), and the classification of solitons in (24). The compactness of minimizing sequences can be proved following the arguments developed for a similar problem in the context of the Gross-Pitaevskii equation [11, 37].

The Euler-Lagrange equation for (26) reduces to the identity $E^{\prime}\left(\mathfrak{v}_{c}\right)=c P^{\prime}\left(\mathfrak{v}_{c}\right)$, where the speed $c$ appears as the Lagrange multiplier of the minimization problem. The minimizing energy is equal to $E\left(\mathfrak{v}_{c}\right)=2\left(1-c^{2}\right)^{\frac{1}{2}}$, while the momentum of the soliton $\mathfrak{v}_{c}$ is given by $P\left(\mathfrak{v}_{c}\right)=2 \arctan \left(\left(1-c^{2}\right)^{\frac{1}{2}} / c\right)$, for $c \neq 0$. An important consequence is the inequality

$$
\frac{d}{d c}\left(P\left(\mathfrak{v}_{c}\right)\right)=-\frac{2}{\left(1-c^{2}\right)^{\frac{1}{2}}}<0,
$$

which is related to the Grillakis-Shatah-Strauss condition (see e.g. [54]) for the orbital stability of a soliton. As a matter of fact, we can use inequality (27) to establish the coercivity of the quadratic form

$$
Q_{c}=E^{\prime \prime}\left(\mathfrak{v}_{c}\right)-c P^{\prime \prime}\left(\mathfrak{v}_{c}\right),
$$

under suitable orthogonality conditions. More precisely, we show

Proposition 4.2. Let $c \in(-1,1) \backslash\{0\}$. There exists $\Lambda_{c}>0$, such that

$$
Q_{c}(\varepsilon) \geq \Lambda_{c}\|\varepsilon\|_{H^{1} \times L^{2}}^{2},
$$

for any pair $\varepsilon \in H^{1}(\mathbb{R}) \times L^{2}(\mathbb{R})$ satisfying the two orthogonality conditions

$$
\left\langle\partial_{x} \mathfrak{v}_{c}, \varepsilon\right\rangle_{L^{2} \times L^{2}}=\left\langle P^{\prime}\left(\mathfrak{v}_{c}\right), \varepsilon\right\rangle_{L^{2} \times L^{2}}=0 .
$$

Moreover, the map $c \mapsto \Lambda_{c}$ is uniformly bounded from below on any compact subset of $(-1,1) \backslash\{0\}$. 
The first orthogonality condition in (29) originates in the invariance with respect to translations of (H1d). Due to this invariance, the pair $\partial_{x} \mathfrak{v}_{c}$ lies in the kernel of $Q_{c}$. The quadratic form $Q_{c}$ also owns a unique negative direction, which is related to the constraint in (26). This direction is controlled by the second orthogonality condition in (29).

As a consequence of Proposition 4.2, the functional $F_{c}(\mathfrak{v})=E(\mathfrak{v})-c P(\mathfrak{v})$, controls any perturbation $\varepsilon=\mathfrak{v}-\mathfrak{v}_{c}$ satisfying the two orthogonality conditions in (29). More precisely, we derive from the Euler-Lagrange equation and (28) that

$$
F_{c}\left(\mathfrak{v}_{c}+\varepsilon\right)-F_{c}\left(\mathfrak{v}_{c}\right) \geq \Lambda_{c}\|\varepsilon\|_{H^{1} \times L^{2}}^{2}+\mathcal{O}\left(\|\varepsilon\|_{H^{1} \times L^{2}}^{3}\right),
$$

as $\|\varepsilon\|_{H^{1} \times L^{2}} \rightarrow 0$. Since the energy $E(\mathfrak{v})$ and the momentum $P(\mathfrak{v})$ are conserved along the flow, the left-hand side of (30) remains small for all time if it was small at the initial time. As a consequence of $(30)$, the perturbation $\varepsilon$ remains small for all time, which implies the stability of $\mathfrak{v}_{c}$. We refer to 34 for more detail about the proof of Theorem 4.1.

\subsection{Asymptotic stability}

We consider now the long-time asymptotics of a solution to (13), with initial condition a perturbation of a soliton. We would like to determine conditions such that the solution converges to a (possible different) soliton. Let us remark that the convergence as $t \rightarrow \infty$ cannot hold in the energy space. For instance, we could consider a solution $\mathfrak{v}$ to $(\mathrm{H} 1 \mathrm{~d})$ with an initial condition $\mathfrak{v}^{0} \in \mathcal{N} \mathcal{V}(\mathbb{R})$, such that $\mathfrak{v}$ converges to a hydrodynamical soliton $\mathfrak{v}_{c}$ in the norm $\|\cdot\|_{H^{1} \times L^{2}}$, as $t \rightarrow \infty$. By the continuity of the energy and the momentum (with respect to this norm), we have

$$
E(\mathfrak{v}(\cdot, t)) \rightarrow E\left(\mathfrak{v}_{c}\right) \quad \text { and } \quad P(\mathfrak{v}(\cdot, t)) \rightarrow P\left(\mathfrak{v}_{c}\right),
$$

as $t \rightarrow \infty$. Since these quantities are conserved by the flow, we conclude that $E\left(\mathfrak{v}^{0}\right)=E\left(\mathfrak{v}_{c}\right)$ and $P\left(\mathfrak{v}^{0}\right)=P\left(\mathfrak{v}_{c}\right)$. Thus, the variational characterization of solitons implies that $\mathfrak{v}^{0}$ must be a soliton. Therefore, the only solutions that converge (in energy norm) to a soliton as $t \rightarrow \infty$, are the solitons.

In conclusion, to establish the asymptotic stability, we need to weaken the notion of convergence. Indeed, using the weak convergence in the space $\mathcal{N} \mathcal{V}(\mathbb{R})$, Bahri [2] proved the asymptotic stability of solitons in the hydrodynamical framework.

Theorem $4.3([2])$. Let $c \in(-1,1) \backslash\{0\}$. There is $\alpha^{*}>0$ such that, if the initial condition $\mathfrak{v}^{0} \in \mathcal{N} \mathcal{V}(\mathbb{R})$ satisfies that $\left\|\mathfrak{v}^{0}-\mathfrak{v}_{c}\right\|_{H^{1} \times L^{2}}<\alpha^{*}$, then there exist a unique global associated solution $\mathfrak{v} \in \mathcal{C}^{0}(\mathbb{R}, \mathcal{N} \mathcal{V}(\mathbb{R}))$ to $(13), c^{*} \in(-1,1) \backslash\{0\}$ and $a \in \mathcal{C}^{1}(\mathbb{R}, \mathbb{R})$ such that, as $t \rightarrow \infty$,

$$
\mathfrak{v}(\cdot+a(t), t)) \rightarrow \mathfrak{v}_{c^{*}} \quad \text { in } H^{1}(\mathbb{R}) \times L^{2}(\mathbb{R}), \quad \text { and } \quad a^{\prime}(t) \rightarrow c^{*} .
$$

This theorem provides the weak convergence towards a soliton, but this long-time dynamics needs to take into account the geometric invariances of the problem, i.e. the translations. This is precisely the role of the parameter $a(t)$, whose derivative converges to the speed of the limit soliton $\mathfrak{v}_{c^{*}}$. In this fashion, the solution propagates with the same speed as the limit soliton, as $t$ goes to infinity, as expected.

The weak convergence in Theorem 4.3 can probably be improved. Indeed, Martel and Merle [81, 82] proved the asymptotic stability of solitons of the KdV equation, establishing a locally (strong) convergence in the energy space. It is possible that a similar result can be shown for the asymptotic stability of hydrodynamical solitons of the LL equation satisfy a similar, i.e. a strong convergence in a norm of the type $H^{1}([-R(t), R(t)]) \times L^{2}([-R(t), R(t)])$, where $R(t)$ is a linear function of time. 
The proof of Theorem 4.3 is based on an approach developed by Martel and Merle for the KdV equation [81, 82. Their strategy can be decomposed in three steps, that we would explain in our context, i.e. in the hydrodynamical setting. First, the orbital stability provided by Theorem 4.1 guarantees that a solution $\mathfrak{v}$, with initial condition $\mathfrak{v}^{0}$ close enough to a soliton $\mathfrak{v}_{c}$, remains in a neighborhood of the orbit of the soliton. In particular, the solution $\mathfrak{v}$ is bounded in the nonvanishing space $\mathcal{N} \mathcal{V}(\mathbb{R})$ for any $t \geq 0$. It is then possible to construct a sequence of times $\left(t_{n}\right)$, with $t_{n} \rightarrow \infty$, and a limit function $\mathfrak{v}_{*}^{0} \in \mathcal{N} \mathcal{V}(\mathbb{R})$, such that, up to a subsequence,

$$
\mathfrak{v}\left(\cdot, t_{n}\right) \rightarrow \mathfrak{v}_{*}^{0} \quad \text { in } \quad H^{1}(\mathbb{R}) \times L^{2}(\mathbb{R}),
$$

as $n \rightarrow \infty$. In addition, $\mathfrak{v}_{*}^{0}$ remains close to the orbit of the soliton $\mathfrak{v}_{c}$. Moreover, the solution $\mathfrak{v}_{*}$ to (H1d) with initial condition $\mathfrak{v}_{*}^{0}$ is global, and is also close to this orbit. We point out that is also necessary to introduce a modulation parameter due to the invariance by translation, but we will omit it for the sake of clarity.

We need to prove that the limit profile $\mathfrak{v}_{*}^{0}$, and the associated solution $\mathfrak{v}_{*}$, are indeed solitons. Thus, the second step is to study the regularity and decay properties of $\mathfrak{v}_{*}$. To this end, it is useful to establish the weak continuity of the flow of the hydrodynamical equation with respect to the initial condition, which implies that the solution $\mathfrak{v}$ converges to $\mathfrak{v}_{*}$, i.e. for any $t \in \mathbb{R}$ (fixed),

$$
\mathfrak{v}\left(\cdot, t_{n}+t\right) \rightarrow \mathfrak{v}_{*}(\cdot, t) \quad \text { in } H^{1}(\mathbb{R}) \times L^{2}(\mathbb{R}), \quad \text { as } n \rightarrow \infty .
$$

Using also a monotonicity formula for the momentum, from this convergence it is possible to deduce that $\mathfrak{v}_{*}$ is localized in space, uniformly in time, and that $\mathfrak{v}_{*}$ has an exponential decay in space, uniformly in time. Thus, using the Kato smoothing effect that gives regularizing properties of the Schrödinger-type equations, it follows that $\mathfrak{v}_{*}$ is of class $\mathcal{C}^{\infty}$ on $\mathbb{R} \times \mathbb{R}$, and that all its derivatives also decay in space, uniformly in time.

The third step is to show that in the neighborhood of a soliton, the only solutions to (H1d) having this behavior are the solitons. This rigidity property follows from a Liouville type theorem. The proof of this theorem requires another monotonicity formula, and it is the most difficult part of the argument. We refer to [2] for more details.

By refining the approach described above, Bahri [3] also established the asymptotic stability for initial data close to a sum of solitons, that are as usual well-prepared according to their speeds and have sufficiently separated initial positions. The proof of this theorem relies on the strategy developed by Martel, Merle and Tsai in [85] for the KdV equation. Let us also remark that the locally strong asymptotic stability result for multisolitons in [85] is stronger than the statement in [3] with $M=2$. Indeed, the proof in [85] is based on a monotonicity argument for the localized energy. It is an open problem if this kind of argument can be adapted to the study of the LL equation, or more generally, if it possible to get a locally strong asymptotic stability result.

In the higher dimensional case $N \geq 2$, most of the questions about solitons are still open. We refer to 33 and the references therein for more details.

\section{Self-similar solutions for the LLG equation}

In this section we will study the dissipative LLG equation (7). We will focus on the existence of self-similar solutions and provide their asymptotics in dimension $N=1$. We also analyze the qualitative and quantitative effect of the damping $\alpha$ on the dynamical behavior of these self-similar solutions. 
As we will see, these kinds of solutions do not belong to classical Sobolev spaces, and we cannot invoke the Cauchy theory developed in Section 2 to give a meaning to their stability. Therefore, we will provide a well-posedness result in a more general framework related to the BMO space to give some stability results. We point out that the proof of the well-posedness result uses the parabolic behavior of the equation in presence of damping, and cannot be applied for the pure dispersive equation (i.e. $\alpha=0$ ) analyzed in previous sections.

\subsection{Self-similar solutions}

A natural question, that has been proven relevant for understanding the global behavior of solutions and formation of singularities, is whether there exist solutions which are invariant under scalings of the equation. In the case of equation (7), it is straightforward to see that it is invariant under the following scaling: If $\boldsymbol{m}$ is a solution of $(7)$, then $\boldsymbol{m}_{\lambda}(x, t)=\boldsymbol{m}\left(\lambda x, \lambda^{2} t\right)$ is also a solution, for any $\lambda>0$. Associated with this invariance, a solution $\boldsymbol{m}$ of $(7)$ defined for $I=\mathbb{R}^{+}$or $I=\mathbb{R}^{-}$is called self-similar if it is invariant under rescaling, that is

$$
\boldsymbol{m}(x, t)=\boldsymbol{m}\left(\lambda x, \lambda^{2} t\right), \quad \forall \lambda>0, \quad \forall x \in \mathbb{R}^{N}, \quad \forall t \in I .
$$

Setting $T \in \mathbb{R}$ and performing a translation in time, this definition leads to two types of self-similar solutions: A forward self-similar solution, or expander, is a solution of the form $\boldsymbol{m}(x, t)=\boldsymbol{f}(x / \sqrt{t-T})$ for $(x, t) \in \mathbb{R}^{N} \times(T, \infty)$, and a backward self-similar solution, or shrinker, is a solution of the form $\boldsymbol{m}(x, t)=\boldsymbol{f}(x / \sqrt{T-t})$ for $(x, t) \in \mathbb{R}^{N} \times(-\infty, T)$, for certain profile $\boldsymbol{f}: \mathbb{R}^{N} \longrightarrow \mathbb{S}^{2}$. Expanders evolve from a singular value at time $T$, while shrinkers evolve towards a singular value at time $T$.

Self-similar solutions have brought a lot of attention in the study on nonlinear PDEs because they can provide some important information about the dynamics of the equation. While expanders are related to nonuniqueness phenomena, resolution of singularities and long time description of solutions, shrinkers are often related to phenomena of singularity formation (see e.g. [52, 42]). On the other hand, the construction and understanding of the dynamics and properties of self-similar solutions also provide an idea of which are the natural spaces to develop a well-posedness theory, that captures these very often physically relevant structures. Examples of equations for which self-similar solutions have been considered, and a substantial work around these types of solutions has been done, include among others the Navier-Stokes equation, semilinear parabolic equations, and geometric flows such as Yang-Mills, mean curvature flow and harmonic map flow. We refer to [66, 96] and the references therein for more details.

Most of the works in the literature related to the study of self-similar solutions to the LLG equation are confined to the heat flow for harmonic maps equation, i.e. $\alpha=1$. In this setting, the main works on the subject restrict the analysis to corotational maps taking values in $\mathbb{S}^{d}$, which reduces the analysis of (6) to the study of a second order real-valued ODE. Then tools such as the maximum principle or the shooting method can be used to show the existence of solutions. We refer to [44, 46, 50, 16, 19, 15, 48, for more details on such results for maps taking values in $\mathbb{S}^{d}$, with $d \geq 3$. Recently, Deruelle and Lamm [38] have studied the Cauchy problem for the harmonic map heat flow with initial data $\boldsymbol{m}^{0}: \mathbb{R}^{N} \rightarrow \mathbb{S}^{d}$, with $N \geq 3$ and $d \geq 2$, where $\boldsymbol{m}^{0}$ is Lipschitz 0-homogeneous function, homotopic to a constant, which implies the existence of expanders coming out of $\boldsymbol{m}^{0}$.

When $0<\alpha \leq 1$, we established the existence of self-similar expanders for the LLG equation in [60]. This result is a consequence of a well-possedness theorem for the LLG equation considering an initial data $\boldsymbol{m}^{0}: \mathbb{R}^{N} \rightarrow \mathbb{S}^{2}$ in the space BMO of functions of bounded mean oscillation. Notice that this result includes in particular the case of the harmonic map heat flow. We will explain more precisely this result in Section 5.3 . 
As seen before, in absence of damping $(\alpha=0)$, 7) reduces to the Schrödinger map equation (4), which is reversible in time, so that the notions of expanders and shrinkers coincide. For this equation, Germain, Shatah and Zeng [51] established the existence of ( $k$-equivariant) self-similar profiles $\boldsymbol{f}: \mathbb{R}^{2} \rightarrow \mathbb{S}^{2}$.

In the one-dimensional case, when $\alpha=0,(4)$ is closely related Localized Induction Approximation (LIA), and self-similar profiles $f: \mathbb{R} \rightarrow \mathbb{S}^{2}$ were obtained and analyzed in [62, 63, 74]. In the context of LIA, self-similar solutions constitute a family of smooth solutions that develop a singularity in the shape of a corner in finite time. For further work related to these solutions, including the study of the continuation of these solutions after the blow-up time and their stability, we refer to the reader to [8, 5]. At the level of the Schrödinger map equation, these self-similar solutions provide examples of smooth solutions that develop a jump singularity in finite time.

In this section we explain how to construct the family of expanders profiles for $\alpha \in[0,1]$, and provide their analytical study and we discuss the Cauchy problem associated with these solutions and their stability. Finally, we construct and analyze the family of shrinkers profiles.

\subsection{Expanders in dimension one}

We consider in this section equation (7) in dimension $N=1$, and $\alpha \in[0,1]$, in order to include both the damped and undamped cases. We seek self-similar solutions of the form

$$
\boldsymbol{m}(x, t)=\mathfrak{m}(x / \sqrt{t}), \quad x \in \mathbb{R}, t>0,
$$

and we will say that $\boldsymbol{m}$ is the profile of the solution $\boldsymbol{m}$. Observe that if $\boldsymbol{m}$ is a smooth solution to (7), it can be checked that $\mathfrak{m}$ solves the following system of ODEs

$$
\alpha \mathfrak{m}^{\prime \prime}+\alpha\left|\mathfrak{m}^{\prime}\right|^{2} \mathfrak{m}+\beta\left(\mathfrak{m} \times \mathfrak{m}^{\prime}\right)^{\prime}+\frac{x \mathfrak{m}^{\prime}}{2}=0, \quad \text { on } \mathbb{R},
$$

due to the fact that $\mathfrak{m}$ takes values in $\mathbb{S}^{2}$. Thus, we can give a weak formulation to this equation in the form $-\left(\mathcal{A}(\mathfrak{m}) \mathfrak{m}^{\prime}\right)^{\prime}=G\left(x, \mathfrak{m}, \mathfrak{m}^{\prime}\right)$, with

$$
\mathcal{A}(\boldsymbol{u})=\left(\begin{array}{ccc}
\alpha & -\beta u_{3} & \beta u_{2} \\
\beta u_{3} & \alpha & -\beta u_{1} \\
-\beta u_{2} & \beta u_{1} & \alpha,
\end{array}\right), G(x, \boldsymbol{u}, \boldsymbol{p})=\left(\begin{array}{c}
\alpha u_{1}|\boldsymbol{p}|^{2}-\frac{x p_{1}}{2} \\
\alpha u_{2}|\boldsymbol{p}|^{2}-\frac{x p_{2}}{2} \\
\alpha u_{3}|\boldsymbol{p}|^{2}-\frac{x p_{3}}{2}
\end{array}\right),
$$

where $\boldsymbol{u}=\left(u_{1}, u_{2}, u_{3}\right)$ and $\boldsymbol{p}=\left(p_{1}, p_{2}, p_{3}\right)$.

Therefore, if $\alpha>0$, the system is uniformly elliptic, since $\mathcal{A}(\boldsymbol{u}) \boldsymbol{\xi} \cdot \boldsymbol{\xi}=\alpha|\boldsymbol{\xi}|^{2}$, for all $\boldsymbol{\xi}, \boldsymbol{u} \in \mathbb{R}^{3}$, and we can then invoke the regularity theory for quasilinear elliptic systems, to verify that the solutions are smooth.

In the limit case $\alpha=0$, we can show directly that the solutions are also smooth. Most importantly, we have the following theorem that provides a rigidity result concerning the possible solutions to (32): The modulus of the gradient of any solution must be $c e^{-\alpha x^{2} / 4}$, for some $c \geq 0$.

Theorem 5.1 ([61]). Let $\alpha \in[0,1]$. Assume that $\mathfrak{m} \in H_{\text {loc }}^{1}\left(\mathbb{R} ; \mathbb{S}^{2}\right)$ is a weak solution to $(32)$. Then $\mathfrak{m}$ belongs to $\mathcal{C}^{\infty}\left(\mathbb{R} ; \mathbb{S}^{2}\right)$ and there exists $c \geq 0$ such that $\left|\mathfrak{m}^{\prime}(x)\right|=c e^{-\alpha x^{2} / 4}$, for all $x \in \mathbb{R}$.

In the limit cases $\alpha=1$ and $\alpha=0$, it is possible to find explicit solutions to (32), as we will see later on. However, this seems unlikely in the case $\alpha \in(0,1)$, and even the existence of such solutions is not clear. We proceed now to give a way of establishing the existence of solutions 
satisfying the condition $\left|\mathfrak{m}^{\prime}(x)\right|=c e^{-\alpha x^{2} / 4}$, for any $c>0$ and any $\alpha \in[0,1]$ (notice that the case $c=0$ corresponds to the trivial constant solution).

The idea is to look for $\mathfrak{m}$ as the tangent vector to a curve in $\mathbb{R}^{3}$, so we first recall some facts about curves in the space. Given $\mathfrak{m}: \mathbb{R} \rightarrow \mathbb{S}^{2}$ a smooth function, we can define the curve

$$
\boldsymbol{X}_{\mathfrak{m}}(x)=\int_{0}^{x} \mathfrak{m}(s) d s
$$

so that $\boldsymbol{X}_{\mathfrak{m}}$ is smooth, parametrized by arclenght, and its tangent vector is $\mathfrak{m}$. In addition, if $\left|\mathfrak{m}^{\prime}\right|$ does not vanish on $\mathbb{R}$, we can define the normal vector $\mathfrak{n}(x)=\mathfrak{m}^{\prime}(x) /\left|\mathfrak{m}^{\prime}(x)\right|$ and the binormal vector $\mathfrak{b}(x)=\mathfrak{m}(x) \times \mathfrak{n}(x)$. Moreover, we can define the curvature and torsion of $\boldsymbol{X}_{\mathfrak{m}}$ as $k(x)=\left|\mathfrak{m}^{\prime}(x)\right|$ and $\tau(x)=-\mathfrak{b}^{\prime}(x) \cdot \mathfrak{n}(x)$. Since $|\mathfrak{m}(x)|^{2}=1$, for all $x \in \mathbb{R}$, we have that $\mathfrak{m}(x) \cdot \mathfrak{n}(x)=0$, for all $x \in \mathbb{R}$, that the vectors $\{\mathfrak{m}, \mathfrak{n}, \mathfrak{b}\}$ are orthonormal and it is standard to check that they satisfy the Serret-Frenet system

$$
\mathfrak{m}^{\prime}=k \mathfrak{n}, \quad \mathfrak{n}^{\prime}=-k \mathfrak{m}+\tau \mathfrak{b}, \quad \mathfrak{b}^{\prime}=-\tau \mathfrak{n} .
$$

Let us apply this method to find a solution to (32). We define $\boldsymbol{X}_{\mathfrak{m}}$ as in (33), and we remark that equation 32 rewrites in terms of $\{\mathfrak{m}, \mathfrak{n}, \mathfrak{b}\}$ as

$$
-\frac{x}{2} k \mathfrak{n}=\beta\left(k^{\prime} \mathfrak{b}-\tau k \mathfrak{n}\right)-\alpha\left(-k^{\prime} \mathfrak{n}-k \tau \mathfrak{b}\right) .
$$

Therefore, from the orthogonality of the vectors $\mathfrak{n}$ and $\mathfrak{b}$, we conclude that the curvature and torsion of $\boldsymbol{X}_{\mathfrak{m}}$ are solutions of the equations $-x k=2 \alpha k^{\prime}-\beta \tau k$ and $\beta k^{\prime}+\alpha k \tau=0$, that is

$$
k(x)=c e^{-\alpha x^{2} / 4} \quad \text { and } \quad \tau(x)=\beta x / 2,
$$

for some $c \geq 0$. Of course, the fact that $k(x)=c e^{-\alpha x^{2} / 4}$ is in agreement with $\left|\mathfrak{m}^{\prime}(x)\right|=c e^{-\alpha x^{2} / 4}$.

Now, given $\alpha \in[0,1]$ and $c>0$, consider the Serret-Frenet system (34) with curvature and torsion function given by (35) and initial conditions $\mathfrak{m}(0)=\boldsymbol{e}_{1}, \mathfrak{n}(0)=\boldsymbol{e}_{2}, \mathfrak{b}(0)=\boldsymbol{e}_{3}$. Then, by standard ODE theory, there exists a unique global solution $\left\{\mathfrak{m}_{c, \alpha}, \mathfrak{n}_{c, \alpha}, \mathfrak{b}_{c, \alpha}\right\}$ in $\left(\mathcal{C}^{\infty}\left(\mathbb{R} ; \mathbb{S}^{2}\right)\right)^{3}$, and these vectors are orthonormal. Also, it is straightforward to verify that $\mathfrak{m}_{c, \alpha}$ is a solution to 32 satisfying $\left|\mathfrak{m}_{c, \alpha}^{\prime}(x)\right|=c e^{-\alpha x^{2} / 4}$.

Finally, using the uniqueness of the Cauchy-Lipschitz theorem and the Serret-Frenet system, it is simple to show the uniqueness of such solutions, up to rotations.

Theorem 5.2 (61]). The set of nonconstant solutions to $\left[32\right.$ is $\left\{\mathcal{R m}_{c, \alpha}: c>0, \mathcal{R} \in S O(3)\right\}$, where $S O(3)$ is the group of rotations about the origin preserving orientations.

The above proposition reduces the study of expanders to the understanding of the family of expanders associated with the profiles $\left\{\mathfrak{m}_{c, \alpha}\right\}_{c, \alpha}$. The next result summarizes the properties of these solutions.

Theorem $5.3([59])$. Let $\alpha \in[0,1], c \geq 0$ and $\mathfrak{m}_{c, \alpha}$ be the solution of the Serret-Frenet system constructed above. Let $\boldsymbol{m}_{c, \alpha}(x, t)=\mathfrak{m}_{c, \alpha}(x / \sqrt{t})$, for $(x, t) \in \mathbb{R} \times(0, \infty)$. Then the following statements hold.

(i) The function $\boldsymbol{m}_{c, \alpha}$ is a $\mathcal{C}^{\infty}$-solution of $(7)$ on $\mathbb{R} \times(0, \infty)$, with $\left|\partial_{x} \boldsymbol{m}_{c, \alpha}(x, t)\right|=\frac{c}{\sqrt{t}} e^{-\alpha x^{2} / 4 t}$.

(ii) There exists a unitary vector $\boldsymbol{A}_{c, \alpha}^{+}=\left(A_{j, c, \alpha}^{+}\right)_{j=1}^{3} \in \mathbb{S}^{2}$ such that $\boldsymbol{m}_{c, \alpha}(\cdot, t)$ converges pointwise to the initial condition

$$
\boldsymbol{m}_{c, \alpha}^{0}=\boldsymbol{A}_{c, \alpha}^{+} \chi_{\mathbb{R}^{+}}+\boldsymbol{A}_{c, \alpha}^{-} \chi_{\mathbb{R}^{-}}
$$

i.e.

$$
\lim _{t \rightarrow 0^{+}} \boldsymbol{m}_{c, \alpha}(x, t)=\boldsymbol{A}_{c, \alpha}^{+}, \text {if } x>0, \text { and } \lim _{t \rightarrow 0^{+}} \boldsymbol{m}_{c, \alpha}(x, t)=\boldsymbol{A}_{c, \alpha}^{-} \text {, if } x<0,
$$

where $\boldsymbol{A}_{c, \alpha}^{-}=\left(A_{1, c, \alpha}^{+},-A_{2, c, \alpha}^{+},-A_{3, c, \alpha}^{+}\right)$and $\chi_{E}$ is the characteristic function of the set $E$. 
(iii) Moreover, there exists a constant $C(c, \alpha, p)$ such that for all $t>0$ and all for all $p \in(1, \infty)$,

$$
\left\|\boldsymbol{m}_{c, \alpha}(\cdot, t)-\boldsymbol{m}_{c, \alpha}^{0}\right\|_{L^{p}(\mathbb{R})} \leq C(c, \alpha, p) t^{\frac{1}{2 p}} .
$$

The graphics in Figure 1 depict the profile $\mathfrak{m}_{c, \alpha}$ for fixed $c=0.8$ and the values of $\alpha=0.01$, $\alpha=0.2$, and $\alpha=0.4$. In particular, it can be observed how the convergence of $\mathfrak{m}_{c, \alpha}$ to $\boldsymbol{A}_{c, \alpha}^{ \pm}$is accelerated by the diffusion $\alpha$.

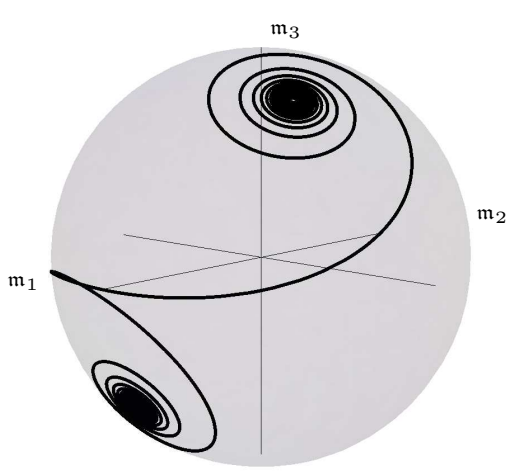

(a) $\alpha=0.01$

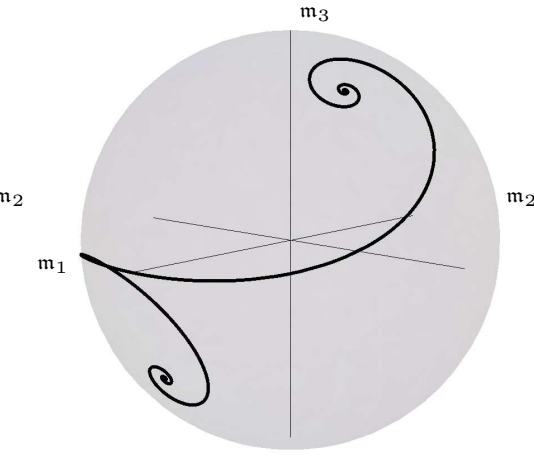

(b) $\alpha=0.2$

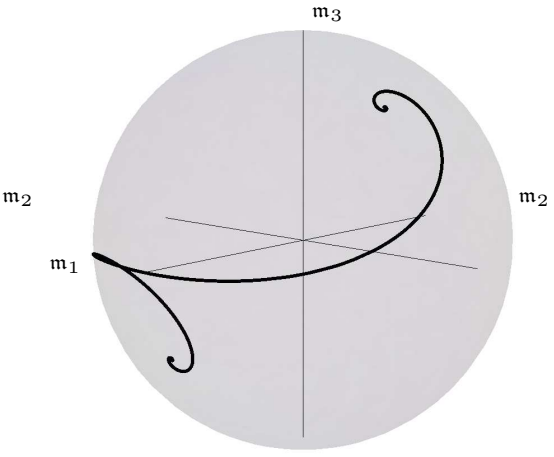

(c) $\alpha=0.4$

Figure 1: The profile $\mathfrak{m}_{c, \alpha}$ for $c=0.8$ and different values of $\alpha$.

Notice that the initial condition $\boldsymbol{m}_{c, \alpha}^{0}$ has a jump singularity at the point $x=0$ whenever the vectors $\boldsymbol{A}_{c, \alpha}^{+}$and $\boldsymbol{A}_{c, \alpha}^{-}$satisfy $\boldsymbol{A}_{c, \alpha}^{+} \neq \boldsymbol{A}_{c, \alpha}^{-}$. In this situation (and we will be able to prove analytically that this is the case, at least for certain ranges of the parameters $\alpha$ and $c$, see Proposition 5.5 below), Theorem 5.3 provides a family of global smooth solutions of (7) associated with a discontinuous singular initial data (jump-singularity).

As already mentioned, in the absence of damping $(\alpha=0)$, singular self-similar solutions of the Schrödinger map equation were previously obtained in [62, 74]. In this framework, Theorem 5.3 establishes the persistence of a jump singularity for self-similar solutions in the presence of dissipation.

When $\alpha=0$, the stability of the self-similar solutions was considered in a series of papers by Banica and Vega [5, 6, 7]. The stability in the case $\alpha>0$ is a natural question that we will discuss later.

Some further remarks on the results stated in Theorem 5.3 are in order. First, the energy is given by

$$
E_{\mathrm{LLG}}(t)=\frac{1}{2} \int_{-\infty}^{\infty}\left|\partial_{x} \boldsymbol{m}_{c, \alpha}(x, t)\right|^{2} d x=\frac{1}{2} \int_{-\infty}^{\infty}\left(\frac{c}{\sqrt{t}} e^{-\frac{\alpha x^{2}}{4 t}}\right)^{2} d x=c^{2} \sqrt{\frac{\pi}{\alpha t}}, \quad t>0 .
$$

It follows that the energy at the initial time $t=0$ is infinite, while it becomes finite for all positive times, showing the dissipation of energy in the system in the presence of damping.

Secondly, it is also important to remark that in the setting of Schrödinger equations, for fixed $\alpha \in[0,1]$ and $c>0$, the solution $\boldsymbol{m}_{c, \alpha}$ is associated through the Hasimoto transformation with the filament function [64], that is $u_{c, \alpha}(x, t)=\frac{c}{\sqrt{t}} e^{(-\alpha+i \beta) \frac{x^{2}}{4 t}}$, which solves

$$
i \partial_{t} u+(\beta-i \alpha) \partial_{x x} u+\frac{u}{2}\left(\beta|u|^{2}+2 \alpha \int_{0}^{x} \operatorname{Im}\left(\bar{u} \partial_{x} u\right)-A(t)\right)=0, \quad \text { with } \quad A(t)=\frac{\beta c^{2}}{t},
$$

with initial condition a Dirac delta function since $\lim _{t \rightarrow 0^{+}} u_{c, \alpha}(x, t)=2 c \sqrt{\pi(\alpha+i \beta)} \delta_{0}$. 
Therefore $u_{c, \alpha}$ is very rough at initial time and the standard arguments (e.g. a Picard iteration scheme based on Strichartz estimates and Sobolev-Bourgain spaces) cannot be applied, at least not straightforwardly, to study the local well-posedness of the initial value problem for the Schrödinger equation (37). The existence of solutions to equation (37) associated with an initial data proportional to a Dirac delta opens the question of developing a well-posedness theory for Schrödinger equations of the type considered here to include initial data of infinite energy. In the case $\alpha=0, A(t)=0$ and when the initial condition is proportional to the Dirac delta, Kenig, Ponce and Vega [67] proved that the Cauchy problem for (37) is ill-posed due to some oscillations. Moreover, even after removing these oscillations, Banica and Vega [5] showed that equation (37) (with $\alpha=0$ and $A(t)=c^{2} / t$ ) is still ill-posed. This question was also addressed by Vargas and Vega in [100] and Grünrock in [55] for other types of initial data of infinite energy (see also [4]), but we are not aware of any result in this setting when $\alpha>0$ (see [57] for related well-posedness results in the case $\alpha>0$ for initial data in Sobolev spaces of positive index).

\subsubsection{Asymptotics for the profile}

We want now to study the qualitative and quantitative effect of the damping $\alpha$ and the parameter $c$ on the dynamical behavior of the family $\left(\boldsymbol{m}_{c, \alpha}\right)_{c, \alpha}$ of self-similar solutions of (7) found in Theorem 5.3. Precisely, in an attempt to fully understand the regularization of the solution at positive times close to the initial time $t=0$, and to understand how the presence of damping affects the dynamical behavior of these self-similar solutions, we aim to give answers to the following questions: Can we obtain a more precise behavior of the solutions $\boldsymbol{m}_{c, \alpha}$ at positive times $t$ close to zero? Can we understand the limiting vectors $\boldsymbol{A}_{c, \alpha}^{ \pm}$in terms of the parameters $c$ and $\alpha$ ?

In order to address our first question, we observe that, due to the self-similar nature of these solutions, the behavior of the family of solutions $\boldsymbol{m}_{c, \alpha}$ at positive times close to the initial time $t=0$ is directly related to the study of the asymptotics of the associated profile $\mathfrak{m}_{c, \alpha}(x)$ for large values of $|x|$. In addition, the symmetries of $\mathfrak{m}_{c, \alpha}$ (see Theorem 5.4 below) allow to reduce ourselves to obtain the behavior of the profile as $x \rightarrow \infty$. The precise asymptotics of the profile is given in the following theorem.

Theorem 5.4 ([59]). Let $\alpha \in[0,1], c>0$. The components of $\mathfrak{m}_{c, \alpha}$ satisfy respectively that $\mathfrak{m}_{1, c, \alpha}$ is an even function, and $\mathfrak{m}_{j, c, \alpha}$ is an odd function for $j \in\{2,3\}$. In addition, for all $s \geq s_{0}=4 \sqrt{8+c^{2}}$,

$\mathfrak{m}_{c, \alpha}(s)=\boldsymbol{A}_{c, \alpha}^{+}-\frac{2 c}{s} \boldsymbol{B}_{c, \alpha}^{+} e^{-\alpha s^{2} / 4}\left(\alpha \sin \left(\phi_{c, \alpha}(s)\right)+\beta \cos \left(\phi_{c, \alpha}(s)\right)\right)-\frac{2 c^{2}}{s^{2}} \boldsymbol{A}_{c, \alpha}^{+} e^{-\alpha s^{2} / 2}+\mathcal{O}\left(\frac{e^{-\alpha s^{2} / 4}}{s^{3}}\right)$.

Here, $\sin \left(\phi_{c, \alpha}\right)$ and $\cos \left(\phi_{c, \alpha}\right)$ are understood acting on each of the components given by

$$
\phi_{j, c, \alpha}(s)=a_{j, \alpha, c}+\beta \int_{s_{0}^{2} / 4}^{s^{2} / 4} \sqrt{1+c^{2} \frac{e^{-2 \alpha \sigma}}{\sigma}} d \sigma, \quad j \in\{1,2,3\},
$$

for some constants $a_{1, \alpha, c}, a_{2, \alpha, c}, a_{1, \alpha, c} \in[0,2 \pi)$, and the vector $\boldsymbol{B}_{c, \alpha}^{+}$is given in terms of $\boldsymbol{A}_{c, \alpha}^{+}$by $\boldsymbol{B}_{c, \alpha}^{+}=\left(\left(1-\left(A_{1, c, \alpha}^{+}\right)^{2}\right)^{1 / 2},\left(1-\left(A_{2, c, \alpha}^{+}\right)^{2}\right)^{1 / 2},\left(1-\left(A_{3, c, \alpha}^{+}\right)^{2}\right)^{1 / 2}\right)$.

The convergence and rate of convergence of the solutions $\boldsymbol{m}_{c, \alpha}$ to $\boldsymbol{m}_{c, \alpha}^{0}$ established in Theorem 5.3 are simple consequences of the asymptotics in Theorem 5.4 . Also, similar asymptotics hold for the normal vector $\mathfrak{n}_{c, \alpha}$ and the binormal vector $\mathfrak{b}_{c, \alpha}$.

With regard to the asymptotics in Theorem 5.4, it is important to mention that the error depends only on $c$. More precisely, we use the notation $\mathcal{O}(f(s))$ to denote a function for which 
there exists a constant $C(c)>0$ depending on $c$, but but on $\alpha$, such that

$$
|\mathcal{O}(f(s))| \leq C(c)|f(s)|, \quad \text { for all } s \geq s_{0} .
$$

At first glance, one might think that the term $-2 c^{2} \boldsymbol{A}_{c, \alpha}^{+} e^{-\alpha s^{2} / 2} / s^{2}$ in the asymptotics could be included in the error term $\mathcal{O}\left(e^{-\alpha s^{2} / 4} / s^{3}\right)$. However, we cannot do this because in our notation the big- $\mathcal{O}$ must be independent of $\alpha$.

When $\alpha=1$ (so $\beta=0$ ), we can solve explicitly the Serret-Frenet system, to obtain

$$
\mathfrak{m}_{c, 1}(s)=(\cos (c \operatorname{Erf}(s)), \sin (c \operatorname{Erf}(s)), 0),
$$

for all $s \in \mathbb{R}$, where Erf is the non-normalized error function $\operatorname{Erf}(s)=\int_{0}^{s} e^{-\sigma^{2} / 4} d \sigma$. In particular, the limiting vectors in Theorem 5.4 are given by

$$
\boldsymbol{A}_{c, 1}^{ \pm}=(\cos (c \sqrt{\pi}), \pm \sin (c \sqrt{\pi}), 0), \quad \boldsymbol{B}_{c, 1}^{+}=(|\sin (c \sqrt{\pi})|,|\cos (c \sqrt{\pi})|, 1) .
$$

When $\alpha=0$, the solution of (34) can be solved explicitly in terms of parabolic cylinder functions or confluent hypergeometric functions (see [45]). Another analytical approach using Fourier analysis techniques has been taken in [62, leading to the asymptotics

$$
\mathfrak{m}_{c, 0}(s)=\boldsymbol{A}_{c, 0}^{+}-\frac{2 c}{s} \boldsymbol{B}_{c, 0}^{+} \sin \left(\psi_{c}\right)+\mathcal{O}\left(1 / s^{2}\right), \quad \text { with } \psi_{c}(s)=\frac{s^{2}}{4}+c^{2} \ln (s) .
$$

Moreover, $\boldsymbol{A}_{c, 0}^{+}$can be computed explicitly. On the other hand, when $\alpha=0$, the phase $\boldsymbol{\phi}_{c, \alpha}$ in (38) can be expanded as

$$
\phi_{j, c, 0}(s)=a_{j, c, \alpha}+\frac{s^{2}}{4}+c^{2} \ln (s)+C(c)+\mathcal{O}\left(1 / s^{2}\right) .
$$

Thus the asymptotics in Theorem 5.14 allows us to recover the logarithmic contribution in the oscillation in (41).

When $\alpha>0, \phi_{c, \alpha}$ behaves like

$$
\phi_{j, c, \alpha}(s)=a_{j, c, \alpha}+\frac{\beta s^{2}}{4}+C(\alpha, c)+\mathcal{O}\left(\frac{e^{-\alpha s^{2} / 2}}{\alpha s^{2}}\right),
$$

and there is no logarithmic correction in the oscillations in the presence of damping. Consequently, the phase function $\phi_{c, \alpha}$ captures the different nature of the oscillatory character of the solutions in both the absence and the presence of damping.

It can be seen that the terms $\boldsymbol{A}_{c, \alpha}^{+}, \boldsymbol{B}_{c, \alpha}^{+}, \boldsymbol{B}_{c, \alpha}^{+} \cdot \sin \left(a_{c, \alpha}\right), \boldsymbol{B}_{c, \alpha}^{+} \cdot \cos \left(a_{c, \alpha}\right)$ and the error term depend continuously on $\alpha \in[0,1]$. Therefore, the asymptotics in Theorem 5.14 shows how the profile $\mathfrak{m}_{c, \alpha}$ converges to $\mathfrak{m}_{c, 0}$ as $\alpha \rightarrow 0^{+}$and to $\mathfrak{m}_{c, 1}$ as $\alpha \rightarrow 1^{-}$. In particular, we recover the asymptotics in (41).

Finally, the amplitude of the leading order term controlling the wave-like behavior of the solution $\mathfrak{m}_{c, \alpha}(s)$ around $\boldsymbol{A}_{c, \alpha}^{ \pm}$for values of $s$ sufficiently large is of the order $c e^{-\alpha s^{2} / 4} / s$, from which one observes how the convergence of the solution to its limiting values $\boldsymbol{A}_{c, \alpha}^{ \pm}$is accelerated in the presence of damping in the system, as depicted in Figure 1 .

Let us discuss now some results answering the second of our questions. Bearing in mind that $\boldsymbol{A}_{c, \alpha}^{-}$is expressed in terms of the coordinates of $\boldsymbol{A}_{c, \alpha}^{+}$, we only need to focus on $\boldsymbol{A}_{c, \alpha}^{+}$. When $\alpha=1$ or $\alpha=0$, the vector $\boldsymbol{A}_{c, \alpha}^{+}$is explicitly given in terms of the parameter $c$. When $\alpha \in(0,1)$, we do not have explicit expressions for these vectors, however the following result establishes that the solutions $\boldsymbol{m}_{c, \alpha}$ of the LLG equation found in Theorem 5.3 are indeed associated with a discontinuous initial data at least for certain ranges of $\alpha$ and $c$. 


\section{Theorem $5.5([59])$.}

(i) Let $\alpha \in(0,1]$. There exists $c^{*}>0$ depending on $\alpha$ such that $\boldsymbol{A}_{c, \alpha}^{+} \neq \boldsymbol{A}_{c, \alpha}^{-}$, for all $c \in\left(0, c^{*}\right)$.

(ii) Let $c>0$. There exists $\alpha_{0}^{*}>0$ such that $\boldsymbol{A}_{c, \alpha}^{+} \neq \boldsymbol{A}_{c, \alpha}^{-}$, for all $\alpha \in\left(0, \alpha_{0}^{*}\right)$.

(iii) Let $c>0$, with $c \notin \mathbb{N} \sqrt{\pi}$. There exists $\alpha_{1}^{*} \in(0,1)$ such that $\boldsymbol{A}_{c, \alpha}^{+} \neq \boldsymbol{A}_{c, \alpha}^{-}$, for all $\alpha \in\left(\alpha_{1}^{*}, 1\right)$.

Remark 5.6. It can be checked that $\boldsymbol{A}_{c, 0}^{+} \neq \boldsymbol{A}_{c, 0}^{-}$for all $c>0$. Based on the numerical results in [59], we conjecture that $\boldsymbol{A}_{c, \alpha}^{+} \neq \boldsymbol{A}_{c, \alpha}^{-}$for all $\alpha \in(0,1)$ and $c>0$.

Concerning, the proof of the asymptotics of $\mathfrak{m}_{c, \alpha}$, a key tool is a classical change of variables from the differential geometry of curves that allows us to reduce the nine equations in the Serret-Frenet system into three complex-valued second order equations (see e.g. [75]). This change of variables is related to the stereographic projection; this approach was used in [62. In our case, the change of variables reduces the analysis of the solution $\left\{\mathfrak{m}_{c, \alpha}, \mathfrak{n}_{c, \alpha}, \mathfrak{b}_{c, \alpha}\right\}$ of the Serret-Frenet system to the study of three solutions to the second order differential equation

$$
f_{c, \alpha}^{\prime \prime}(s)+\frac{s}{2}(\alpha+i \beta) f_{c, \alpha}^{\prime}(s)+\frac{c^{2}}{4} e^{-\alpha s^{2} / 2} f_{c, \alpha}(s)=0,
$$

associated with three different initial conditions. The analysis of the solutions of (42) requires the control of certain integrals by exploiting their oscillatory character. This can be achieved by using repeated integration by parts, in the spirit of the method of stationary phase. We refer to [60] for more details of the proof.

\subsection{The Cauchy problem for LLG in BMO}

A natural question in the study of the stability properties of the family of solutions $\left(\boldsymbol{m}_{c, \alpha}\right)_{c>0}$ is whether it is possible to develop a well-posedness theory for the Cauchy problem for (7) in a functional framework that allows us to handle initial conditions of the type (36). In view of (36), such a framework should allow some "rough" functions (i.e. function spaces beyond the "classical" energy ones) and step functions.

In the case $\alpha>0$, global well-posedness results for (7) have been established in $N \geq 2$ by Melcher [88] and by Lin, Lai and Wang [79] for initial conditions with a smallness condition on the gradient in the $L^{N}\left(\mathbb{R}^{N}\right)$ and on the Morrey $M^{2,2}\left(\mathbb{R}^{N}\right)$-norm, respectively. Therefore, these results do not apply to the initial condition $\boldsymbol{m}_{c, \alpha}^{0}$. When $\alpha=1$, global well-posedness results for the heat flow for harmonic maps (6) have been obtained by Koch and Lamm [68] for an initial condition $L^{\infty}$-close to a point and improved to an initial data with small BMO semi-norm by Wang [102]. The ideas used in [68] and [102] rely on techniques introduced by Koch and Tataru 69] for the Navier-Stokes equation. Since $\boldsymbol{m}_{c, \alpha}^{0}$ has a small BMO semi-norm if $c$ is small, the results in [102] apply to the case $\alpha=1$.

In this subsection we explain the main results in [60] that allow us to adapt and extend the techniques developed in [68, 69, 102 to prove a global well-posedness result for (7) with $\alpha \in(0,1]$, for data $\boldsymbol{m}^{0}$ in $L^{\infty}\left(\mathbb{R}^{N} ; \mathbb{S}^{2}\right)$ with small BMO semi-norm. As an application of these results, we can establish the stability of the family of self-similar solutions $\left(\boldsymbol{m}_{c, \alpha}\right)_{c>0}$ and derive further properties for these solutions. In particular, we can prove the existence of multiple smooth solutions of (7) associated with the same initial condition, provided that $\alpha$ is close to one.

Our approach to study the Cauchy problem for (7) consists in analyzing the Cauchy problem for the associated dissipative quasilinear Schrödinger equation through the stereographic projection, and then "transferring" the results back to the original equation. To this end, we use the stereographic projection from the South Pole defined in (8). As mentioned in the introduction, if $\boldsymbol{m}$ is a smooth solution of (7) with $m_{3}>-1$, then its stereographic projection $u=\mathcal{P}(\boldsymbol{m})$ 
satisfies the quasilinear dissipative Schrödinger equation (DNLS). At least formally, the Duhamel formula gives the integral equation:

$$
u(x, t)=S_{\alpha}(t) u^{0}+\int_{0}^{t} S_{\alpha}(t-s) g(u)(s) d s, \quad \text { with } g(u)=-2 i(\beta-i \alpha) \frac{\bar{u}(\nabla u)^{2}}{1+|u|^{2}},
$$

where $u^{0}=u(\cdot, 0)$ corresponds to the initial condition, and $S_{\alpha}(t)$ is the dissipative Schrödinger semigroup (also called the complex Ginzburg-Landau semigroup) given by $S_{\alpha}(t) \phi=e^{(\alpha+i \beta) t \Delta} \phi$, i.e.

$$
\left(S_{\alpha}(t) \phi\right)(x)=\int_{\mathbb{R}^{N}} G_{\alpha}(x-y, t) \phi(y) d y, \quad \text { with } \quad G_{\alpha}(x, t)=\frac{e^{-\frac{|x|^{2}}{4(\alpha+i \beta) t}}}{(4 \pi(\alpha+i \beta) t)^{N / 2}} .
$$

One difficulty in studying (IDNLS) is to handle the term $g(u)$. We see that $|g(u)| \leq|\nabla u|^{2}$, so we need to control $|\nabla u|^{2}$. Koch and Taratu dealt with a similar problem when studying the well-posedness for the Navier-Stokes equation in [69]. Their approach was to introduce some new spaces related to BMO and $\mathrm{BMO}^{-1}$. Later, Koch and Lamm [68, and Wang [102] have adapted these spaces to study some geometric flows. Following these ideas, we define the Banach spaces

$$
\begin{aligned}
& X\left(\mathbb{R}^{N} \times \mathbb{R}^{+} ; F\right)=\left\{v: \mathbb{R}^{N} \times \mathbb{R}^{+} \rightarrow F: v, \nabla v \in L_{\mathrm{loc}}^{1}\left(\mathbb{R}^{N} \times \mathbb{R}^{+}\right),\|v\|_{X}<\infty\right\} \quad \text { and } \\
& Y\left(\mathbb{R}^{N} \times \mathbb{R}^{+} ; F\right)=\left\{v: \mathbb{R}^{N} \times \mathbb{R}^{+} \rightarrow F: v \in L_{\mathrm{loc}}^{1}\left(\mathbb{R}^{N} \times \mathbb{R}^{+}\right),\|v\|_{Y}<\infty\right\},
\end{aligned}
$$

where $\|v\|_{X}=\sup _{t>0}\|v\|_{L^{\infty}}+[v]_{X}$, with

$$
\begin{aligned}
& {[v]_{X}=\sup _{t>0} \sqrt{t}\|\nabla v\|_{L^{\infty}}+\sup _{\substack{x \in \mathbb{R}^{N} \\
r>0}}\left(\frac{1}{r^{N}} \int_{Q_{r}(x)}|\nabla v(y, t)|^{2} d t d y\right)^{\frac{1}{2}}, \quad \text { and }} \\
& \|v\|_{Y}=\sup _{t>0} t\|v\|_{L^{\infty}}+\sup _{\substack{x \in \mathbb{R}^{N} \\
r>0}} \frac{1}{r^{N}} \int_{Q_{r}(x)}|v(y, t)| d t d y .
\end{aligned}
$$

Here $Q_{r}(x)$ denotes the parabolic ball $Q_{r}(x)=B_{r}(x) \times\left[0, r^{2}\right]$ and $F$ is either $\mathbb{C}$ or $\mathbb{R}^{3}$. The absolute value stands for the complex absolute value if $F=\mathbb{C}$ and for the euclidean norm if $F=\mathbb{R}^{3}$. We denote with the same symbol the absolute value in $F$ and $F^{3}$.

The spaces $X$ and $Y$ are related to the spaces $\mathrm{BMO}\left(\mathbb{R}^{N}\right)$ and $\mathrm{BMO}^{-1}\left(\mathbb{R}^{N}\right)$ and are welladapted to study problems involving the heat semigroup $S_{1}(t)=e^{t \Delta}$. In order to establish the properties of the semigroup $S_{\alpha}(t)$ with $\alpha \in(0,1]$, we introduce the spaces $\mathrm{BMO}_{\alpha}\left(\mathbb{R}^{N}\right)$ and $\mathrm{BMO}_{\alpha}^{-1}\left(\mathbb{R}^{N}\right)$ as the space of distributions $f \in S^{\prime}\left(\mathbb{R}^{N} ; F\right)$ such that the semi-norm and norm given respectively by

$$
[f]_{B M O_{\alpha}}=\sup _{\substack{x \in \mathbb{R}^{N} \\ r>0}}\left(\frac{1}{r^{N}} \int_{Q_{r}(x)}\left|\nabla S_{\alpha}(t) f\right|^{2}\right)^{\frac{1}{2}}, \quad\|f\|_{B M O_{\alpha}^{-1}}=\sup _{\substack{x \in \mathbb{R}^{N} \\ r>0}}\left(\frac{1}{r^{N}} \int_{Q_{r}(x)}\left|S_{\alpha}(t) f\right|^{2}\right)^{\frac{1}{2}},
$$

are finite.

On the one hand, the Carleson measure characterization of BMO functions (see 95, Chapter 4] and [77, Chapter 10]) yields that for fixed $\alpha \in(0,1], \mathrm{BMO}_{\alpha}\left(\mathbb{R}^{N}\right)$ coincides with the classical $\operatorname{BMO}\left(\mathbb{R}^{N}\right)$ space, that is for all $\alpha \in(0,1]$ there exists a constant $\Lambda>0$ depending only on $\alpha$ and $N$ such that $\Lambda[f]_{B M O} \leq[f]_{B M O_{\alpha}} \leq \Lambda^{-1}[f]_{B M O}$. On the other hand, Koch and Tataru proved in [69] that $\mathrm{BMO}^{-1}$ (or equivalently $\mathrm{BMO}_{1}^{-1}$, using our notation) can be characterized as the space of derivatives of functions in BMO. A straightforward generalization of their argument shows that the same result holds for $\mathrm{BMO}_{\alpha}^{-1}$. Hence, using the Carleson measure characterization theorem, 
we conclude that $\mathrm{BMO}_{\alpha}^{-1}$ coincides with the space $\mathrm{BMO}^{-1}$ and that there exists a constant $\tilde{\Lambda}>0$, depending only on $\alpha$ and $N$, such that $\tilde{\Lambda}\|f\|_{B M O^{-1}} \leq\|f\|_{B M O_{\alpha}^{-1}} \leq \tilde{\Lambda}^{-1}\|f\|_{B M O^{-1}}$.

The above remarks allow us to use several of the estimates proved in [68, 69, 102] in the case $\alpha=1$, to study the integral equation (IDNLS by using a fixed-point approach. Finally, this leads to the next result that provides the global well-posedness of the Cauchy problem for IDNLS with small initial data in $\operatorname{BMO}\left(\mathbb{R}^{N}\right)$.

Theorem $5.7([60])$. Let $\alpha \in(0,1]$. There exist constants $C, K \geq 1$ such that for every $L \geq 0$, $\varepsilon>0$, and $\rho>0$ satisfying

$$
8 C(\rho+\varepsilon)^{2} \leq \rho
$$

if $u^{0} \in L^{\infty}\left(\mathbb{R}^{N} ; \mathbb{C}\right)$, with

$$
\left\|u^{0}\right\|_{L^{\infty}} \leq L \quad \text { and } \quad\left[u^{0}\right]_{B M O} \leq \varepsilon
$$

then there exists a unique solution $u \in X\left(\mathbb{R}^{N} \times \mathbb{R}^{+} ; \mathbb{C}\right)$ to IDNLS such that

$$
[u]_{X} \leq K(\rho+\varepsilon) .
$$

Moreover, $u \in \mathcal{C}^{\infty}\left(\mathbb{R}^{N} \times \mathbb{R}^{+}\right)$, (DNLS) holds pointwise, $\sup _{t>0}\|u\|_{L^{\infty}} \leq K(\rho+L)$ and $u(\cdot, t) \rightarrow u^{0}$, as $t \rightarrow 0^{+}$, as tempered distributions.

In addition, assume that $u$ and $v$ are respectively solutions to (IDNLS) fulfilling (45) with initial conditions $u^{0}$ and $v^{0}$ satisfying (44). Then $\|u-v\|_{X} \leq 6 K\left\|u^{0}-v^{0}\right\|_{L^{\infty}}$.

Although condition (43) appears naturally from the fixed-point used in the proof, it may not be so clear at first glance. To better understand it, let us define for $C>0$

$$
\mathcal{S}(C)=\left\{(\rho, \varepsilon) \in \mathbb{R}^{+} \times \mathbb{R}^{+}: C(\rho+\varepsilon)^{2} \leq \rho\right\} .
$$

We see that if $(\rho, \varepsilon) \in \mathcal{S}(C)$, then $\rho, \varepsilon>0$ and $\varepsilon \leq \frac{\sqrt{\rho}}{\sqrt{C}}-\rho$. Therefore, the set $\mathcal{S}(C)$ is non-empty and bounded. The shape of this set is depicted in Figure 2. In particular, we infer that if $(\rho, \varepsilon) \in \mathcal{S}(C)$, then $\rho \leq \frac{1}{C}$ and $\varepsilon \leq \frac{1}{4 C}$. In addition, if $\tilde{C} \geq C$, then $\mathcal{S}(\tilde{C}) \subseteq \mathcal{S}(C)$.

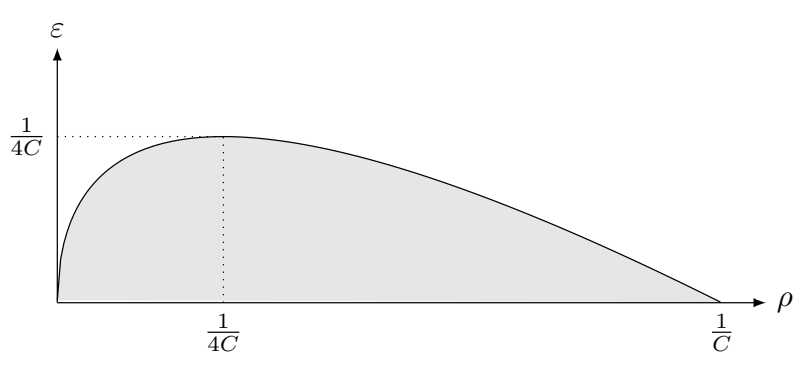

Figure 2: The shape of the set $\mathcal{S}(C)$.

Moreover, taking $\rho=1 /(32 C)$, Theorem 5.7 asserts that for fixed $\alpha \in(0,1]$, we can take for instance $\varepsilon=1 /(32 C)$ (that depends on $\alpha$ and $N$, but not on the $L^{\infty}$-norm of the initial data) such that for any given initial condition $u^{0} \in L^{\infty}\left(\mathbb{R}^{N}\right)$ with $\left[u^{0}\right]_{B M O} \leq \varepsilon$, there exists a global (smooth) solution $u \in X\left(\mathbb{R}^{N} \times \mathbb{R}^{+} ; \mathbb{C}\right)$ of $\left(\right.$ DNLS). Notice that $u^{0}$ is allowed to have a large $L^{\infty}$-norm as long as $\left[u^{0}\right]_{B M O}$ is sufficiently small; this is a weaker requirement that asking for the $L^{\infty}$-norm of $u^{0}$ to be sufficiently small, since $[f]_{B M O} \leq 2\|f\|_{L^{\infty}}$, for all $f \in L^{\infty}\left(\mathbb{R}^{N}\right)$.

We remark that the smallness condition in 45 is necessary for the uniqueness of the solution. As we will see in Theorem 5.12, at least in dimension one, it is possible to construct multiple solutions of (IDNLS) in $X\left(\mathbb{R}^{N} \times \mathbb{R}^{+} ; \mathbb{C}\right)$, if $\alpha$ is close enough to 1 . 
By using the inverse of the stereographic projection $\mathcal{P}^{-1}: \mathbb{C} \rightarrow \mathbb{S}^{2} \backslash\{0,0,-1\}$, that is explicitly given by $\boldsymbol{m}=\left(m_{1}, m_{2}, m_{3}\right)=\mathcal{P}^{-1}(u)$, with

$$
m_{1}=\frac{2 \operatorname{Re} u}{1+|u|^{2}}, \quad m_{2}=\frac{2 \operatorname{Im} u}{1+|u|^{2}}, \quad m_{3}=\frac{1-|u|^{2}}{1+|u|^{2}},
$$

we can deduce from Theorem 5.7 a global well-posedness result for (7). Moreover, the choice of the South Pole is of course arbitrary. By using the invariance of (7) under rotations, we have the existence of solutions provided that the essential range of the initial condition $\boldsymbol{m}^{0}$ is far from an arbitrary point $\boldsymbol{Q} \in \mathbb{S}^{2}$.

Theorem $5.8([60])$. Let $\alpha \in(0,1]$. There exist constants $C \geq 1$ and $K \geq 4$, such that if $\delta \in(0,2], \varepsilon_{0}, \rho>0, \delta \in(0,2], \varepsilon_{0}>0$ and $\rho>0$ satisfy

$$
8 K^{4} C \delta^{-4}\left(\rho+8 \delta^{-2} \varepsilon_{0}\right)^{2} \leq \rho
$$

the following holds. Given any $\boldsymbol{m}^{0}=\left(m_{1}^{0}, m_{2}^{0}, m_{3}^{0}\right) \in L^{\infty}\left(\mathbb{R}^{N} ; \mathbb{S}^{2}\right)$ and any $\boldsymbol{Q} \in \mathbb{S}^{2}$ satisfying

$$
\inf _{\mathbb{R}^{N}}\left|\boldsymbol{m}^{0}-\boldsymbol{Q}\right|^{2} \geq 2 \delta \quad \text { and } \quad\left[\boldsymbol{m}^{0}\right]_{B M O} \leq \varepsilon_{0},
$$

there exists a unique smooth solution $\boldsymbol{m} \in X\left(\mathbb{R}^{N} \times \mathbb{R}^{+} ; \mathbb{S}^{2}\right)$ of $(7)$ with initial condition $\boldsymbol{m}^{0}$ such that

$$
\inf _{\substack{x \in \mathbb{R}^{N} \\ t>0}}|\boldsymbol{m}(x, t)-\boldsymbol{Q}|^{2} \geq \frac{4}{1+K^{2}\left(\rho+\delta^{-1}\right)^{2}} \quad \text { and } \quad[\boldsymbol{m}]_{X} \leq 4 K\left(\rho+8 \delta^{-2} \varepsilon_{0}\right) .
$$

We point out that the results are valid only for $\alpha>0$. If we let $\alpha \rightarrow 0^{+}$, then the estimates blow up. Indeed, the proofs rely on the exponential decay of the semigroup $e^{(\alpha+i \beta) t \Delta}$, so that these techniques cannot be generalized (at least not straightforwardly) to cover the critical case $\alpha=0$. In particular, we cannot recover the stability results for the self-similar solutions in the case of Schrödinger maps proved by Banica and Vega in [5, 6, 7].

As mentioned before, in [79] and [88] some global well-posedness results for (7) with $\alpha \in(0,1]$ were proved for initial conditions with small gradient in $L^{N}\left(\mathbb{R}^{N}\right)$ and $M^{2,2}\left(\mathbb{R}^{N}\right)$, respectively. In view of the embeddings

$$
L^{N}\left(\mathbb{R}^{N}\right) \subset M^{2,2}\left(\mathbb{R}^{N}\right) \subset B M O^{-1}\left(\mathbb{R}^{N}\right),
$$

for $N \geq 2$, Theorem 5.8 can be seen as generalization of these results since it covers the case of less regular initial conditions. The arguments in [79, 88] are based on the method of moving frames that produces a covariant complex Ginzburg-Landau equation.

The existence and uniqueness results given by Theorem 5.8 require the initial condition to be small in the BMO semi-norm. Without this condition, the solution could develop a singularity in finite time. In fact, in dimensions $N=3,4$, Ding and Wang [39] proved that for some smooth initial conditions with small energy, the associated solutions of (7) blow up in finite time.

Another consequence of Theorem 5.8 is the existence of self-similar solutions of expander type in $\mathbb{R}^{N}$, in any dimension $N \geq 1$, i.e. a solution $\boldsymbol{m}$ of the form $\boldsymbol{m}(x, t)=\boldsymbol{f}(x / \sqrt{t})$, for some profile $\boldsymbol{f}: \mathbb{R}^{N} \rightarrow \mathbb{S}^{2}$. In particular, we have the relation $\boldsymbol{f}(y)=\boldsymbol{m}(y, 1)$, for all $y \in \mathbb{R}^{N}$. From the scaling (31), we see that, at least formally, a necessary condition for the existence of a self-similar solution is that initial condition $\boldsymbol{m}^{0}$ be homogeneous of degree 0 , i.e. $\boldsymbol{m}^{0}(\lambda x)=\boldsymbol{m}^{0}(x)$, for all $\lambda>0$. Since the norm in $X\left(\mathbb{R}^{N} \times \mathbb{R}^{+} ; \mathbb{R}^{3}\right)$ is invariant under this scaling, Theorem 5.8 yields the following result concerning the existence of self-similar solutions.

Corollary 5.9. With the same notations and hypotheses as in Theorem 5.8, assume also that $\boldsymbol{m}^{0}$ is homogeneous of degree zero. Then the solution $\boldsymbol{m}$ of (7) provided by Theorem 5.8 is forward self-similar. In particular there exists a smooth profile $\boldsymbol{f}: \mathbb{R}^{N} \rightarrow \mathbb{S}^{2}$ such that $\boldsymbol{m}(x, t)=\boldsymbol{f}(x / \sqrt{t})$, for all $x \in \mathbb{R}^{N}$ and $t>0$. 
Other authors have considered expanders for the harmonic map flow (6) in different settings. Actually, equation (6) can be generalized for maps $\boldsymbol{m}: \mathcal{M} \times \mathbb{R}^{+} \rightarrow \mathcal{N}$, with $\mathcal{M}$ and $\mathcal{N}$ Riemannian manifolds. Biernat and Bizoń [15] established results when $\mathcal{M}=\mathcal{N}=\mathbb{S}^{d}$ and $3 \leq d \leq 6$. Also, Germain and Rupflin [50] have investigated the case $\mathcal{M}=\mathbb{R}^{d}$ and $\mathcal{N}=\mathbb{S}^{d}$, in $d \geq 3$. In both works the analysis is done only for equivariant solutions and does not cover the case $\mathcal{M}=\mathbb{R}^{N}$ and $\mathcal{N}=\mathbb{S}^{2}$.

\subsection{LLG with a jump initial data}

We want now to apply the well-posedness result to the self-similar solutions $\boldsymbol{m}_{c, \alpha}$ with initial conditions $\boldsymbol{m}_{c, \alpha}^{0}=\boldsymbol{A}_{c, \alpha}^{+} \chi_{\mathbb{R}^{+}}+\boldsymbol{A}_{c, \alpha}^{-} \chi_{\mathbb{R}^{-}}$. Let us remark that the first term in the definition of $[\boldsymbol{v}]_{X}$ allows us to capture a blow-up rate of $1 / \sqrt{t}$ for $\|\nabla v(t)\|_{L^{\infty}}$, as $t \rightarrow 0^{+}$. This is exactly the blow-up rate for the self-similar solutions $\boldsymbol{m}_{c, \alpha}$. The integral term in the semi-norm $[\cdot]_{X}$ is also well-adapted to these solutions. Indeed, for any $\alpha \in(0,1]$ and $c \geq 0$, we have

$$
\left[\boldsymbol{m}_{c, \alpha}^{0}\right]_{B M O} \leq 2 c \sqrt{2 \pi} / \sqrt{\alpha} \quad \text { and } \quad\left[\boldsymbol{m}_{c, \alpha}\right]_{X} \leq 4 c / \alpha^{\frac{1}{4}} .
$$

Let us start by considering a more general problem: the LLG equation, in dimension one, with a jump initial data given by $\boldsymbol{m}_{\boldsymbol{A}^{ \pm}}^{0}=\boldsymbol{A}^{+} \chi_{\mathbb{R}^{+}}+\boldsymbol{A}^{-} \chi_{\mathbb{R}^{-}}$, where $\boldsymbol{A}^{ \pm}$are two given unitary vectors in $\mathbb{S}^{2}$. The smallness condition in the BMO semi-norm of $\boldsymbol{m}_{\boldsymbol{A}^{ \pm}}^{0}$ is equivalent to the smallness of the angle between $\boldsymbol{A}^{+}$and $\boldsymbol{A}^{-}$. From Theorem 5.8 we can deduce that the solution associated with $\boldsymbol{m}_{\boldsymbol{A}^{ \pm}}^{0}$ is a rotation of a self-similar solution $\boldsymbol{m}_{c, \alpha}$ for an appropriate value of $c$. Precisely,

Theorem 5.10 ([60]). Let $\alpha \in(0,1]$. There exist $L_{1}, L_{2}>0, \delta^{*} \in(-1,0)$ and $\vartheta^{*}>0$ such that the following holds. Let $\boldsymbol{A}^{+}, \boldsymbol{A}^{-} \in \mathbb{S}^{2}$ and let $\vartheta$ be the angle between them. If $0<\vartheta \leq \vartheta^{*}$, then there exists a solution $\boldsymbol{m}$ of (7) with initial condition $\boldsymbol{m}_{\boldsymbol{A} \pm}^{0}$. Moreover, there exists $0<c<\frac{\sqrt{\alpha}}{2 \sqrt{\pi}}$, such that $\boldsymbol{m}$ coincides up to a rotation with the self-similar solution $\boldsymbol{m}_{c, \alpha}$, i.e. there exists $\mathcal{R} \in S O(3)$, depending only on $\boldsymbol{A}^{+}, \boldsymbol{A}^{-}, \alpha$ and $c$, such that $\boldsymbol{m}=\mathcal{R} \boldsymbol{m}_{c, \alpha}$, and $\boldsymbol{m}$ is the unique solution satisfying

$$
\inf _{\substack{x \in \mathbb{R} \\ t>0}} m_{3}(x, t) \geq \delta^{*} \quad \text { and } \quad[\boldsymbol{m}]_{X} \leq L_{1}+L_{2} c .
$$

A second consequence of Theorem 5.8 concerns the stability of the self-similar solutions. Indeed, from the dependence of the solution with respect to the initial data in this theorem and the estimates in (46), we obtain the following result: For any given $\boldsymbol{m}^{0} \in \mathbb{S}^{2}$ close enough to $\boldsymbol{m}_{\boldsymbol{A}^{ \pm}}^{0}$, the solution $\boldsymbol{m}$ of $(7)$ associated with $\boldsymbol{m}^{0}$ given by Theorem 5.8 must remain close to a rotation of a self-similar solution $\boldsymbol{m}_{c, \alpha}$, for some $c>0$. In particular, $\boldsymbol{m}$ remains close to a self-similar solution. The precise statement is provided in the following theorem.

Theorem $5.11([60])$. Let $\alpha \in(0,1]$. There exist constants $L_{1}, L_{2}, L_{3}>0, \delta^{*} \in(-1,0), \vartheta^{*}>0$ such that the following holds. Let $\boldsymbol{A}^{+}, \boldsymbol{A}^{-} \in \mathbb{S}^{2}$ with angle $\vartheta$ between them. If $0<\vartheta \leq \vartheta^{*}$, then there is $c>0$ such that for every $\boldsymbol{m}^{0}$ satisfying $\left\|\boldsymbol{m}^{0}-\boldsymbol{m}_{\boldsymbol{A}^{ \pm}}^{0}\right\|_{L^{\infty}} \leq \frac{c \sqrt{\pi}}{2 \sqrt{\alpha}}$, there exists $\mathcal{R} \in S O(3)$, depending only on $\boldsymbol{A}^{+}, \boldsymbol{A}^{-}, \alpha$ and $c$, such that there is a unique global smooth solution $\boldsymbol{m}$ of (7) with initial condition $\boldsymbol{m}^{0}$ that satisfies

$$
\inf _{\substack{x \in \mathbb{R} \\ t>0}}(\mathcal{R} \boldsymbol{m})_{3}(x, t) \geq \delta^{*} \quad \text { and } \quad[\boldsymbol{m}]_{X} \leq L_{1}+L_{2} c .
$$

Moreover, $\left\|\boldsymbol{m}-\mathcal{R} \boldsymbol{m}_{c, \alpha}\right\|_{X} \leq L_{3}\left\|\boldsymbol{m}^{0}-\boldsymbol{m}_{\boldsymbol{A}^{ \pm}}^{0}\right\|_{L^{\infty}}$. In particular,

$$
\left\|\partial_{x} \boldsymbol{m}-\partial_{x} \mathcal{R} \boldsymbol{m}_{c, \alpha}\right\|_{L^{\infty}} \leq \frac{L_{3}}{\sqrt{t}}\left\|\boldsymbol{m}^{0}-\boldsymbol{m}_{\boldsymbol{A}^{ \pm}}^{0}\right\|_{L^{\infty}}, \quad \text { for all } t>0 .
$$


Let us now discuss the multiplicity of solutions with initial condition $\boldsymbol{m}_{\boldsymbol{A}^{ \pm}}^{0}$. As seen before, when $\alpha=1$, the self-similar solutions are explicitly given by $(39)$ and limit vectors $\vec{A}_{c, 1}^{ \pm}$given in (40).

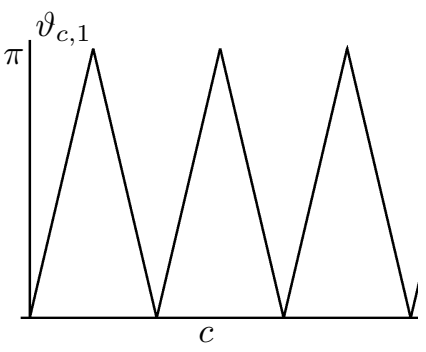

Figure 3: The angle $\vartheta_{c, \alpha}$ as a function of $c$ for $\alpha=1$.

Figure 3 shows that there are infinite values of $c$ that allow to reach any angle in $[0, \pi]$. Therefore, using the invariance of (7) under rotations, in the case when $\alpha=1$, one can easily prove the existence of multiple solutions associated with a given initial data of the form $\boldsymbol{m}_{\boldsymbol{A}^{ \pm}}^{0}$ for any given vectors $\boldsymbol{A}^{ \pm} \in \mathbb{S}^{2}$. In the case that $\alpha$ is close enough to 1, we can use a continuity argument to prove that we still have multiple solutions. More precisely, we can establish that for any given initial data of the form $\boldsymbol{m}_{\boldsymbol{A}^{ \pm}}^{0}$, with angle between $\boldsymbol{A}^{+}$and $\boldsymbol{A}^{-}$in the interval $(0, \pi)$, if $\alpha$ is sufficiently close to one, then there exist at least $k$-distinct solutions of (7) associated with the same initial condition, for any $k \in \mathbb{N}$. In other words, given any angle $\vartheta \in(0, \pi)$ between two $\boldsymbol{A}^{+}$and $\boldsymbol{A}^{-}$, we can generate any number of distinct solutions by considering values of $\alpha$ sufficiently close to 1 . Precisely,

Theorem $5.12([60])$. Let $k \in \mathbb{N}, \boldsymbol{A}^{+}, \boldsymbol{A}^{-} \in \mathbb{S}^{2}$ and let $\vartheta$ be the angle between $\boldsymbol{A}^{+}$and $\boldsymbol{A}^{-}$. If $\vartheta \in(0, \pi)$, then there exists $\alpha_{k} \in(0,1)$ such that for every $\alpha \in\left[\alpha_{k}, 1\right]$ there are at least $k$ distinct smooth self-similar solutions $\left\{\boldsymbol{m}_{j}\right\}_{j=1}^{k}$ in $X\left(\mathbb{R} \times \mathbb{R}^{+} ; \mathbb{S}^{2}\right)$ of (7) with initial condition $\boldsymbol{m}_{\boldsymbol{A}^{ \pm}}^{0}$. These solutions are characterized by a strictly increasing sequence of values $\left\{c_{j}\right\}_{j=1}^{k}$, with $c_{k} \rightarrow \infty$ as $k \rightarrow \infty$, such that $\boldsymbol{m}_{j}=\mathcal{R}_{j} \boldsymbol{m}_{c_{j}, \alpha}$, where $\mathcal{R}_{j} \in S O(3)$. In particular

$$
\sqrt{t}\left\|\partial_{x} \boldsymbol{m}_{j}(\cdot, t)\right\|_{L^{\infty}(\mathbb{R})}=c_{j}, \quad \text { for all } t>0 .
$$

Furthermore, if $\alpha=1$ and $\vartheta \in[0, \pi]$, then there is an infinite number of distinct smooth self-similar solutions $\left\{\boldsymbol{m}_{j}\right\}_{j \geq 1}$ in $X\left(\mathbb{R} \times \mathbb{R}^{+} ; \mathbb{S}^{2}\right)$ of $(7)$ with initial condition $\boldsymbol{m}_{\boldsymbol{A}^{ \pm}}^{0}$.

It is important to remark that in particular Theorem 5.12 asserts that when $\alpha=1$, given $\boldsymbol{A}^{+}, \boldsymbol{A}^{-} \in \mathbb{S}^{2}$ such that $\boldsymbol{A}^{+}=\boldsymbol{A}^{-}$, there exists an infinite number of distinct solutions $\left\{\boldsymbol{m}_{j}\right\}_{j \geq 1}$ in $X\left(\mathbb{R} \times \mathbb{R}^{+} ; \mathbb{S}^{2}\right)$ of $(7)$ with initial condition $\boldsymbol{m}_{\boldsymbol{A}^{ \pm}}^{0}$ such that $\left[\boldsymbol{m}_{\boldsymbol{A}^{ \pm}}^{0}\right]_{B M O}=0$. This particular case shows that a condition on the size of $X$-norm of the solution in Theorem 5.8 is necessary for the uniqueness of solution. We recall that for finite energy solutions of (6), there are several nonuniqueness results based on Coron's technique [30] in dimension $N=3$. Alouges and Soyeur [1] successfully adapted this idea to prove the existence of multiple solutions of (7), with $\alpha>0$, for maps $\boldsymbol{m}: \Omega \longrightarrow \mathbb{S}^{2}$, with $\Omega$ a bounded regular domain of $\mathbb{R}^{3}$. In our case, since $\left\{c_{j}\right\}_{j=1}^{k}$ is strictly increasing, we have at least $k$ different smooth solutions. Notice also that the identity (47) implies that the $X$-norm of the solution is large as $j \rightarrow \infty$.

\subsection{Shrinkers}

We end this note by discussing the backward self-similar solutions to (7), i.e. the shrinker solutions of the form $\boldsymbol{m}(x, t)=\boldsymbol{f}\left(\frac{x}{\sqrt{T-t}}\right)$, for $x \in \mathbb{R}$ and $t \in(-\infty, T)$. As in Section 5.2 , we can reduce 
our problem to the study of the ODE

$$
\alpha \boldsymbol{f}^{\prime \prime}+\alpha\left|\boldsymbol{f}^{\prime}\right|^{2} \boldsymbol{f}+\beta\left(\boldsymbol{f} \times \boldsymbol{f}^{\prime}\right)^{\prime}-\frac{x \boldsymbol{f}^{\prime}}{2}=0, \quad \text { on } \mathbb{R},
$$

which is the same equation that we obtained for the expanders, except for the minus sign in the last term. Following similar arguments, we get

Theorem 5.13 ([61]). Let $\alpha \in(0,1]$. Assume that $\boldsymbol{f} \in H_{\mathrm{loc}}^{1}\left(\mathbb{R} ; \mathbb{S}^{2}\right)$ is a weak solution to 48 . Then $\boldsymbol{f}$ belongs to $\mathcal{C}^{\infty}\left(\mathbb{R} ; \mathbb{S}^{2}\right)$ and there exists $c \geq 0$ such that $\left|\boldsymbol{f}^{\prime}(x)\right|=c e^{\alpha x^{2} / 4}$, for all $x \in \mathbb{R}$. Moreover, the set of nonconstant solutions to (48) is $\left\{\mathcal{R} \boldsymbol{f}_{c, \alpha}: c>0, \mathcal{R} \in S O(3)\right\}$, where is $\boldsymbol{f}_{c, \alpha}$ is given by the solution $\{\boldsymbol{f}, \boldsymbol{g}, \boldsymbol{h}\}$ of the Serret-Frenet system with curvature $k(x)=c e^{\alpha x^{2} / 4}$ and torsion $\tau(x)=-\beta x / 2$, and initial conditions $\boldsymbol{f}(0)=\boldsymbol{e}_{1}, \boldsymbol{g}(0)=\boldsymbol{e}_{2}$, and $\boldsymbol{h}(0)=\boldsymbol{e}_{3}$.

As done for the expanders, we provide now some properties of these solutions, that are obtained by studying the Serret-Frenet system.

Theorem 5.14 (61]). Let $\alpha \in(0,1], c>0, T \in \mathbb{R}$ and $\boldsymbol{f}_{c, \alpha}$ as above. Set $\tilde{\boldsymbol{m}}_{c, \alpha}(x, t)=$ $\boldsymbol{f}_{c, \alpha}\left(\frac{x}{\sqrt{T-t}}\right)$, for $x \in \mathbb{R}, t<T$. Then $\tilde{\boldsymbol{m}}_{c, \alpha}$ belongs to $\mathcal{C}^{\infty}\left(\mathbb{R} \times(-\infty, T) ; \mathbb{S}^{2}\right)$, solves (7) for $t \in(-\infty, T)$, and $\left|\partial_{x} \tilde{\boldsymbol{m}}_{c, \alpha}(x, t)\right|=\frac{c}{\sqrt{T-t}} e^{\frac{\alpha x^{2}}{4(T-t)}}$, for all $(x, t) \in \mathbb{R} \times(-\infty, T)$. Moreover, the following properties hold.

(i) The first component of $\boldsymbol{f}_{c, \alpha}$ is even, while the others are odd.

(ii) There exist constants $\rho_{j, c, \alpha} \in[0,1], B_{j, c, \alpha} \in[-1,1]$, and $\phi_{j, c, \alpha} \in[0,2 \pi)$, for $j \in\{1,2,3\}$, such that we have the following asymptotics for the profile $\boldsymbol{f}_{c, \alpha}$ :

$$
\begin{aligned}
f_{j, c, \alpha}(x)= & \rho_{j, c, \alpha} \cos \left(c \Phi_{\alpha}(x)-\phi_{j, c, \alpha}\right)-\frac{\beta B_{j, c, \alpha}}{2 c} x e^{-\alpha x^{2} / 4} \\
& +\frac{\beta^{2} \rho_{j, c, \alpha}}{8 c} \sin \left(c \Phi_{\alpha}(x)-\phi_{j, c, \alpha}\right) \int_{x}^{\infty} s^{2} e^{-\alpha s^{2} / 4} d s+\frac{\beta}{\alpha^{5} c^{2}} \mathcal{O}\left(x^{2} e^{-\alpha x^{2} / 2}\right),
\end{aligned}
$$

for all $x \geq 1$, where $\Phi_{\alpha}(x)=\int_{0}^{x} e^{\frac{\alpha s^{2}}{4}} d s$.

(iii) The solution $\tilde{\boldsymbol{m}}_{c, \alpha}=\left(\tilde{m}_{1, c, \alpha}, \tilde{m}_{2, c, \alpha}, \tilde{m}_{3, c, \alpha}\right)$ satisfies the following pointwise convergences

$$
\begin{aligned}
& \lim _{t \rightarrow T^{-}}\left(\tilde{m}_{j, c, \alpha}(x, t)-\rho_{j, c, \alpha} \cos \left(c \Phi_{\alpha}\left(\frac{x}{\sqrt{T-t}}\right)-\phi_{j, c, \alpha}\right)=0, \text { if } x>0,\right. \\
& \lim _{t \rightarrow T^{-}}\left(\tilde{m}_{j, c, \alpha}(x, t)-\rho_{j, c, \alpha}^{-} \cos \left(c \Phi_{\alpha}\left(\frac{-x}{\sqrt{T-t}}\right)-\phi_{j, c, \alpha}\right)=0, \text { if } x<0,\right.
\end{aligned}
$$

for $j \in\{1,2,3\}$, where $\rho_{1, c, \alpha}^{-}=\rho_{1, c, \alpha}, \rho_{2, c, \alpha}^{-}=-\rho_{2, c, \alpha}$ and $\rho_{3, c, \alpha}^{-}=-\rho_{3, c, \alpha}$.

(iv) $\tilde{\boldsymbol{m}}_{c, \alpha}(\cdot, t) \rightarrow 0$ as $t \rightarrow T^{-}$, as a tempered distribution.

As for the expanders, the big- $\mathcal{O}$ in the asymptotics does not depend on $\alpha \in[0,1]$. In this manner, the constants multiplying the big- $\mathcal{O}$ are meaningful and in particular, big- $\mathcal{O}$ vanishes when $\beta=0$. Let us remark that the behavior of the profile for $x \leq-1$ follows from the symmetries of the profile established in part (i).

In Figure 4, we have depicted the profile $\tilde{\boldsymbol{m}}_{c, \alpha}$ for $\alpha=0.5$ and $c=0.5$, where we can see the oscillating behavior. Moreover, the plots in Figure 4 suggest that the limit sets of the trajectories are great circles on the sphere $\mathbb{S}^{2}$ when $x \rightarrow \pm \infty$. This is indeed the case. The next result establishes analytically that $\tilde{\boldsymbol{m}}_{c, \alpha}$ oscillates in a plane passing through the origin whose normal vector is given by $\boldsymbol{B}_{c, \alpha}^{ \pm}=\left(B_{1, c, \alpha}, B_{2, c, \alpha}, B_{3, c, \alpha}\right)$, as $x \rightarrow \pm \infty$, respectively. 

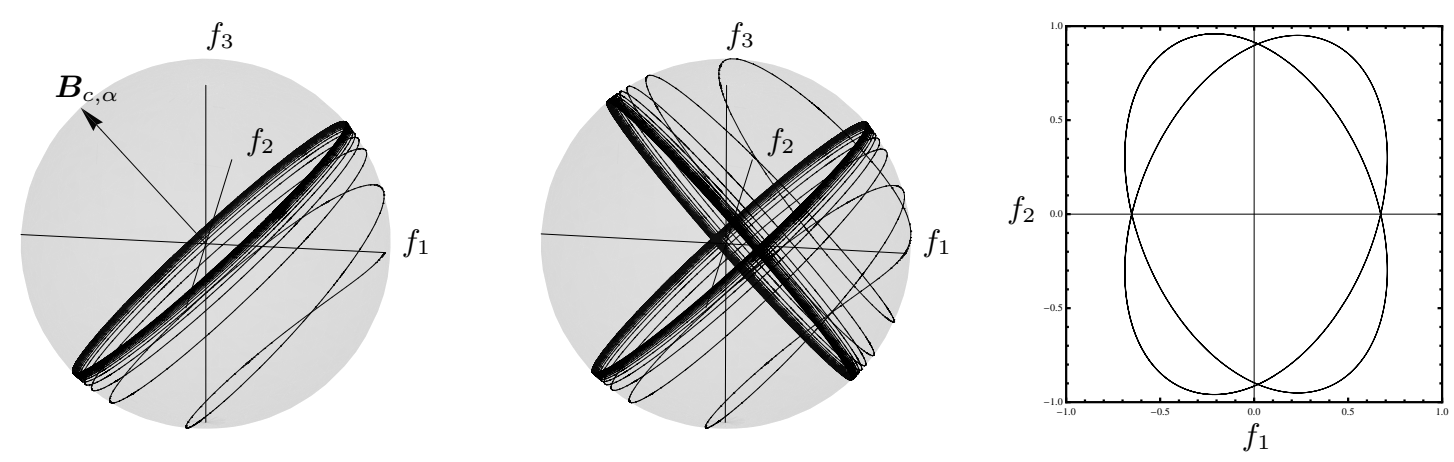

Figure 4: Profile $\boldsymbol{f}_{c, \alpha}$ for $c=0.5$ and $\alpha=0.5$. The figure on the left depicts profile for $x \in \mathbb{R}^{+}$ and the normal vector $\boldsymbol{B}_{c, \alpha} \approx(-0.72,-0.3,0.63)$. The figure on the center shows the profile for $x \in \mathbb{R}$; the angle between the circles $\mathcal{C}_{c, \alpha}^{ \pm}$is $\vartheta_{c, \alpha} \approx 1.5951$. At the right, the projection of limit cycles $\mathcal{C}_{c, \alpha}^{ \pm}$on the plane $\mathbb{R}^{2}$.

Theorem $5.15([60])$. Let $\mathcal{P}_{c, \alpha}^{ \pm}$be the planes passing through the origin with normal vectors $\boldsymbol{B}_{c, \alpha}^{ \pm}$, respectively. Let $\mathcal{C}_{c, \alpha}^{ \pm}$be the circles in $\mathbb{R}^{3}$ given by $\mathcal{C}_{c, \alpha}^{ \pm}=\mathcal{P}_{c, \alpha}^{ \pm} \cap \mathbb{S}^{2}$. Then for all $|x| \geq 1$,

$$
\left.\operatorname{dist}\left(\tilde{\boldsymbol{m}}_{c, \alpha}(x), \mathcal{C}_{c, \alpha}^{ \pm}\right)\right) \leq \frac{15 \sqrt{2} \beta}{c \alpha^{2}}|x| e^{-\alpha x^{2} / 4}
$$

In particular

$$
\left.\left.\lim _{t \rightarrow T^{-}} \operatorname{dist}\left(\tilde{\boldsymbol{m}}_{c, \alpha}(x, t), \mathcal{C}_{c, \alpha}^{+}\right)\right)=0, \text { if } x>0, \text { and } \lim _{t \rightarrow T^{-}} \operatorname{dist}\left(\tilde{\boldsymbol{m}}_{c, \alpha}(x, t), \mathcal{C}_{c, \alpha}^{-}\right)\right)=0 \text {, if } x<0 .
$$

Theorem 5.15 establishes the convergence of the profile $\boldsymbol{f}_{c, \alpha}$ to the great circles $\mathcal{C}_{c, \alpha}^{ \pm}$as shown in Figure 4 . Moreover, (49) gives us an exponential rate for this convergence. In terms of the solution $\tilde{\boldsymbol{m}}_{c, \alpha}$ to the LLG equation, this provides a more precise geometric information about the way that the solution blows up at time $T$. The existence of limit cycles for related ferromagnetic models have been investigated for instance in [101, 21], but to the best of our knowledge this is the first time that this type of phenomenon has been observed for the LLG equation. In Figure 4 one can see that $\vartheta_{c, \alpha} \approx 1.5951$ for $\alpha=0.5$ and $c=0.5$, where we have chosen the value of $c$ such that the angle is close to $\pi / 2$.

In the case $\alpha=1$, the torsion vanishes, and it easy to deduce that the profile is explicitly given by the plane curve $\boldsymbol{f}_{c, 1}(x)=\left(\cos \left(c \Phi_{1}(x)\right), \sin \left(c \Phi_{1}(x)\right), 0\right)$. In particular, we see that the asymptotics in Theorem 5.14 are satisfied with $\rho_{1, c, 1}=1, \rho_{2, c, 1}=1, \rho_{3, c, 1}=0, \phi_{1, c, 1}=0$, $\phi_{2, c, 1}=3 \pi / 2, \phi_{3, c, 1} \in[0,2 \pi)$.

In the case $\alpha=0, \boldsymbol{f}_{c, 0}$ is equal to $\mathfrak{m}_{c, 0}$ in 41 , so that $\boldsymbol{f}_{c, 0}$ converges to the point $\boldsymbol{A}_{c, 0}^{+}$, as $x \rightarrow \infty$. Hence, there is a drastic change in the behavior of the profile in the cases $\alpha=0$ and $\alpha>0$ : In the first case $\boldsymbol{f}_{c, 0}$ converges to a point at infinity, while in the second case (49) tells us that $\boldsymbol{f}_{c, \alpha}$ converges to a great circle. In this sense, there is a discontinuity in the behavior of $\tilde{\boldsymbol{m}}_{c, \alpha}$ at $\alpha=0$.

Acknowledgements. A. de Laire was partially supported by the Labex CEMPI (ANR-11LABX-0007-01), the ANR project "Dispersive and random waves" (ANR-18-CE40-0020-01), and the MATH-AmSud project EEQUADD-II. 


\section{References}

[1] F. Alouges and A. Soyeur. On global weak solutions for Landau-Lifshitz equations: existence and nonuniqueness. Nonlinear Anal., 18(11):1071-1084, 1992.

[2] Y. Bahri. Asymptotic stability in the energy space for dark solitons of the Landau-Lifshitz equation. Anal. PDE, 9(3):645-697, 2016.

[3] Y. Bahri. On the asymptotic stability in the energy space for multi-solitons of the LandauLifshitz equation. Trans. Amer. Math. Soc., 370(7):4683-4707, 2018.

[4] V. Banica and L. Vega. On the Dirac delta as initial condition for nonlinear Schrödinger equations. Ann. Inst. H. Poincaré Anal. Non Linéaire, 25(4):697-711, 2008.

[5] V. Banica and L. Vega. On the stability of a singular vortex dynamics. Comm. Math. Phys., 286(2):593-627, 2009.

[6] V. Banica and L. Vega. Scattering for 1D cubic NLS and singular vortex dynamics. J. Eur. Math. Soc. (JEMS), 14(1):209-253, 2012.

[7] V. Banica and L. Vega. Stability of the self-similar dynamics of a vortex filament. Arch. Ration. Mech. Anal., 210(3):673-712, 2013.

[8] V. Banica and L. Vega. Singularity formation for the 1-D cubic NLS and the Schrödinger map on $\mathbb{S}^{2}$. Commun. Pure Appl. Anal., 17(4):1317-1329, 2018.

[9] I. Bejenaru, A. Ionescu, C. Kenig, and D. Tataru. Global Schrödinger maps in dimensions $d \geq 2$ : Small data in the critical Sobolev spaces. Annals of Math., 173(3):1443-1506, 2011.

[10] F. Béthuel, R. Danchin, and D. Smets. On the linear wave regime of the Gross-Pitaevskii equation. J. Anal. Math., 110(1):297-338, 2009.

[11] F. Béthuel, P. Gravejat, and J.-C. Saut. Existence and properties of travelling waves for the Gross-Pitaevskii equation. In A. Farina and J.-C. Saut, editors, Stationary and time dependent Gross-Pitaevskii equations, volume 473 of Contemp. Math., pages 55-104. Amer. Math. Soc., Providence, RI, 2008.

[12] F. Béthuel, P. Gravejat, J.-C. Saut, and D. Smets. Orbital stability of the black soliton for the Gross-Pitaevskii equation. Indiana Univ. Math. J, 57(6):2611-2642, 2008.

[13] F. Béthuel, P. Gravejat, J.-C. Saut, and D. Smets. On the Korteweg-de Vries long-wave approximation of the Gross-Pitaevskii equation II. Commun. Partial Differential Equations, $35(1): 113-164,2010$.

[14] F. Béthuel, P. Gravejat, and D. Smets. Stability in the energy space for chains of solitons of the one-dimensional Gross-Pitaevskii equation. Ann. Inst. Fourier, 64(1), 2014.

[15] P. Biernat and P. Bizoń. Shrikers, expanders, and the unique continuation beyond generic blowup in the heat flow for harmonic maps between spheres. Nonlinearity, 24(8):2211-2228, 2011.

[16] P. Biernat and R. Donninger. Construction of a spectrally stable self-similar blowup solution to the supercritical corotational harmonic map heat flow. Nonlinearity, 31(8):3543, 2018. 
[17] R. Bikbaev, A. Bobenko, and A. Its. Landau-Lifshitz equation, uniaxial anisotropy case: Theory of exact solutions. Theoret. and Math. Phys., 178(2):143-193, 2014.

[18] A. Bishop and K. Long. Nonlinear excitations in classical ferromagnetic chains. J. Phys. A, 12(8):1325-1339, 1979.

[19] P. Bizoń and A. Wasserman. Nonexistence of shrinkers for the harmonic map flow in higher dimensions. Int. Math. Res. Not. IMRN, (17):7757-7762, 2015.

[20] D. Bresch, M. Gisclon, and I. Lacroix-Violet. On Navier-Stokes-Korteweg and EulerKorteweg Systems: Application to Quantum Fluids Models. Arch. Ration. Mech. Anal., 233(3):975-1025, 2019.

[21] G. Broggi, P. F. Meier, R. Stoop, and R. Badii. Nonlinear dynamics of a model for parallel pumping in ferromagnets. Phys. Rev. A, 35:365-368, Jan 1987.

[22] R. Buckingham and P. Miller. Exact solutions of semiclassical non-characteristic Cauchy problems for the Sine-Gordon equation. Phys. D, 237(18):2296-2341, 2008.

[23] R. Carles, R. Danchin, and J.-C. Saut. Madelung, Gross-Pitaevskii and Korteweg. Nonlinearity, 25(10):2843-2873, 2012.

[24] T. Cazenave. Semilinear Schrödinger equations, volume 10 of Courant Lecture Notes in Mathematics. Amer. Math. Soc., Providence, 2003.

[25] N.-H. Chang, J. Shatah, and K. Uhlenbeck. Schrödinger maps. Comm. Pure Appl. Math., $53(5): 590-602,2000$.

[26] D. Chiron. Error bounds for the (KdV)/(KP-I) and the (gKdV)/(gKP-I) asymptotic regime for nonlinear Schrödinger type equations. Ann. Inst. Henri Poincaré, Analyse Non Linéaire, 31(6):1175-1230, 2014.

[27] D. Chiron and F. Rousset. The KdV/KP-I limit of the nonlinear Schrödinger equation. SIAM J. Math. Anal., 42(1):64-96, 2010.

[28] V. Chousionis, M. B. Erdogan, and N. Tzirakis. Fractal solutions of linear and nonlinear dispersive partial differential equations. Proc. Lond. Math. Soc. (3), 110(3):543-564, 2015.

[29] I. Cimrák. A survey on the numerics and computations for the Landau-Lifshitz equation of micromagnetism. Arch. Comput. Methods Eng., 15(3):277-309, 2008.

[30] J.-M. Coron. Nonuniqueness for the heat flow of harmonic maps. Ann. Inst. H. Poincaré Anal. Non Linéaire, 7(4):335-344, 1990.

[31] M. Daniel and M. Lakshmanan. Perturbation of solitons in the classical continuum isotropic Heisenberg spin system. Physica A: Statistical Mechanics and its Applications, 120(1):125-152, 1983.

[32] A. de Laire. Global well-posedness for a nonlocal Gross-Pitaevskii equation with non-zero condition at infinity. Commun. Partial Differential Equations, 35(11):2021-2058, 2010.

[33] A. de Laire. Minimal energy for the traveling waves of the Landau-Lifshitz equation. SIAM J. Math. Anal., 46(1):96-132, 2014.

[34] A. de Laire and P. Gravejat. Stability in the energy space for chains of solitons of the Landau-Lifshitz equation. J. Differential Equations, 258(1):1-80, 2015. 
[35] A. de Laire and P. Gravejat. The Sine-Gordon regime of the Landau-Lifshitz equation with a strong easy-plane anisotropy. Ann. Inst. Henri Poincaré, Analyse Non Linéaire, 35(7):1885-1945, 2018.

[36] A. de Laire and P. Gravejat. The cubic Schrödinger regime of the Landau-Lifshitz equation with a strong easy-axis anisotropy. Rev. Mat. Iberoam., 37(1):95-128, 2021.

[37] A. de Laire and P. Mennuni. Traveling waves for some nonlocal 1D Gross-Pitaevskii equations with nonzero conditions at infinity. Discrete Contin. Dyn. Syst., 40(1):635-682, 2020 .

[38] A. Deruelle and T. Lamm. Existence of expanders of the harmonic map flow. Preprint arXiv:1801.08012.

[39] S. Ding and C. Wang. Finite time singularity of the Landau-Lifshitz-Gilbert equation. Int. Math. Res. Not. IMRN, (4):Art. ID rnm012, 25, 2007.

[40] W. Ding and Y. Wang. Schrödinger flow of maps into symplectic manifolds. Sci. China Ser. A, 41(7):746-755, 1998.

[41] J. Eells and L. Lemaire. Another report on harmonic maps. Bull. London Math. Soc., 20(5):385-524, 1988.

[42] J. Eggers and M. A. Fontelos. The role of self-similarity in singularities of partial differential equations. Nonlinearity, 22(1):1-9, 2009.

[43] L. Faddeev and L. Takhtajan. Hamiltonian methods in the theory of solitons. Classics in Mathematics. Springer-Verlag, Berlin-Heidelberg-New York, 2007. Translated by A.G. Reyman.

[44] H. Fan. Existence of the self-similar solutions in the heat flow of harmonic maps. Sci. China Ser. A, 42(2):113-132, 1999.

[45] O. Gamayun and O. Lisovyy. On self-similar solutions of the vortex filament equation. $J$. Math. Phys., 60(8):083510, 13, 2019.

[46] A. Gastel. Singularities of first kind in the harmonic map and Yang-Mills heat flows. Math. Z., 242(1):47-62, 2002.

[47] P. Gérard and Z. Zhang. Orbital stability of traveling waves for the one-dimensional Gross-Pitaevskii equation. J. Math. Pures Appl., 91(2):178-210, 2009.

[48] P. Germain, T.-E. Ghoul, and H. Miura. On uniqueness for the harmonic map heat flow in supercritical dimensions. Comm. Pure Appl. Math., 70(12):2247-2299, 2017.

[49] P. Germain and F. Rousset. Long wave limit for Schrödinger maps. J. Eur. Math. Soc., 21(8):2517-2602, 2019.

[50] P. Germain and M. Rupflin. Selfsimilar expanders of the harmonic map flow. Ann. Inst. H. Poincaré Anal. Non Linéaire, 28(5):743-773, 2011.

[51] P. Germain, J. Shatah, and C. Zeng. Self-similar solutions for the Schrödinger map equation. Math. Z., 264(3):697-707, 2010.

[52] M.-H. Giga, Y. Giga, and J. Saal. Nonlinear partial differential equations, volume 79 of Progress in Nonlinear Differential Equations and their Applications. Birkhäuser Boston, Inc., Boston, MA, 2010. Asymptotic behavior of solutions and self-similar solutions. 
[53] T. L. Gilbert. A lagrangian formulation of the gyromagnetic equation of the magnetization field. Phys. Rev., 100:1243, 1955.

[54] M. Grillakis, J. Shatah, and W. Strauss. Stability theory of solitary waves in the presence of symmetry I. J. Funct. Anal., 74(1):160-197, 1987.

[55] A. Grünrock. Bi- and trilinear Schrödinger estimates in one space dimension with applications to cubic NLS and DNLS. Int. Math. Res. Not., (41):2525-2558, 2005.

[56] M. Guan, S. Gustafson, K. Kang, and T.-P. Tsai. Global questions for map evolution equations. In Singularities in PDE and the calculus of variations, volume 44 of CRM Proc. Lecture Notes, pages 61-74. Amer. Math. Soc., Providence, RI, 2008.

[57] B. Guo and S. Ding. Landau-Lifshitz equations, volume 1 of Frontiers of Research with the Chinese Academy of Sciences. World Scientific, Hackensack, 2008.

[58] S. Gustafson and J. Shatah. The stability of localized solutions of Landau-Lifshitz equations. Comm. Pure Appl. Math., 55(9):1136-1159, 2002.

[59] S. Gutiérrez and A. de Laire. Self-similar solutions of the one-dimensional Landau-LifshitzGilbert equation. Nonlinearity, 28(5):1307-1350, 2015.

[60] S. Gutiérrez and A. de Laire. The Cauchy problem for the Landau-Lifshitz-Gilbert equation in BMO and self-similar solutions. Nonlinearity, 32(7):2522-2563, 2019.

[61] S. Gutiérrez and A. de Laire. Self-similar shrinkers of the one-dimensional Landau-LifshitzGilbert equation. J. Evol. Equ., 21(1):473-501, 2021.

[62] S. Gutiérrez, J. Rivas, and L. Vega. Formation of singularities and self-similar vortex motion under the localized induction approximation. Comm. Partial Differential Equations, 28(5-6):927-968, 2003.

[63] S. Gutiérrez and L. Vega. Self-similar solutions of the localized induction approximation: singularity formation. Nonlinearity, 17:2091-2136, 2004.

[64] H. Hasimoto. A soliton on a vortex filament. J. Fluid Mech, 51:477-485, 1972.

[65] R. Jerrard and D. Smets. On Schrödinger maps from $\mathbb{T}^{1}$ to $\mathbb{S}^{2}$. Ann. Sci. Éc. Norm. Sup., 45(4):635-678, 2012.

[66] H. Jia, V. Sverak, and T.-P. Tsai. Self-similar solutions to the nonstationary navier-stokes equations. In Handbook of Mathematical Analysis in Mechanics of Viscous Fluids, pages 461-507. Springer, 2018.

[67] C. E. Kenig, G. Ponce, and L. Vega. On the ill-posedness of some canonical dispersive equations. Duke Math. J., 106(3):617-633, 2001.

[68] H. Koch and T. Lamm. Geometric flows with rough initial data. Asian J. Math., 16(2):209$235,2012$.

[69] H. Koch and D. Tataru. Well-posedness for the Navier-Stokes equations. Adv. Math., $157(1): 22-35,2001$.

[70] R. Kohn, A. DeSimone, F. Otto, and S. Mueller. Recent analytical developments in micromagnetics. In G. Bertotti and I. D. Mayergoyz, editors, The science of hysteresis. Vol. II: Physical modeling, micromagnetics, and magnetization dynamics, pages 269-381. Elsevier, 2006. 
[71] M. Lakshmanan. The fascinating world of the Landau-Lifshitz-Gilbert equation: an overview. Philos. Trans. R. Soc. Lond. Ser. A Math. Phys. Eng. Sci., 369(1939):1280-1300, 2011.

[72] M. Lakshmanan and K. Nakamura. Landau-Lifshitz equation of ferromagnetism: Exact treatment of the Gilbert damping. Phys. Rev. Lett., 53:2497-2499, 1984.

[73] M. Lakshmanan, T. Ruijgrok, and C. Thompson. On the dynamics of a continuum spin system. Phys. A, 84(3):577-590, 1976.

[74] M. Lakshmanan, T. W. Ruijgrok, and C. Thompson. On the dynamics of a continuum spin system. Physica A: Statistical Mechanics and its Applications, 84(3):577-590, 1976.

[75] G. L. Lamb, Jr. Elements of soliton theory. John Wiley \& Sons Inc., New York, 1980. Pure and Applied Mathematics, A Wiley-Interscience Publication.

[76] L. Landau and E. Lifshitz. On the theory of the dispersion of magnetic permeability in ferromagnetic bodies. Phys. Zeitsch. der Sow., 8:153-169, 1935.

[77] P. G. Lemarié-Rieusset. Recent developments in the Navier-Stokes problem, volume 431 of Chapman $\&$ Hall/CRC Research Notes in Mathematics. Chapman \& Hall/CRC, Boca Raton, FL, 2002.

[78] F. Lin and C. Wang. The analysis of harmonic maps and their heat flows. World Scientific Publishing Co. Pte. Ltd., Hackensack, NJ, 2008.

[79] J. Lin, B. Lai, and C. Wang. Global well-posedness of the Landau-Lifshitz-Gilbert equation for initial data in Morrey spaces. Calc. Var. Partial Differential Equations, 54(1):665-692, 2015 .

[80] E. Madelung. Quantumtheorie in Hydrodynamische form. Zts. f. Phys., 40:322-326, 1926.

[81] Y. Martel and F. Merle. Asymptotic stability of solitons of the subcritical gKdV equations revisited. Nonlinearity, 18(1):55-80, 2005.

[82] Y. Martel and F. Merle. Asymptotic stability of solitons of the gKdV equations with general nonlinearity. Math. Ann., 341(2):391-427, 2008.

[83] Y. Martel and F. Merle. Stability of two soliton collision for nonintegrable gKdV equations. Comm. Math. Phys., 286(1):39-79, 2009.

[84] Y. Martel and F. Merle. Inelastic interaction of nearly equal solitons for the quartic gKdV equation. Invent. Math., 183(3):563-648, 2011.

[85] Y. Martel, F. Merle, and T.-P. Tsai. Stability and asymptotic stability in the energy space of the sum of $N$ solitons for subcritical gKdV equations. Comm. Math. Phys., 231(2):347-373, 2002.

[86] Y. Martel, F. Merle, and T.-P. Tsai. Stability in $H^{1}$ of the sum of $K$ solitary waves for some nonlinear Schrödinger equations. Duke Math. J., 133(3):405-466, 2006.

[87] H. McGahagan. An approximation scheme for Schrödinger maps. Commun. Partial Differential Equations, 32(3):375-400, 2007.

[88] C. Melcher. Global solvability of the Cauchy problem for the Landau-Lifshitz-Gilbert equation in higher dimensions. Indiana Univ. Math. J., 61(3):1175-1200, 2012. 
[89] J. Moser. A rapidly convergent iteration method and non-linear differential equations. II. Ann. Scuola Norm. Sup. Pisa (3), 20(3):499-535, 1966.

[90] A. Nahmod, J. Shatah, L. Vega, and C. Zeng. Schrödinger maps and their associated frame systems. Int. Math. Res. Not., 2007:1-29, 2007.

[91] I. Rodnianski, Y. A. Rubinstein, and G. Staffilani. On the global well-posedness of the one-dimensional Schrödinger map flow. Anal. PDE, 2(2):187-209, 2009.

[92] J. Shatah and C. Zeng. Schrödinger maps and anti-ferromagnetic chains. Comm. Math. Phys., 262(2):299-315, 2006.

[93] E. Sklyanin. On complete integrability of the Landau-Lifshitz equation. Technical Report E-3-79, Leningrad Department of Steklov Institute of Mathematics of the USSR Academy of Sciences, 1979.

[94] C. Song and Y. Wang. Uniqueness of Schrödinger flow on manifolds. Comm. Anal. Geom., 26(1):217-235, 2018.

[95] E. M. Stein. Harmonic analysis: real-variable methods, orthogonality, and oscillatory integrals, volume 43 of Princeton Mathematical Series. Princeton University Press, Princeton, NJ, 1993. With the assistance of Timothy S. Murphy, Monographs in Harmonic Analysis, III.

[96] M. Struwe. Geometric evolution problems. In Nonlinear partial differential equations in differential geometry (Park City, UT, 1992), volume 2 of IAS/Park City Math. Ser., pages 257-339. Amer. Math. Soc., Providence, RI, 1996.

[97] P.-L. Sulem, C. Sulem, and C. Bardos. On the continuous limit for a system of classical spins. Comm. Math. Phys., 107(3):431-454, 1986.

[98] M. Taylor. Partial differential equations III, volume 117 of Applied Mathematical Sciences. Springer-Verlag, New-York, Second edition, 2011.

[99] J. Tjon and J. Wright. Solitons in the continuous Heisenberg spin chain. Phys. Rev. B, 15(7):3470-3476, 1977.

[100] A. Vargas and L. Vega. Global wellposedness for 1D non-linear Schrödinger equation for data with an infinite $L^{2}$ norm. J. Math. Pures Appl. (9), 80(10):1029-1044, 2001.

[101] F. Waldner, D. R. Barberis, and H. Yamazaki. Route to chaos by irregular periods: Simulations of parallel pumping in ferromagnets. Phys. Rev. A, 31:420-431, 1985.

[102] C. Wang. Well-posedness for the heat flow of harmonic maps and the liquid crystal flow with rough initial data. Arch. Ration. Mech. Anal., 200(1):1-19, 2011.

[103] D. Wei. Micromagnetics and Recording Materials. SpringerBriefs in Applied Sciences and Technology. Springer Berlin Heidelberg, 2012.

[104] Y. Zhou and B. Guo. Existence of weak solution for boundary problems of systems of ferro-magnetic chain. Sci. China Ser. A, 27(8):799-811, 1984. 